\title{
Interpolation Theorem and Characterization Theorem
}

\author{
Nobuyoshi MotohashI \\ Gakushuin University, Tokyo
}

\section{Introduction}

In this paper, we shall show some examples of the applications of "normal derivation theorem" in Motohashi [27] to a new kind of problems named "interpolation problem and characterization problem" which can be considered as a generalized treatment of Craig-Lyndon-like interpolation theorems (cf. Craig [2], [3], Lyndon [17]), relativization theorem (cf. Robinson [31]) and preservation theorems in model theory (cf. Keisler [9], Lyndon [16], Shoenfield [33], Robinson [31]).

As an explanation of what lies behind our problems, let us remind ourselves of well-known interpolation theorems, relativization theorem and some preservation theorems in the first order logic.

Let $\mathscr{L}$ be a first order logic with $P C$ as the set of predicate constants and an equality symbol $E$.

Let $L$ be another first order logic whose predicate constants are $P^{\mathbf{1}}, P^{\mathbf{2}}$ for $P \in P C$, one unary predicate constant $U$ and two binary predicate constants $I_{0}$, $I_{1}$.

We assume that $P^{1}, P^{2}(P \in P C), U, I_{0}, I_{1}$, are all distinct. In $\mathscr{L}$ we assume the equality axioms for $E$ with respect to $P \in P C$ but in $L$, we only assume the axioms saying that $E^{1}$ has the same role as the equality symbol with respect to $P^{1}$ for $P \in P C$ and $E^{2}$ has the same role as the equality symbol with respect to $P^{2}$ for $P \in P C$. Then an $\mathscr{L}$-structure $\mathscr{A}$ is a mapping from $P C$ to the set of all finitary relations on a non empty set $|\mathscr{A}|$ (called the universe of $\mathscr{A}$ ) such that $\mathscr{A}(P) \subseteq|\mathscr{A}|^{k}$ for any $k$-ary $P \in P C$ and $\mathscr{A}(E)$ is the identity relation on $|\mathscr{A}|$. But an $L$-structure $M$ is not an usual first order structure. $M$ is a mapping from $\left\{P^{1}, P^{2} ; P \in P C\right\}^{\cup}\left\{U, I_{0}, I_{1}\right\}$ to the set of finitary relations on a non empty set $|M|$ (called the universe of $M$ ) such that $M\left(P^{1}\right), M\left(P^{2}\right) \subseteq|M|^{k}$ for any $k$-ary $P \in P C, M(U) \subseteq|M|, M\left(I_{0}\right), M\left(I_{1}\right) \subseteq|M|^{2}$, $M\left(E^{1}\right)$ is a congruence relation with respect to $M\left(P^{1}\right)$ for $P \in P C$ and $M\left(E^{2}\right)$ is a congruence relation with respect to $M\left(P^{2}\right)$ for $P \in P C$.

Hence, through factorization by $M\left(E^{1}\right), M\left(E^{2}\right)$, we get two $\mathscr{L}$-structures $M_{1}, M_{2}$ by $\left|M_{1}\right|=|M| / M\left(E^{1}\right), \quad\left|M_{2}\right|=|M| / M\left(E^{2}\right), \quad M_{1}(P)=M\left(P^{1}\right) / M\left(E^{1}\right), \quad M_{2}(P)=$ $M\left(P^{2}\right) / M\left(E^{2}\right)$ for $P \in P C$.

Let

$$
\begin{gathered}
M^{*}(U)=M(U) / M\left(E^{1}\right)=\left\{m^{* 1} ; m \in M(U)\right\}, \\
-85-
\end{gathered}
$$




$$
\begin{aligned}
& M^{*}\left(I_{0}\right)=\left\{\left\langle m^{* 1}, n^{* 2}\right\rangle ;\langle m, n\rangle \in M\left(I_{0}\right)\right\}, \\
& M^{*}\left(I_{1}\right)=\left\{\left\langle m^{* 1}, n^{* 2}\right\rangle ;\langle m, n\rangle \in M\left(I_{1}\right)\right\},
\end{aligned}
$$

where $m^{* 1}$ is the equivalence class of $m$ by $M\left(E^{1}\right)$ and $n^{* 2}$ is the equivalence class of $n$ by $M\left(E^{2}\right)$.

Then $M^{*}(U)$ is a subset of $\left|M_{1}\right|, M^{*}\left(I_{0}\right), M^{*}\left(I_{1}\right)$ are relations between $\left|M_{1}\right|$ and $\left|M_{2}\right|$ (i.e. $\left.M^{*}\left(I_{0}\right), M^{*}\left(I_{1}\right) \subseteq\left|M_{1}\right| \times\left|M_{2}\right|\right)$.

So, every sentence in $L$ can be considered as expressing a relation between $\mathscr{L}$ structures as is shown in the followng examples.

For each formula $\theta$ in $\mathscr{L}$, let $\theta^{1}$ be the formula in $L$ obtained from $\theta$ by replacing every $P \in P C$ by $P^{1}$ and $\theta^{2}$ by replacing $P$ by $P^{2}$.

Then for any formula $\theta\left(x_{1}, \cdots, x_{n}\right)$ in $\mathscr{L}$ whose free variables are among $x_{1}, \cdots$, $x_{n}$, any $L$-structure $M$ and any elements $m_{1}, \cdots, m_{n}$ in $|M|$,

$$
\begin{aligned}
& M \models \theta^{1}\left[m_{1}, \cdots, m_{n}\right] \Leftrightarrow M_{1} \models \theta\left[m_{1}^{* 1}, \cdots, m_{n}^{* 1}\right] \\
& M \vDash \theta^{2}\left[m_{1}, \cdots, m_{n}\right] \Leftrightarrow M_{2} \models \theta\left[m_{1}^{* 2}, \cdots, m_{n}^{* 2}\right] .
\end{aligned}
$$

Let $Q$ be an unary predicate in $P C$.

\section{EXAMPLE 1}

Let $\Psi_{1}=\left\{(\forall u)\left(Q^{1}(u) \supset Q^{2}(u)\right),(\forall u)\left(\neg Q^{1}(u) \supset \neg Q^{2}(u)\right)\right.$,

$$
\left.(\forall u)(\forall v)\left(E^{1}(u, v) \supset E^{2}(u, v)\right),(\forall u)(\forall v)\left(\neg E^{1}(u, v) \supset \neg E^{2}(u, v)\right)\right\} .
$$

Then

$M \models \Psi_{1}$ expresses that $\left|M_{1}\right|=\left|M_{2}\right|$ and $M_{1}(Q)=M_{2}(Q)$. (Of course $M \models \Psi_{1}$ means $M \models \varphi$ for any $\left.\varphi \in \Psi_{1}\right)$.

EXAMPLE 2

Let $\Psi_{2}=\left\{(\forall u)(\exists v) I_{0}(u, v),(\forall v)(\exists u) I_{0}(u, v)\right.$,

$$
(\forall u)(\forall v)\left(I_{0}(u, v) \wedge Q^{1}(u) . \supset Q^{2}(v)\right),
$$$$
(\forall u)(\forall v)\left(I_{0}(u, v)_{\frown} \rightarrow Q^{1}(u) . \supset \neg Q^{2}(v)\right),
$$$$
\left(\forall u_{1}\right)\left(\forall u_{2}\right)\left(\forall v_{1}\right)\left(\forall v_{2}\right)\left(I_{0}\left(u_{1}, v_{1}\right) \curvearrowright I_{0}\left(u_{2}, v_{2}\right)_{\curvearrowright} E^{1}\left(u_{1}, u_{2}\right) . \supset E^{2}\left(v_{1}, v_{2}\right)\right) \text {, }
$$$$
\left.\left(\forall u_{1}\right)\left(\forall u_{2}\right)\left(\forall v_{1}\right)\left(\forall v_{2}\right)\left(I_{0}\left(u_{1}, v_{1}\right) \frown I_{0}\left(u_{2}, v_{2}\right)_{\curlywedge} \neg E\left(u_{1}, u_{2}\right) . \supset \neg E^{2}\left(v_{1}, v_{2}\right)\right)\right\} .
$$

Then $M \models \Psi_{2}$ expresses that $M^{*}\left(I_{0}\right)$ is a bijection from $\left|M_{1}\right|$ to $\left|M_{2}\right|$ preserving $Q$.

Let $P, R$ be two unary predicates in $P C$.

\section{EXAMPLe 3}

Let $\Psi_{3}=\Psi_{1} \cup\left\{(\forall u)\left(P^{1}(u) \supset P^{2}(u)\right),(\forall u)\left(\neg R^{1}(u) \supset \neg R^{2}(u)\right)\right\}$.

Then $M \models \Psi_{3}$ expresses that $\left|M_{1}\right|=\left|M_{2}\right|, \quad M_{1}(Q)=M_{2}(Q), \quad M_{1}(P) \subseteq M_{2}(P)$, $M_{1}(R) \supseteq M_{2}(R)$. 


\section{EXAMPLE 4}

Let $\Psi_{4}=\Psi_{2} \cup\left\{(\forall u)(\forall v)\left(I_{0}(u, v)_{\text {人 }} P^{1}(u) . \supset P^{2}(v)\right)\right.$,

$$
\left.(\forall u)(\forall v)\left(I_{0}(u, v)_{\frown} \neg R^{1}(u) . \supset \neg R^{2}(v)\right)\right) .
$$

Then $M \models \Psi_{4}$ expresses that $M^{*}\left(I_{0}\right)$ is a bijection from $\left|M_{1}\right|$ to $\left|M_{2}\right|$ such that

$$
\begin{aligned}
& M^{*}\left(I_{0}\right)\left(M_{1}(Q)\right)=M_{2}(Q), \\
& M^{*}\left(I_{0}\right)\left(M_{1}(P)\right) \subseteq M_{2}(P), \\
& M^{*}\left(I_{0}\right)\left(M_{1}(R)\right) \supseteq M_{2}(R) .
\end{aligned}
$$

EXAMPLE 5

Let $\Psi_{5}=\left\{(\exists u) U(u),(\forall \vec{u})\left(U(\vec{u}) \wedge P^{1}(\vec{u}) . \supset P^{2}(\vec{u})\right)\right.$,

$$
\left.(\forall \vec{u})\left(U(\vec{u})_{\wedge} \neg P^{1}(\vec{u}) . \supset \neg P^{2}(\vec{u})\right) ; P \in P C\right\} .
$$

$\left(U(\vec{u})\right.$ means $\left.U\left(u_{1}\right) \ldots \ldots, U\left(u_{n}\right)\right)$.

Then $M \models \Psi_{5}$ expresses that $M_{1} \mid M^{*}(U)$ is a substructure of $M_{2}$, where $M_{1} \mid M^{*}(U)$ is the restriction of $M_{1}$ to $M^{*}(U)$ with the identication of $m^{* 1}$ by $m^{* 2}$ for each $m \in M(U)$.

EXAMPLE 6

$$
\text { Let } \begin{aligned}
\Psi_{6}= & \left\{(\forall u)(\exists v) I_{0}(u, v),\right. \\
& (\forall \vec{u})(\forall \vec{v})\left(I_{0}(\vec{u}, \vec{v}) \wedge P^{1}(\vec{u}) . \supset P^{2}(\vec{v})\right), \\
& \left.(\forall \vec{u})(\forall \vec{v})\left(I_{0}(\vec{u}, \vec{v}) \wedge \neg P^{1}(\vec{u}) . \supset \neg P^{2}(\vec{v})\right) ; P \in P C\right\},
\end{aligned}
$$

where $I_{0}(\vec{u}, \vec{v})$ means $I_{0}\left(u_{1}, v_{1}\right), \cdots, I_{0}\left(u_{n}, v_{n}\right)$.

Then $M \models \Psi_{6}$ expresses that $M^{*}\left(I_{0}\right)$ is an embedding of $M_{1}$ to $M_{2}$.

Let $<$ be a fixed binary predicate in $P C$.

\section{EXAMPLE 7}

Let $\Psi_{7}=\Psi_{5}^{\mathrm{U}}\left\{\left(\forall u_{1}\right)\left(\forall v_{1}\right)\left(\exists u_{2}\right)\left(U\left(v_{1}\right) \wedge u_{1}<{ }^{2} v_{1} . \supset . U\left(u_{2}\right) \wedge E^{2}\left(u_{1}, u_{2}\right)\right)\right\}$.

Then $M=\Psi_{7}$ expresses that $M_{2}$ is an end-extension of $M_{1} \mid M^{*}(U)$.

\section{EXAMPLE 8}

Let $\Psi_{8}=\Psi_{6} \cup\left\{\left(\forall u_{1}\right)\left(\forall v_{1}\right)\left(\forall v_{2}\right)\left(\exists u_{2}\right)\left(v_{2}<^{2} v_{1 \frown} I_{0}\left(u_{1}, v_{1}\right) \cdot \supset I_{0}\left(u_{2}, v_{2}\right)\right)\right\}$.

Then $M \models \Psi_{\mathrm{B}}$ expresses that $M_{2}$ is an end-extension of the image of $M_{1}$ by $M *\left(I_{0}\right)$.

\section{Example 9}

Let $\quad \Psi_{9}=\left\{(\forall u)(\exists v) I_{0}(u, v),(\forall v)(\exists u) I_{0}(u, v)\right.$,

$$
\left.(\forall \vec{u})(\forall \vec{v})\left(I_{0}(\vec{u}, \vec{v})_{\wedge} P^{1}(\vec{u}) . \supset P^{2}(\vec{v})\right) ; P \in P C\right\} .
$$


Then $M \models \Psi_{9}$ expresses that $M^{*}\left(I_{0}\right)$ is a homomorphism of $M_{1}$ to $M_{2}$.

\section{Example 10}

Let $\Psi_{10}=\Psi_{9}^{U}\left\{(\forall v)(\exists u) I_{1}(u, v)\right.$,

$$
\begin{aligned}
& (\forall \vec{u})(\forall \vec{v})\left(I_{1}(\vec{u}, \vec{v}), P^{1}(\vec{u}) . \supset P^{2}(\vec{v})\right) ， \\
& \left.(\forall \vec{u})(\forall \vec{v})\left(I_{1}(\vec{u}, \vec{v})_{\curlywedge} \rightarrow P^{1}(\vec{u}) . \supset \neg P^{2}(\vec{v})\right) ; P \in P C\right\}
\end{aligned}
$$

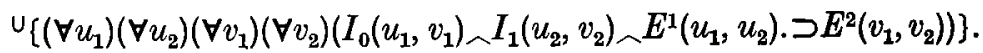

Then $M \models=\Psi_{10}$ expresses that $M^{*}\left(I_{0}\right)$ is a retract from $M_{1}$ to $M_{2}$,

i.e. $M^{*}\left(I_{0}\right)$ is a homomorphism of $M_{1}$ to $M_{2}$, $M^{*}\left(I_{1}\right)$ is an embedding of $M_{2}$ to $M_{1}$, $M^{*}\left(I_{0}\right)_{0} M^{*}\left(I_{1}\right)$ is the identity on $\left|M_{2}\right|$, (where $\left(M^{*}\left(I_{0}\right) \circ M^{*}\left(I_{1}\right)\right)(m)=\left(M^{*}\left(I_{0}\right)\right)\left(M^{*}\left(I_{1}\right)(m)\right)$.)

By using these examples $\Psi_{1}, \cdots, \Psi_{10}$ we can express many known results.

Let $X, Y$ be two mutually disjoint infinite sets of free variables in $\mathscr{L}$.

(1) Craig's interpolation theorem

Suppose $\varphi, \psi$ are sentences (i.e. closed formulas) in $\mathscr{L}$ whose common predicates are $Q, E$ only.

Then

$$
\begin{aligned}
& \vdash_{\mathscr{L}} \boldsymbol{\varphi} \supset \psi \\
& \Leftrightarrow \\
& \vdash_{\mathscr{L}} \boldsymbol{\varphi} \supset \sigma \text { and } \vdash_{\mathscr{L}} \boldsymbol{\sigma} \supset \text { for some sentence } \sigma \text { in } \mathscr{L} \text { whose predicates are } \\
& Q, E \text { only. }
\end{aligned}
$$

By using $L$, we can restate this fact in the two fashions as follow:

$$
\vdash_{\mathscr{L}} \boldsymbol{\varphi} \supset \psi
$$

$\stackrel{\leftrightarrow}{(1)}$

$$
\Psi_{1} \vdash_{L} \varphi^{1} \supset \psi^{2}
$$

(2)

$$
\vdash_{\mathscr{L}} \varphi \supset \sigma \text { and } \vdash_{\mathscr{L}} \sigma \supset \psi \text { for some sentence } \sigma \in \Delta_{1} \text {, }
$$

where $\Delta_{1}$ is the least set satisfying

(a) $Q(x), \neg Q(x), E(x, y), \neg E(x, y) \in \Delta_{1} \quad$ for any free variables $x, y$,

(b) $\Delta_{1}$ is closed under $\wedge, \vee, \forall, \exists$,

or $\vdash_{\mathscr{L}} \boldsymbol{\varphi} \supset \psi$

$\stackrel{\Leftrightarrow}{(3)}$

$$
\Psi_{2} \vdash_{L} \boldsymbol{\varphi}^{1} \supset \psi^{2}
$$


$\stackrel{\leftrightarrow}{(4)}$

$$
\vdash_{\mathscr{L}} \varphi \supset \sigma \text { and } \vdash_{\mathscr{L}} \sigma \supset \psi \quad \text { for some sentence } \sigma \in \Delta_{2} \text {, }
$$

where $\Delta_{2}$ is the least set satisfying

(a) $Q\left(x_{1}\right), \neg Q\left(x_{1}\right), E\left(x_{1}, x_{2}\right), \neg E\left(x_{1}, x_{2}\right) \in \Delta_{2}$ for any $x_{1}, x_{2} \in X$,

(b) $\Delta_{2}$ is closed under $\wedge, \vee$,

(C) $\Delta_{2}$ is closed under $\forall$,

(d) $\Delta_{2}$ is closed under $\exists$.

(2) Lyndon's interpolation theorem

Suppose $\varphi, \psi$ are sentences in $\mathscr{L}$ whose common predicates are $P, Q, R, E$ and $P$ has only positive occurrences in $\varphi, R$ has only negative occurences in $\psi$.

Then

$$
\vdash_{\mathscr{L}} \boldsymbol{\varphi} \supset \psi
$$

$\Leftrightarrow$

$\vdash_{\mathscr{L}} \boldsymbol{\varphi} \supset \sigma$ and $\vdash_{\mathscr{L}} \sigma \supset \psi$ for some sentence $\sigma$ whose predicates are $P, Q, E, R$ and $P$ has only positive occurrences in $\sigma, R$ has only negative occurrences in $\sigma$.

By using $L$, we can restate this fact in the two fashions as follow:

$$
\vdash_{\mathscr{L}} \boldsymbol{\varphi} \supset \psi
$$

$\Leftrightarrow$

$$
\Psi_{3} \vdash_{L} \varphi^{1} \supset \psi^{2}
$$

$\stackrel{\leftrightarrow}{(6)}$

$$
\vdash_{\mathscr{L}} \boldsymbol{\varphi} \supset \sigma \text { and } \vdash_{\mathscr{L}} \sigma \supset \psi \quad \text { for some sentence } \sigma \in \Delta_{3} \text {, }
$$

where $\Delta_{3}$ is the least set satisfying

(a) $Q(x), \neg Q(x), E(x, y), \neg E(x, y), P(x), \neg R(x) \in \Delta_{3}$ for any free variables $x, y$,

(b) $\Delta_{3}$ is closed under $\wedge, \vee, \forall, \exists$,

or

$$
\vdash_{\mathscr{L}} \boldsymbol{\varphi} \supset \psi
$$

$\stackrel{\leftrightarrow}{(7)}$

$$
\Psi_{4} \vdash_{L} \phi^{1} \supset \psi^{2}
$$

$\stackrel{\leftrightarrow}{8}$

$\vdash_{\mathscr{P}} \boldsymbol{\varphi} \supset \sigma$ and $\vdash_{\mathscr{S}} \sigma \supset \psi$ for some sentence $\sigma \in \Delta_{4}$, where $\Delta_{4}$ is the least set satisfying 
(a) $Q\left(x_{1}\right), \neg Q\left(x_{1}\right), E\left(x_{1}, x_{2}\right), \neg E\left(x_{1}, x_{2}\right), P\left(x_{1}\right), \neg R\left(x_{1}\right) \in \Delta_{4}$ for any $x_{1}, x_{2}, \in X$,

(b) $\Delta_{4}$ is closed under $\wedge, V$,

(C) $\Delta_{4}$ is closed under $\forall$,

(d) $\Delta_{4}$ is closed under $\exists$.

\section{(3) Relativization theorem}

If $\boldsymbol{\varphi}$ is a formula in $\mathscr{L}$, by $\boldsymbol{\rho}^{U}, \boldsymbol{\phi}^{1, U}$ we shall denote the relativizations of $\boldsymbol{\varphi}$ and $\phi^{1}$ by $U$.

Let $\boldsymbol{\varphi}, \psi$ be two sentences in $\mathscr{L}$. Then

$$
\Leftrightarrow \quad \begin{aligned}
& (\exists u) U(u) \vdash_{\mathscr{L}(U)} \Phi^{U} \supset \psi \\
& \vdash_{\mathscr{L}} \varphi \supset \sigma \text { and } \vdash_{\mathscr{S}} \sigma \supset \psi \quad \text { for some existential sentence } \sigma .
\end{aligned}
$$

By using $L$, we can restate this fact in the following:

$$
(\exists u) U(u) \underset{\mathscr{L}_{(U)}}{\boldsymbol{q}^{U}} \supset \psi
$$

$\Leftrightarrow$

$$
\Psi_{5} \vdash_{L} \varphi^{1, U} \supset \Psi^{2}
$$

$\stackrel{\leftrightarrow}{(110)}$

$\vdash_{\mathscr{L}} \boldsymbol{\varphi} \supset \sigma$ and $\vdash_{\mathscr{L}} \sigma \supset \psi$ for some sentence $\sigma \in \Delta_{5}$, where $\Delta_{5}$ is the least set satisfying

(a) $P(\vec{x}), \rightarrow P(\vec{x}) \in \Delta_{5}$ for $P \in P C$, any $\vec{x}$,

(b) $\Delta_{5}$ is closed under $\wedge, \vee, \exists$.

(4) Los-Tarski's theorem on extension (cf. Los [19], Tarski [34])

Let $\boldsymbol{\phi}, \psi$ be sentences in $\mathscr{L}$. Then

Every extension of a model of $\varphi$ is a model of $\psi$

$\Leftrightarrow$

$\vdash_{\mathscr{L}} \boldsymbol{\rho} \supset \boldsymbol{\sigma}$ and $\vdash_{\mathscr{L}} \sigma \supset \psi$ for some existential sentence $\sigma$.

By using $L$,

Every extension of a model of $\boldsymbol{\phi}$ is a model of $\psi$

$\stackrel{\leftrightarrow}{11}$

$$
\Psi_{5} \vdash_{L} \varphi^{1, U} \supset \psi^{2}
$$

$\stackrel{\leftrightarrow}{(12)}$

$\vdash_{\mathscr{L}} \boldsymbol{\rho} \supset \boldsymbol{\sigma}$ and $\vdash_{\mathscr{S}} \sigma \supset \Psi$ for some sentence $\sigma \in \Delta_{5}$

or

Every extension of a model of $\boldsymbol{\rho}$ is a model of $\psi$ 


$$
\begin{aligned}
& \stackrel{\leftrightarrow}{(13} \quad \Psi_{6} \vdash_{L} \phi^{1} \supset \psi^{2} \\
& \stackrel{\leftrightarrow}{I^{2}} \quad \boldsymbol{F} \supset \sigma \text { and } \vdash_{\mathscr{L}} \sigma \supset \psi \text { for some sentence } \sigma \in \Delta_{6},
\end{aligned}
$$

where $\Delta_{6}$ is the least set satisfying

(a) $P(\vec{x}), \neg P(\vec{x}) \in \Delta_{6}$ for $P \in P C, \vec{x} \in X^{n}, n<\omega$,

(b) $\Delta_{6}$ is closed under $\wedge, \vee$,

(C) $\Delta_{6}$ is closed under $\exists$.

(5) Feferman-Kreisel's theorem on end-extension (cf. Feferman-Kreisel [8])

Let $\varphi, \psi$ be sentences in $\mathscr{L}$.

Then

Every end-extension of a model of $\phi$ is a model of $\psi$

$\Leftrightarrow$

$$
\vdash_{\mathscr{L}} \boldsymbol{\varphi} \supset \sigma \text { and } \vdash_{\mathscr{L}} \sigma \supset \psi \quad \text { for some } \cdots \text { sentence } \sigma .
$$

By using $L$,

Every end-extension of a model of $\varphi$ is a model of $\psi$

$\stackrel{\leftrightarrow}{(15)}$

$$
\Psi_{7} \vdash_{L} \varphi^{1, U} \supset \psi^{2}
$$

$\stackrel{\leftrightarrow}{(18)}$

$\vdash_{\mathscr{S}} \boldsymbol{\varphi} \supset \sigma$ and $\vdash_{\mathscr{S}} \sigma \supset \psi$ for some sentence $\sigma \in \Delta_{7}$, where $\Delta_{7}$ is the least set satisfying

(a) $P(\vec{x}), \rightarrow P(\vec{x}) \in \Delta_{7}$ for $P \in P C, \vec{x}$,

(b) $\Delta_{7}$ is closed under $\wedge, \vee, \exists$,

(C) If $\theta(x) \in \Delta_{7}$, then $(\forall v)(v<y \cdot \supset \theta(v)) \in \Delta_{7}$

or

Every end-extension of a model of $\boldsymbol{p}$ is a model of $\psi$

$\stackrel{\leftrightarrow}{(17)}$

$$
\Psi_{8} \vdash_{L} \phi^{1} \supset \psi^{2}
$$

$\overleftrightarrow{\leftrightarrow}$

$\vdash_{\mathscr{S}} \rho \supset \sigma$ and $\vdash_{\mathscr{L}} \sigma \supset \psi$ for some sentence $\sigma \in \Delta_{8}$, where $\Delta_{8}$ is the least set satisfying 
(a) $P(\vec{x}), \neg P(\vec{x}) \in \Delta_{\mathrm{g}}$ for $P \in P C, \vec{x} \in X^{n}, n<\omega$,

(b) $\Delta_{8}$ is closed under $\Lambda, \vee$,

(c) $\Delta_{8}$ is closed under $\exists$,

(d) If $\theta(x) \in \Delta_{8}$, then $(\forall v)\left(v<x_{1} \cdot \supset \theta(v)\right) \in \Delta_{8}$ for any $x_{1} \in X$.

(6) Lyndon's theorem on homomorphism (cf. Lyndon [18])

Let $\boldsymbol{\phi}, \psi$ be sentences in $\mathscr{L}$. Then

Every homomorphic image of a model of $\phi$ is a model of $\psi$ $\Leftrightarrow$

$\vdash_{\mathscr{L}} \boldsymbol{\phi} \supset \boldsymbol{\sigma}$ and $\vdash_{\mathscr{L}} \sigma \supset \psi$ for some positive sentence $\sigma$.

By using $L$,

Every homomorphic image of a model of $\boldsymbol{\rho}$ is a model of $\psi$

$\stackrel{\leftrightarrow}{(19)}$

$$
\Psi_{9} \vdash \varphi^{1} \supset \psi^{2}
$$

$\stackrel{\leftrightarrow}{(20)}$

$\vdash_{\mathscr{L}} \boldsymbol{\varphi} \supset \sigma$ and $\vdash_{\mathscr{L}} \sigma \supset \psi$ for some sentence $\sigma \in \Delta_{9}$, where $\Delta_{9}$ is the least satisfying

(a) $P(\vec{x}) \in \Delta_{9}$ for $P \in P C, \vec{x} \in X^{n}, n<\omega$,

(b) $\Delta_{\theta}$ is closed under $\wedge, \vee$,

(C) $\Delta_{\theta}$ is closed under $\forall$,

(C) $\Delta_{9}$ is closed under $\exists$.

(7) Keisler's theorem on retract (cf. Keisler [13])

Let $\boldsymbol{\varphi}, \psi$ be sentences in $\mathscr{L}$. Then

Every retract of a model of $\boldsymbol{\rho}$ is a model of $\psi$ $\Leftrightarrow$

$$
\vdash_{\mathscr{L}} \varphi \supset \sigma \text { and } \vdash_{\mathscr{L}} \sigma \supset \psi \text { for some.... sentence } \sigma, \quad \text { (cf, [13]). }
$$

By using $L$,

Every retract of a model of $\varphi$ is a model of $\psi$

$\stackrel{\Leftrightarrow}{(21)}$

$$
\Psi_{10} \vdash_{L} \phi^{1} \supset \psi^{2}
$$

$\stackrel{\Leftrightarrow}{(22)}$

$\vdash_{\mathscr{L}} \boldsymbol{\varphi} \supset \sigma$ and $\vdash_{\mathscr{L}} \sigma \supset \psi$ for some sentence $\sigma \in \Delta_{10}$, where $\Delta_{10}$ is the least set satisfying 
(a) $P(\vec{x}) \in \Delta_{10}$ for $P \in P C, \vec{x} \in X^{n}, n<\omega$,

(a) $P(\vec{y}), \rightarrow P(\vec{y}) \in \Delta_{10}$ for $P \in P C, \vec{y} \in Y^{n}, n<\omega$,

(b) $E(x, y) \in \Delta_{10}$ for $x \in X, y \in Y$,

(C) $\Delta_{10}$ is closed under $\wedge, \vee$,

(d) If $\theta(x) \in \Delta_{10}$ and $x \in X$, then $(\forall v) \theta(v) \in \Delta_{10}$,

(d)' If $\theta(y) \in \Delta_{10}$ and $y \in Y$, then $(\forall v) \theta(v) \in \Delta_{10}$,

(e) If $\theta(x) \in \Delta_{10}$ and $x \in X$, then $(\exists v) \theta(v) \in \Delta_{10}$.

All the equivalences (1), (3), (5), (7), (9), (11), (13), (15), (17), (19), (21) can be easily proved by completeness theorem of $L$ and some of them are to be proved directly. So, the essential difficulty in proving above theorems lies in the proofs of the equivalences (2), (4), (6), (8), (19), (12), (14), (16), (18), (20), (22).

It is here that "normal derivation theorem" in [27] can be used effectively. The remarkable resemblance between (2), $\cdots$, (2) and the features to be observed in all the constructions of $\Delta_{1}, \cdots, \Delta_{10}$ from $\Psi_{1}, \cdots, \Psi_{10}$ are the main impulses which gave rise to this research.

Now we formulate "interpolation problem and characterization problem" in these special logics $\mathscr{L}$ and $L$ (precise definitions for wider logics will be given in the section 1 in Chapter II, III and V).

Let $\Psi$ be a set of sentences in $L$.

Then

the invarient set $\operatorname{In}(\Psi)$ of $\Psi$ is the set of formulas $\theta$ in $\mathscr{L}$ such that $\Psi \vdash_{L} \theta^{1} \supset \theta^{2}$,

the $U$-invarient set $\operatorname{In}_{U}(\Psi)$ of $\Psi$ is the set of formulas $\theta(\vec{x})$ in $\mathscr{L}$ such that $\Psi \vdash_{L}(\forall \vec{u})\left(U(\vec{u})_{\wedge} \theta^{1, U}(\vec{u}) . \supset \theta^{2}(\vec{u})\right)$,

where $\vec{x}$ is a repetition-free enumeration of free variables in $\theta$, the $I$ invarient set $I n_{I}(\Psi)$ of $\Psi$ is the set of formulas $\theta(\vec{x}, \vec{y})$ in $\mathscr{L}$ such that $\Psi \vdash_{L}\left(\forall \vec{u}_{1}\right)\left(\forall \vec{v}_{1}\right)\left(\forall \vec{u}_{2}\right)\left(\forall \vec{v}_{2}\right)\left(I_{0}\left(\vec{u}_{1}, \vec{v}_{1}\right)_{\wedge} I_{1}\left(\vec{u}_{2}, \vec{v}_{2}\right)_{\wedge} \theta^{1}\left(\vec{u}_{1}, \vec{u}_{2}\right) . \supset \theta^{2}\left(\vec{v}_{1}, \vec{v}_{2}\right)\right)$,

where $\vec{x}$ is a repetition-free enumeration of free variables of $\theta$ in $X$ and $\vec{y}$ is a repetition-free enumeration of free variables of $\theta$ in $Y$.

A subset $\Delta$ of $I n(\Psi)\left(I n_{U}(\Psi), I n_{I}(\Psi)\right)$ is said to be a characterization (U-characterization, I-characterization) set of $\Psi$ if for any $\theta \in \operatorname{In}(\Psi)\left(\operatorname{In}_{U}(\Psi), \operatorname{In}_{I}(\Psi)\right)$, there is a formula $\theta^{\prime} \in \Delta$ such that $\vdash_{\mathscr{L}} \theta \equiv \theta^{\prime}$ and every free variable in $\theta^{\prime}$ occurs in $\theta$.

$\Psi$ is interpolatable if for any $\boldsymbol{\phi}, \psi$ in $\mathscr{L}$,

$\Psi \vdash_{L} \boldsymbol{\phi}^{1} \supset \psi^{2}$ implies $\vdash_{\mathscr{L}} \boldsymbol{\varphi} \supset \sigma$ and $\vdash_{\mathscr{L}} \sigma \supset \psi$ for some $\sigma \in \operatorname{In}(\Psi)$ such that every free variable in $\sigma$ occurs both in $\varphi$ and in $\psi$. 
$\Psi$ is $U$-interpolatable if for any finite sequence $\vec{x}$ of distinct free variables and any $\varphi(\vec{x}), \varphi(\vec{x})$ in $\mathscr{L}$,

$$
\begin{aligned}
& \Psi \vdash_{L}(\forall \vec{u})\left(U(\vec{u})_{\wedge} \phi^{1, U}(\vec{u}) . \supset \psi^{2}(\vec{u})\right) \text { implies } \vdash_{\mathscr{L}} \boldsymbol{\varphi} \supset \sigma \text { and } \vdash_{\mathscr{L}} \sigma \supset \psi \text { for some } \\
& \sigma \in \operatorname{In}_{U}(\Psi) \text { such that every free variable in } \sigma \text { occurs in } \vec{x} \text {. }
\end{aligned}
$$

$\Psi$ is $I$-interpolatable if for any finite sequence $\vec{x}$ of distinct free variables in $X$, any finite sequence $\vec{y}$ of distinct free variables in $Y$ and any $\varphi(\vec{x}, \vec{y}), \psi(\vec{x}, \vec{y})$ in $\mathscr{L}$,

$$
\Psi \vdash_{L}\left(\forall \vec{u}_{1}\right)\left(\forall \vec{v}_{1}\right)\left(\forall \vec{u}_{2}\right)\left(\forall \vec{v}_{2}\right)\left(I_{0}\left(\vec{u}_{1}, \vec{v}_{1}\right)_{\wedge} I_{1}\left(\vec{u}_{2}, \vec{v}_{2}\right)_{\wedge} \phi^{1}\left(\vec{u}_{1}, \vec{u}_{2}\right) . \supset \psi^{2}\left(\vec{v}_{1}, \vec{v}_{2}\right)\right)
$$

implies $\vdash_{\mathscr{L}} \boldsymbol{} \supset \sigma$ and $\vdash_{\mathscr{L}} \sigma \supset \psi$ for some $\sigma \in \operatorname{In}(\psi)$ such that every free variable in $\sigma$ occurs in $\vec{x}$ or $\vec{y}$.

Notice that in the definitions of Interpolatability or I-interpolatability, the latter conditions imply the former one, but in the definition of U-interpolatability, this does not hold true for the reason that "relativization" does not preserve "provability".

Then

\section{Interpolation (U-interpolation, I-interpolation) problem}

"What kind of $\Psi$ is interpolatable ( $U$-interpolatable, $I$-interpolatable)?"

\section{Characterization (U-characterization, I-characterization) problem}

"Find a syntactically simple characterization ( $U$-characterization, $I$-characterization) set of $\Psi^{\prime \prime}$.

In this paper, we shall take up these problems in more general situations including many predicate symbols $\left\{I_{i}\right\}_{i<\omega}$ and more general logics such as second order logic, infinitary logic, many valued logic....etc. We want to let $\mathscr{L}$ take as much logic as possible. So, instead of making the explicit definition of $\mathscr{L}$, we shall make explicit the requirements on $\mathscr{L}$ which are to include the requirements on object logics in [27].

Now we shall summarize the method to be used in the partial solutions of above problems.

First of all, let us take a glance at some properties of $\operatorname{In}(\Psi), \operatorname{In}_{U}(\Psi), \operatorname{In}_{I}(\Psi)$. Then we can easily see that

$\operatorname{In}(\psi)$ is closed under $\wedge, \vee, \forall, \exists$ (see (b) in $\Delta_{1}, \Delta_{3}$ ),

$\operatorname{In}_{U}(\Psi)$ is closed under $\wedge, \vee, \exists$ (see (b) in $\Delta_{5}, \Delta_{7}$ ),

$\operatorname{In}(\Psi)$ is closed under $\wedge, \vee \quad$ (see (b) in $\Delta_{2}, \Delta_{4}, \Delta_{6}, \Delta_{8}, \Delta_{9}$, (C) in $\Delta_{10}$ ).

These facts mean that

in the case of "Interpolation problem and characterization problem" we can not hope to controle logical connectives and quantifiers in $\operatorname{In}(\Psi)$ from 
the outside,

in the case of " $U$-interpolation problem and $U$-characterization problem" we can only hope to controle $\forall$ in $\operatorname{In}_{U}(\Psi)$ from the outside, in the case of " $I$-interpolation problem and $I$-characterization problem" we can controle $\forall, \exists$ in $\operatorname{In}_{I}(\Psi)$ from the outside.

Next, we shall look more closely into the constructions $\Delta_{1}, \cdots, \Delta_{10}$ from $\Psi_{1}$, $\cdots, \Psi_{10}$.

Then we can easily see what kinds of effects each type of sentences in $\Psi$ has on $\operatorname{In}(\Psi), \operatorname{In}_{U}(\Psi), \operatorname{In}_{I}(\Psi)$.

For example,

$$
\begin{gathered}
(\forall \vec{u})\left(\theta^{1}(\vec{u}) \supset \theta^{2}(\vec{u})\right) \in \Psi \text { makes } \begin{array}{c}
\theta(\vec{x}) \in \operatorname{In}(\Psi), \\
\text { cf. (a) in } \Delta_{1}, \Delta_{3},
\end{array} \\
(\forall \vec{u})\left(U(\vec{u})_{\wedge} \theta^{1, U}(\vec{u}) . \supset \theta^{2}(\vec{u})\right) \in \Psi \text { makes } \theta(\vec{x}) \in \operatorname{In} n_{U}(\Psi), \\
\text { cf. (a) in } \Delta_{5}, \Delta_{7}, \\
\left(\forall \vec{u}_{1}\right)\left(\forall \vec{v}_{1}\right)\left(\forall \vec{u}_{2}\right)\left(\forall \vec{v}_{2}\right)\left(I_{0}\left(\vec{u}_{1}, \vec{v}_{1}\right)_{\wedge} I_{1}\left(\vec{u}_{2}, \vec{v}_{2}\right)_{\wedge} \theta^{1}\left(\vec{u}_{1}, \vec{u}_{2}\right) . \supset \theta^{2}\left(\vec{v}_{1}, \vec{v}_{2}\right)\right) \\
\text { makes } \theta(\vec{x}, \vec{y}) \in \operatorname{In}_{I}(\Psi) \text { for any } \vec{x} \in X^{n}, \vec{y} \in Y^{m}, \\
\text { cf. (a) in } \Delta_{2}, \Delta_{4}, \Delta_{6}, \Delta_{8}, \Delta_{9}, \Delta_{10}, \text { (a)', (b) in } \Delta_{10},
\end{gathered}
$$

$(\forall u)(\exists v) I_{0}(u, v) \in \Psi$ makes $(\exists u) \theta(u) \in \operatorname{In}_{I}(\Psi)$ for any $\theta(x) \in \operatorname{In}_{I}(\Psi), x \in X$,

cf. (d) in $\Delta_{2}, \Delta_{4}, \Delta_{9}$, (C) in $\Delta_{10}$,

$(\forall v)(\exists u) I_{0}(u, v) \in \Psi$ makes $(\forall u) \theta(u) \in I n_{I}(\Psi)$ for any $\theta(x) \in \operatorname{In}_{I}(\Psi), x \in X$,

cf. (C) in $\Delta_{2}, \Delta_{4}, \Delta_{6}, \Delta_{8}, \Delta_{9}$, (d) in $\Delta_{10}$,

$(\forall v)(\exists u) I_{1}(u, v) \in \Psi$ makes $(\forall u) \theta(u) \in \operatorname{In}_{I}(\Psi)$ for any $\theta(y) \in \operatorname{In}_{I}(\Psi), y \in Y$,

$$
\text { cf. (d) in } \Delta_{10} \text {, }
$$

$$
\begin{gathered}
\left(\forall u_{1}\right)\left(\forall v_{1}\right)\left(\exists u_{2}\right)\left(U\left(v_{1}\right)_{\wedge} u_{1}<^{2} v_{1} \cdot \supset . U\left(u_{2}\right)_{\wedge} E^{2}\left(u_{1}, u_{2}\right)\right) \in \Psi \\
\text { makes }(\forall v)(v<y \supset \theta(v)) \in I n_{U}(\Psi) \text { for } \theta(x) \in I n_{U}(\Psi), \\
\text { cf. (C) in } \Delta_{7}, \\
\left(\forall u_{1}\right)\left(\forall v_{1}\right)\left(\forall v_{2}\right)\left(\exists u_{2}\right)\left(v_{2}<^{2} v_{1 \wedge} I_{0}\left(u_{1}, v_{1}\right) . \supset I_{0}\left(u_{2}, v_{2}\right)\right) \in \Psi \\
\text { makes }(\forall v)\left(v<x_{1} \supset \theta(v)\right) \in I n_{I}(\Psi) \text { for } \theta(x) \in I n_{I} \Psi, x, x_{1} \in X, \\
\text { cf. (d) in } \Delta_{8} .
\end{gathered}
$$

Then we classify sentences into some types and define primitive ( $U$-primitive, $I$-primitive) sentences and primitive ( $U$-primitive, $I$-primitive) sets.

According to the above correspondences between sentences in $\Psi$ and their effects on $\operatorname{In}(\Psi), \operatorname{In}_{U}(\Psi), I n_{I}(\Psi)$, we can associate in a uniform method a set $\Delta(\Psi)$ $\left(\Delta_{U}(\Psi), \Delta_{I}(\Psi)\right)$ for each primitive (U-primitive, $I$-primitive) set $\Psi$. 
Then our main theorems say that

Every primitive set is interpolatable,

Every $U$-primitive set is $U$-interpolatable,

Every $I$-primitive set is $I$-interpolatable,

$\Delta(\Psi)$ is a characterization set of $\Psi$ for each primitive set $\Psi, \Delta_{U}(\Psi)$ is an

$U$-characterization set of $\Psi$ for each $U$-primitive set $\Psi$,

$\Delta_{I}(\Psi)$ is an $I$-characterization set of $\Psi$ for each $I$-primitive set $\Psi$.

These theorems, of course, imply our examples as is made clear in the following way:

$\Psi_{1}, \Psi_{3}$ are primitive sets and $\Delta\left(\Psi_{1}\right)=\Delta_{1}, \Delta\left(\Psi_{3}\right)=\Delta_{3}$,

$\Psi_{5}, \Psi_{7}$ are $U$-primitive sets and $\Delta_{U}\left(\Psi_{5}\right)=\Delta_{5}, \Delta_{U}\left(\Psi_{7}\right)=\Delta_{7}$.

$\Psi_{2}, \Psi_{4}, \Psi_{6}, \Psi_{8}, \Psi_{4}, \Psi_{10}$ are $I$-primitive sets and $\Delta_{I}\left(\Psi_{2}\right)=\Delta_{2}, \Delta_{I}\left(\Psi_{4}\right)=\Delta_{4}$,

$$
\Delta_{I}\left(\Psi_{6}\right)=\Delta_{6}, \Delta_{I}\left(\Psi_{8}\right)=\Delta_{8}, \Delta_{I}\left(\Psi_{9}\right)=\Delta_{9}, \Delta_{I}\left(\Psi_{10}\right)=\Delta_{10} .
$$

Although our theorems are far from complete solutions of the above problems, they include almost all the results in these fields that have been obtained before in both model theory and proof theory.

In the "interpolation problem" or in the "U-interpolation problem", the morphism between the universe corresponding to formulas of the form $\theta^{1}$ or $\theta^{1, U}$ and the universe corresponding to formulas of the form $\theta^{2}$ is given by the metamathematical identity relation i.e. $x \rightarrow x$.

On the other hand, in the " $I$-interpolation problem" morphisms are given by predicate constants $I_{0}, I_{1}$.

Hence " $I$-interpolation theorem and $I$-characterization theorem" can be considered as a syntactical counterpart of "preservation theorem" in model theory.

A set $\Psi$ of sentences in $L$ can be thought of as a set of axioms for a specific morphism in the morphism logic $L$, e.g. $\Psi_{6}$ is the set of axioms for embedding, $\Psi_{9}$ the set of axioms for homomorphism and $\Psi_{10}$ the set of axioms for retract.

Then from our main theorems is it inferred that we can know the preservation theorem of an algebraic relation (i.e. morphism) by looking at the set of axioms $\Psi$ of this morphism only and that the characterization set $\Delta_{I}(\Psi)$ can be constructed only from the syntactical structures of $\Psi$ itself.

As far as the author knows, the methods of proofs of preservation theorems can be classified into the following six groups:

Group 1 Diagram method,

Group 2 Interpolation theorems for one sorted logic,

Group 3 Special models and saturated models,

Group 4 Infinitely long formulas and saturated models,

Group 5 Smullyan-Makkai's method,

Group 6 Interpolation theorems for many sorted logic. 
Diagram method was used in the beginning of model theory by Chang [4], Keisler [9], Los [19], and Tarski [34] in the first order logic. The methods in group 2 were developed by R. Lyndon [17], [18] and applied by Lopez-Escobar [20], Malitz [23] to model theory on $L_{\omega_{1} \omega}$.

The methods in group 3 are the most ordinal ones in model theory on the first order logic or continuous logic and used in Chang-Keisler [5], Keisler[10], [11], Weglorz [35], Motohashi [25] (cf. [29]).

The methods in group 4 were developed by Keisler [12], [13].

The methods of the four groups above were successfully used in model theory on the finitary first order logic or continuous logic, but not in model theories on other logics.

The Smyllyan-Makkai's method was developed by Makkai [22] and used to obtain a great many results in model theory on $L_{\omega_{1} \omega}$ by himself and Nebres [30], (cf. Keisler [14]).

The methods in group 6 were developed by Feferman [6], [7].

To summerize, ours can be considered as an essentially new method and has the following advantages;

(1) It can be expressed in a completely syntactical form so that we can get uniform ways of treatment of interpolation theorem, relativization theorem and preservation theorems in many logics such as non finitary logic, non first order logic, non classical logic, etc.

(2) Up to a point we can know whether $\Psi$ is interpolatable or not by syntactical structures of sentences in $\Psi$.

(3) We can see the syntactical structure of interpolating formulas (preservation formulas) by looking at the axioms themselves, more precisely the syntactical structures of the axioms themselves.

(4) We need not make efforts any more to eliminate "cut rules" in many systems to prove interpolation theorems, e.g. a complicated treatment of equality axioms in [7] will not be necessary any more.

(5) Function symbols need not be eliminated in the object logics in proving interpolation theorem because in the morphism logic no function symbols appear outside of formulas in object logics (cf. [36]).

As applications of our theorems, we have

(6) New proofs of many known preservation theorems in some logics and their extensions to other logics such as positive second order logic and non continuous logic.

(7) A new proof of Svenonius' definability theorem and its extension to $L_{\omega_{1} \omega}$. (cf. [26]).

(8) A reduction of Feferman's interpolation theorem in many sorted logic to that of single sorted logic, (cf. [6] ). 
The details of these applications except (8) will be mentioned in sequels of this paper.

This paper consists of five Chapters.

Chapter I deals with object logics and morphism logics used in the following Chapters.

Chapter II deals with "interpolation problem and characterization problem". Chapter III deal with " $U$-interpolation problem and $U$-characterization problem". Chapter IV deal with "Obstruction problem of $\Psi$-extension",

which treats in a generalized way the following type problems: "For any sentences $\boldsymbol{\phi}, \psi$ in $\mathscr{L}$, find a syntactical necessary and sufficient condition that there be no models $\mathscr{A}, \mathscr{B}$ such that $\mathscr{A}$ is a model of $\boldsymbol{\varphi}, \mathscr{B}$ is a model of $\psi$ and $\mathscr{B}$ is a proper end-extension of $\mathscr{A}$.

Chapter V deals with " $I$-interpolation problem and $I$-characterization problem".

Finally we show an application of our theorems to the theory of definition (8) in the applications of our theorems).

It is well-known that Beth's definability theorem (cf. Beth [1]) can be directly proved from Craig's interpolation theorem (cf. Maehara [24]). But, up to date, there has been no similar method in which we can prove Svenonius' definability theorem (cf. Motohashi [26]).

This is the main reason why we could not extend Svenonius' theorem to $L_{\omega_{1} \omega}$, although Beth's theorem was already extended to $L_{\omega, \omega}$ by Lopez-Escobar.

But, by using our theorems, we can easily prove Svenonius' theorem just as we can prove Beth's theorem by using Craig's theorem.

Suppose $P$ is an unary predicate constant not in $\mathscr{L}$ and $\mathscr{L}(P)$ is the first order logic obtained from $\mathscr{L}$ by adding $P$.

For any $\mathscr{L}(P)$-structure $\mathscr{A}$, by $\mathscr{A} \uparrow \mathscr{L}$ we shall denote the reduct of $\mathscr{A}$ to $\mathscr{L}$.

Let $\Psi$ be the set of following sentences;

$$
\begin{aligned}
& (\forall u)(\exists v) I_{0}(u, v),(\forall v)(\exists u) I_{0}(u, v), \\
& (\forall u)(\exists v) I_{1}(u, v),(\forall v)(\exists u) I_{1}(u, v), \\
& (\forall \vec{u})(\forall \vec{v})\left(I_{0}(\vec{u}, \vec{v})_{\wedge} \phi^{1}(\vec{u}) . \supset \phi^{2}(\vec{v})\right), \\
& (\forall \vec{u})(\forall \vec{v})\left(I_{1}(\vec{u}, \vec{v})_{\wedge} \psi^{1}(\vec{u}) . \supset \psi^{2}(\vec{v}),\right.
\end{aligned}
$$

where $\varphi(\vec{x})$ is an atomic formula or its negation in $\mathscr{L}$ and $\vec{x} \in X^{n}, n<\omega$, $\psi(\vec{y})$ is an atomic formula or its negaton in $\mathscr{L}(P)$ and $y \in Y^{m}, m<\omega$.

And $\Delta$ be the least set satisfying,

(a) $\boldsymbol{\phi}(\vec{x}) \in \Delta$ if $\boldsymbol{\varphi}(\vec{x})$ is an atomic formula or its negation in $\mathscr{L}$ and $\vec{x} \in X^{n}$, $n<\omega$,

(b) $\psi(\vec{y}) \in \Delta$ if $\psi(\vec{y})$ is an atomic formula or its negation in $\mathscr{L}(P)$ and $\vec{y} \in Y^{m}$, $m<\omega$, 
(C) $\Delta$ is closed under $\Lambda, \vee, \forall, \exists$.

Then $\Psi$ is $I$-primitive and $\Delta_{I}(\Psi)=\Delta$.

Hence $\Psi$ is $I$-interpolatable and $\Delta$ is an $I$-characterization set of $\Psi$.

Also, we can easily prove that every formula $\theta$ in $\Delta$ is equivalent to a formula of the form

$$
\left(\theta_{11}{ }^{\vee} \theta_{21}\right)_{\wedge} \cdots{ }_{\wedge}\left(\theta_{1 k}{ }^{\vee} \theta_{2 k}\right)
$$

where all the free variables in $\theta_{1 i}$ belong to $X$, all the free variables in $\theta_{2 i}$ belong to $Y$ and every $\theta_{1 i}$ does not contain $P, i=1,2, \cdots, k$, (Notice that $P(y) \in \Delta$ for any $y \in Y$. But $P(x) \notin \Delta$ for any $x \in \dot{X})$.

Let $T$ be a sentence in $\mathscr{L}(P)$ and $x$ is a free variable in $X$. Then

$$
\begin{aligned}
& \mathscr{A}\lceil\mathscr{L}=\mathscr{B}\lceil\mathscr{L} \text { and } \mathscr{A} \cong \mathscr{B} \text { imply } \mathscr{A}=\mathscr{B} \text { for any models } \mathscr{A}, \mathscr{B} \text { of } T \\
& \text { 다 } \\
& \Psi, T^{1}, T^{2} \vdash_{L}(\forall u)(\forall v)\left(I_{0}(u, v)_{\wedge} P^{1}(u) . \supset P^{2}(v)\right) \\
& \text { c) } \\
& \Psi \vdash_{L} I_{0}(x, x), T^{1}, P^{1}(x) \rightarrow\left(T^{2} \supset P^{2}(x)\right) \\
& \text { 다 } \\
& T \underset{\mathscr{S}^{(P)}}{\vdash} P(x) \equiv \theta(x), \text { for some } \theta(x) \in \Delta, \\
& \text { where } \theta(x)=\left(\theta_{11}(x)^{\vee}\left(\theta_{21}\right)\right)_{\wedge} \cdots{ }_{\wedge}\left(\theta_{1 k}(x)^{\vee}\left(\theta_{2 k}\right)\right) \\
& \text { 도 } \\
& T \underset{\mathscr{L}_{(P)}}{\vdash} \rightarrow \theta_{2 i} \supset\left(P(x) \supset \theta_{1 i}(x)\right), \quad i=1, \cdots, k \\
& T \underset{\mathscr{L}(P)}{\vdash} \bigwedge_{i=1}^{k}\left(\theta_{1 i}(x)^{\vee} \theta_{2 i}\right) . \supset P(x) \\
& \text { 드 } \\
& \underset{\mathscr{S}_{(P)}}{\vdash} \theta_{2 J_{\wedge}} P(x) . \supset \theta_{1 J}(x) \\
& T \underset{\mathscr{P}^{(}(P)}{\vdash} \theta_{2 J_{\Lambda}} \theta_{1 J}(x) . \supset P(x) \\
& \text { for }\left\{\begin{array}{l}
J \subseteq\{1,2, \cdots, k\} \\
\theta_{1 J}(x)=\bigwedge_{i \in J}^{\wedge} \theta_{1 i}(x) \\
\theta_{2 J}=\bigwedge_{i \in J} \rightarrow \theta_{2 i \wedge} \bigwedge_{i \notin J}^{\wedge} \theta_{2 i}
\end{array}\right. \\
& \text { 다 } \\
& T \underset{\mathscr{S}_{(P)}}{\vdash} \theta_{2 J} \supset(\forall u)\left(P(u) \equiv \theta_{1 J}(u)\right), \quad J \subseteq\{1,2, \cdots, k\} \\
& \text { 다 } \\
& T \underset{\mathscr{L}_{(P)}}{\vdash} \vee \theta_{2 J} \supset \underset{J}{\vee}(\forall u)\left(P(u) \equiv \theta_{1 J}(u)\right)
\end{aligned}
$$

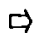

$$
\begin{aligned}
& \left.T \underset{\mathscr{S}_{(P)}}{\vdash} \underset{J}{\vee}(\forall u)\left(P(u) \equiv \theta_{1 J}(u)\right), \quad \text { (because } \underset{\mathscr{S}_{(P)}}{\leftarrow} \quad \underset{J}{ } \quad \theta_{2 J}\right) \\
& \text { a }
\end{aligned}
$$

This proved Svenonius' theorem, i.e.

$$
\mathscr{A} \uparrow \mathscr{L}=\mathscr{B} \uparrow \mathscr{L} \text { and } \mathscr{A} \cong \mathscr{B} \text { imply } \mathscr{A}=\mathscr{B} \text { for any models } \mathscr{A}, \mathscr{B} \text { of } T
$$


$\Leftrightarrow$

$$
T \underset{\mathscr{L}_{(P)}}{\vdash} \bigvee_{j=1}^{m}(\forall u)\left(P(u) \equiv \theta_{j}(u)\right) \text { for some } \theta_{1}(x) \cdots \theta_{m}(x) \text { in } \mathscr{L} \text {. }
$$

\section{Ghapter I. Object logic and Morphism logic}

In this Chapter we shall introduce some specific object logics and morphism logics which will be used in the following Chapters.

\section{$\S 1$ Object logics $\mathscr{L}, \mathscr{L}^{1}, \mathscr{L}^{2}$.}

The logic $\mathscr{L}$, we shall consider in this paper, satisfies the following requirements (1)-23).

\subsection{Requirements on $\mathscr{L}$.}

(1) $\mathscr{L}$ has a countably infinite set $F V$ of free individual variables denoted by $x$, $y, w$ (with or without suffixes).

(2) $\mathscr{L}$ has an uncountably infinite set $B V$ of bound individual variables denoted by $u, v, \cdots$ (with or without suffixes).

(3) $\mathscr{L}$ has a non empty set $P C$ of predicate constants denoted by $P, Q, \cdots$ (with or without suffixes).

(4) As connectives and quantifiers, $\mathscr{L}$ has at least $\rightarrow$ (negation), $\wedge$ (countable conjunction), $\vee$ (countable disjunction), $\forall$ (universal quantification) and $\exists$ (existential quantification).

(5) $\mathscr{L}$ has two propositional constants $T$ (truth) and $\perp$ (false).

In the following of this paper, if we simply say "variables" then they always mean "individual variables".

Let $F M$ be the set of formulas in $\mathscr{L}$ and $P F M$ be the set of provable formulas in $\mathscr{L}$. By $\varphi, \psi, \theta \cdots$ (with or without suffixes) we shall denote formulas in $\mathscr{L}$.

(6) Every formula in $\mathscr{L}$ has only finitely many free individual variables and at least one symbol without (individual) variables or connectives or quantifiers.

(7) If $\theta(\vec{x}) \in F M(\theta(\vec{x}) \in P F M)$ and $\vec{x}, \vec{t}$ are two sequences of the same length such that $\vec{x}$ is a sequence of distinct free variables, $l$ is a sequence of individual terms, then $\theta(\vec{t}) \in F M(\theta(\vec{t}) \in P F M)$.

(8) If $\theta \in F M$ then $\rightarrow \theta \in F M$.

(9) If $\Phi$ is a non empty countable set of formulas in $\mathscr{L}$ such that only finitely many free variables occur in $\Phi$ then $\wedge \Phi, \vee \Phi \in F M$.

(11) If $\theta(\vec{x}) \in F M$ and $v$ does not occur in $\theta(x)$, then

$(\forall v) \theta(v),(\exists v) \theta(v) \in F M$.

(11)

$$
\top, \neg \perp \in P F M \text {. }
$$

(12)

$\vee\{\neg \theta, \theta\} \in P F M \quad$ for each $\theta \in F M$. 
No. 2

(13) If $\Phi_{0} \subseteq \Phi, \vee \Phi_{0} \in P F M, \vee \Phi \in F M$, then $\vee \Phi \in P F M$.

(14) If $\vee \Phi^{\cup}\{\theta\} \in P F^{\prime} M$ and $\vee \Phi^{\cup}\{\neg \theta\} \in P F M$ then $\vee \Phi \in P F M$.

(15) If $\vee \Phi^{\cup}\{\theta\} \in P F M$ then $\vee \Phi^{\cup}\{\neg \neg \theta\} \in P F M$.

(16) If $\vee \Phi^{\cup}\{\neg \theta\} \in P F M$ for some $\theta \in \Phi_{1}$ and $\wedge \Phi_{1} \in F M$ then

$$
\vee \Phi^{\cup}\left\{\neg \wedge \Phi_{1}\right\} \in P F M \text {. }
$$

(17D) If $\vee \Phi^{\cup}\{\theta\} \in P F M$ for all $\theta \in \Phi_{1}$ and $\wedge \Phi_{1} \in F M$ then $\vee \Phi^{\cup}\left\{\wedge \Phi_{1}\right\} \in P F M$.

(18) If $\vee \Phi \cup\{\neg \theta\} \in P F M$ for all $\theta \in \Phi_{1}$ and $\vee \Phi_{1} \in F M$ then $\Phi^{\cup}\left\{\neg \vee \Phi_{1}\right\} \in P F M$.

(19) If $\vee \Phi^{\cup}\{\theta\} \in P F M$ for some $\theta \in \Phi_{1}$ and $\vee \Phi_{1} \in F^{\prime} M$ then $\vee \Phi^{\cup}\left\{\vee \Phi_{1}\right\} \in P F M$.

(20) If $\vee \Phi^{\cup}\left\{\neg \theta\left(\begin{array}{l}x \\ y\end{array}\right)\right\} \in P F M$ then $\vee \Phi^{\cup}\{\neg(\forall v) \theta(v)\} \in P F M$.

(21) If $\vee^{\cup}\{\theta(x)\} \in P F M$ and $x$ does not appear in $\Phi$, then

$$
\vee \Phi^{\cup}\{(\forall v) \theta(v)\} \in P F M .
$$

$$
\vee \Phi^{\cup}\{\neg(\exists v) \theta(v)\} \in P F M .
$$

If $\Phi$ is a finite set $\left\{\varphi_{1}, \cdots \varphi_{n}\right\}$, we shall write $\wedge \Phi$ by $\boldsymbol{\varphi}_{\wedge} \cdots{ }_{\wedge} \boldsymbol{\varphi}_{n}$ and $\vee \Phi$ by $\boldsymbol{\rho}_{\mathbf{1}}{ }^{\vee} \ldots{ }^{\vee} \boldsymbol{\varphi}_{\boldsymbol{n}}$.

We shal use $\supset$ (implication), 三(equivalence) as abbreviations as usual. Of course " $\vdash_{\mathscr{S}} \boldsymbol{\phi}$ " means " $\phi \in P F M "$ ".

From $\mathscr{L}$, we get two object logics $\mathscr{L}^{1}, \mathscr{L}^{2}$ by attaching 1 or 2 to every symbol, except variables, connectives or quantifiers, in every formula in $\mathscr{L}$ respectively. By $\theta^{1}, \theta^{2}$ we shall denote the formulas obtained from $\theta$ by applying the operation stated above respectively.

For example $((\forall v) P(x, v))^{1}$ is $(\forall v) P^{1}(x, v),(\neg \top)^{1}$ is $\neg \top^{1}$ etc. Moreover if $\mathscr{L}$ has a second order variable $\alpha$, then

$$
((\forall \alpha)(\exists v) \alpha(v))^{1} \text { is }\left(\forall \alpha^{1}\right)(\exists v) \alpha^{1}(v) \text {, etc.... . }
$$

Let $F M^{1}$ be the set of formulas in $\mathscr{L}^{1}$ and $P F M^{1}$ be the set of provable formulas in $\mathscr{L}^{1}$. Similarly $F M^{2}, P F M^{2}$ can be defined.

Then obviously $\left\{\mathscr{L}^{1}, \mathscr{L}^{2}\right\}$ satisfies the requirements on object logics in Motohashi [27] by 1.1 (6)-(11).

Sometimes formulas in $F M^{1}$ will be called 1 -formulas and formulas in $F M^{2}, 2$ formulas

By an $\mathscr{L}$-sequent, we shall mean a configuration of the form $\Phi_{1} \rightarrow \Phi_{2}$ where $\Phi_{1}, \Phi_{2}$ are countable (possibly empty) sets of formulas in $\mathscr{L}$ such that only finitely many free variables occur in $\Phi_{1} \cup_{\Phi_{2}}$. Suppose $\Phi_{1 \rightarrow \Phi_{2}}$ is an $\mathscr{L}$-sequent. Then 


$$
\begin{aligned}
& \vdash_{\mathscr{L}} \Phi_{1} \rightarrow \Phi_{2} \text { means that } \vee\left\{\neg \theta ; \theta \in \Phi^{1}\right\} \cup \Phi_{2} \in P F M \text { if } \Phi_{1} \cup \Phi_{2} \neq \phi \\
& \text { and } \perp \in P F M \text { if } \Phi_{1} \cup \Phi_{2}=\phi,
\end{aligned}
$$

(where $\phi$ is the empty set).

\section{§ 2 Morphism logic $L=L\left(\mathscr{L}^{1}, \mathscr{L}^{2}\right)$.}

From object logics $\left\{\mathscr{L}^{1}, \mathscr{L}^{2}\right\}$ we define the morphism logic $L=L\left(\mathscr{L}^{1}, \mathscr{L}^{2}\right)$ by the method in [27].

Then the set of formulas in $L$ (denoted by $F M^{L}$ ) is given by the following rules:

(1) $\theta^{1}, \theta^{2}$ are formulas in $L$ for each $\theta \in F M$.

(2) If $F$ is a formula in $L$, then $\rightarrow F$ is a formula in $L$.

(3) If $K$ is a non empty countable set of formulas in $L$ such that only finitely many free variables occur in $K$, then $\wedge K, \vee K$ are formulas in $L$.

(4) If $F(x)$ is a formula in $L$ and $v$ does not occur in $F(x)$, then

$$
(\forall v) F(v),(\exists v) F(v) \text { are formulas in } L .
$$

(5) All the formulas in $L$ are obtained from (1)-(4).

Let $\Gamma \rightarrow \Theta$ be a sequent in $L$ i.e. $\Gamma$ and $\Theta$ are countable (possibly empty) sets of formulas in $L$ sach that only finitely many free variables occur in $\Gamma^{\cup} \Theta$.

Then by $\Gamma(1) \rightarrow \Theta(1)$ we shall mean the $\mathscr{L}$-sequent obtained from $\Gamma \rightarrow \Theta$ by deleting every non 1-formula and then replacing every $\theta^{1}$ by $\theta$. Similarly $\Gamma(2) \rightarrow \Theta(2)$ is defined. Then the conditions 1.1 (12)-(28) make us possible to prove the following proposition 2.1 by using normal derivation theorem in [27].

\subsection{Proposition.}

Suppose $\Gamma, \Theta$ consist of only 1-formulas or 2-formulas.

Then

$$
\vdash_{L} \Gamma \rightarrow \Theta \text { implies } \vdash_{\mathscr{L}} \Gamma(1) \rightarrow \Theta(1) \text { or } \vdash_{\mathscr{L}} \Gamma(2) \rightarrow \Theta(2)
$$

Proof By the induction on a normal derivation $\mathscr{D}$ of $\Gamma \rightarrow \Theta$. We only treat the case that the last rule of $\mathscr{D}$ is a cut rule.

Then $\mathscr{D}$ has the form

$$
\begin{array}{ccc}
\downarrow & \downarrow \mathscr{D}_{1} & \downarrow \mathscr{D}_{2} \\
\text { where } & \Gamma_{1} \rightarrow \Theta_{1} & \Gamma_{2} \rightarrow \Theta_{2} \\
(C, F) \frac{\Gamma_{1}, \Gamma_{2}-F \rightarrow \Theta_{1}-F, \Theta_{2}}{\Gamma_{1}, \Gamma_{2}-F=\Gamma_{1} \cup\left(\Gamma_{2}-\{F\}\right)=\Gamma} \\
\Theta_{1}-F, \Theta_{2}=\left(\Theta_{1}-\{F\}\right)^{\cup} \Theta_{2}=\Theta .
\end{array}
$$

By the hypotheses of induction, $\vdash_{\mathscr{L}} \Gamma_{1}(1) \rightarrow \Theta_{1}(1)$ or $\vdash_{\mathscr{S}} \Gamma_{1}(2) \rightarrow \Theta_{1}(2)$ and $\underset{\mathscr{S}}{\vdash} \Gamma_{2}(1)$ 
$\Theta_{2}(1)$ or $\underset{\mathscr{L}}{\vdash} \Gamma_{2}(2) \rightarrow \Theta_{2}(2)$.

Since $\mathscr{D}$ is normal, $F$ is a 1 -formula or a 2 -formula Without loss of generality, we can assume that $F$ is a 1 -formula of the form $\theta^{1}$ for some $\theta$.

If $\vdash_{\mathscr{L}} \Gamma_{1}(2) \rightarrow \Theta_{1}(2)$ or $\vdash_{\mathscr{L}} \Gamma_{2}(2) \rightarrow \Theta_{2}(2)$ then $\underset{\mathscr{L}}{\vdash} \Gamma(2) \rightarrow \Theta(2)$.

Otherwise, $\underset{\mathscr{S}}{\vdash} \Gamma_{1}(1) \rightarrow \Theta_{1}(1)$ and $\underset{\mathscr{L}}{\vdash} \Gamma_{2}(1) \rightarrow \Theta_{2}(1)$.

Hence $\vdash_{\mathscr{S}} \Gamma_{1}(1), \Gamma_{2}(1)-\theta \rightarrow \Theta_{1}(1)-\theta, \Theta_{2}(1)$.

This means $\stackrel{f}{\mathscr{S}}_{\boldsymbol{f}} \Gamma(1) \rightarrow \Theta(1)$.

$\S 3$ Morphism logic $L^{U}=L\left(\mathscr{L}^{1}, \mathscr{L}^{2}, U\right)$.

Let $U$ be an unary predicate constant not in $\mathscr{L}, \mathscr{L}^{1}, \mathscr{L}^{2}$. From $\left\{\mathscr{L}^{1}, \mathscr{L}^{2}\right\}$ and $U$, we define the morphism logic $L^{U}=L\left(\mathscr{L}^{1}, \mathscr{L}^{2}, U\right)$ by the method in [27].

Then the set of formulas in $L^{U}$ (denoted by $F M^{U}$ ) is given by the following rules:

(1) $U(x)$ is a formula in $L^{U}$ for each $x \in F V$.

(2) $\theta^{1}, \theta^{2}$ are formulas in $L^{U}$ for each $\theta \in F M$.

(3) If $F$ is a formula in $L^{U}$, then $\neg F$ is a formula in $L^{U}$.

(4) If $K$ is a non empty countable set of formulas in $L^{U}$ such that only finitely many free variables occur in $K$, then $\wedge K, \vee K$ are formulas in $L^{U}$.

(5) If $F(x)$ is a formula in $L^{U}$ and $v$ does not appear in $F(x)$, then $(\forall v) F(v)$, $(\exists v) F(v)$ are formulas in $L^{u}$.

(6) All the formulas in $L^{U}$ are obtained from (1)-(5).

Obviously $F M^{L} \subseteq F M^{U}$.

Now we want to define the relativization of a formula in $\mathscr{L}^{1}$ by $U$. But our logic $\mathscr{L}$ is not always an usual two valued logic and it may have many unusual quantifiers.

So, we shall define the relativization by $U$ as a mapping, denoted by the same letter $U$, satisfying some conditions.

\subsection{Definition of Reiativization.}

A mapping $U$ from $F M^{1}$ to $F M^{U}$ is said to be a relativization by $U$ if $U$ is one to one and satisfies the following conditions (1)-(5):

(1) $U\left(\theta^{1}\right)$ is not a 2-formula and not of the form $U(x)$ for any $x, \theta$.

(2) $U\left(T^{1}\right)=\top^{1}, U\left(\perp^{1}\right)=\perp^{1}$ and if $U\left(\theta^{1}\right)$ is a 1 -formula, then $U(\theta)^{1}=\theta^{1}$ for any $\theta$.

(3) $\theta^{1}$ and $U\left(\theta^{1}\right)$ have the same set of free variables and 


$$
U\left(\theta^{1}\left(\begin{array}{l}
x \\
y
\end{array}\right)\right)=\left(U\left(\theta^{1}\right)\right)\left(\begin{array}{l}
x \\
y
\end{array}\right) \text { for any } \theta, x, y .
$$

(4)

$$
\begin{aligned}
& U\left(\theta^{1}\right)=\neg F \text { iff } \theta=\neg \psi \text { and } U\left(\psi^{1}\right)=F \text { for some } \psi \text {. } \\
& U\left(\theta^{1}\right)=\wedge K \text { iff } \theta=\wedge \Phi \text { and } K=\left\{U\left(\psi^{1}\right) ; \psi \in \Phi\right\} \text { for some } \Phi . \\
& U\left(\theta^{1}\right)=\vee K \text { iff } \theta=\vee \Phi \text { and } K=\left\{U\left(\psi^{1}\right) ; \psi \in \Phi\right\} \text { for some } \Phi .
\end{aligned}
$$

(5) $\quad U\left(\theta^{1}\right)=(\forall u) F(u)$ iff $\theta=(\forall v) \psi(v)$ and

$$
(\forall u) F(u)=(\forall u)\left(U(u) \supset U\left(\psi^{1}(u)\right) \text { for some } \psi .\right.
$$

$$
U\left(\theta^{1}\right)=(\exists u) F(u) \text { iff } \theta=(\exists v) \Psi(v) \text { and }
$$

$$
(\exists u) F(u)=(\exists v)\left(U(u)_{\Lambda} U\left(\psi^{1}(u)\right) \text { for some } \psi\right. \text {. }
$$

Let fix a relativization $U$ throughout this paper.

We shall write $U\left(\theta^{1}\right)$ by $\theta^{U}$ and call an $U$-formula, for each $\theta$. A quasi $U$ formula is a formula in $L^{U}$ of the form $U(x) \supset \theta^{U}$ or $U(x)_{\wedge} \theta^{U}$ for some $U$-formula $\theta^{U}$ and $x$.

Let $\Gamma \rightarrow \Theta$ be a sequent in $L^{U}$. Then by $\Gamma(U) \rightarrow \Theta(U)$, we shall denote the $\mathscr{L}$ sequent obtained from $\Gamma \rightarrow \Theta$ by deleting every formula which is neither 1-formula, $U$-formula nor quasi $U$-formula and then replacing every $\theta^{U}$ by $\theta, \theta^{1}$ by $\theta, U(x) \supset \theta^{U}$ by $\theta$ and $U(x)_{\wedge} \theta^{U}$ by $\theta$, for any $\theta$.

A formula $U(x)$ is said to be positive in $\Gamma \rightarrow \Theta$ if $U(x) \in \Theta$ or $\rightarrow U(x) \in \Gamma$ and negative in $\Gamma \rightarrow \Theta$ if $U(x) \in \Gamma$ or $\neg U(x) \in \Theta$ or $U(x)_{\wedge} \theta^{U} \in \Gamma$ or $U(x) \supset \theta^{U} \in \Theta$ for some $\theta$. Then the conditions 3.1 (1)-(5) make us possible to prove the following proposition 3.2 by using normal derivation theorem in [27].

\subsection{Proposition.}

Suppose $\Gamma, \Theta$ consist of only $U$-formulas, quasi $U$-formulas, 1-formulas; 2-formulas or formulas of the form $U(x)$ or $\rightarrow U(x)$ for some $x$.

Then

$$
\begin{aligned}
\vdash_{L^{U}} \Gamma \rightarrow \Theta & \text { implies } \\
& \text { (a) } U(x) \text { is positive and negative in } \Gamma \rightarrow \Theta \text { for some } x \\
\text { or } & \text { (b) } \vdash_{\mathscr{S}} \Gamma(U) \rightarrow \Theta(U) \\
\text { or } & \text { (C) } \vdash_{\mathscr{S}} \Gamma(2) \rightarrow \Theta(2) .
\end{aligned}
$$

Proof By the induction on a normal derivation $\mathscr{D}$ of $\Gamma \rightarrow \Theta$.

We shall treat only the cases that the last rule $R$ of $\mathscr{D}$ is one of the following rules; $(C),(\rightarrow \rightarrow),(\wedge \rightarrow),(\rightarrow \wedge),(\forall \rightarrow),(\rightarrow \forall)$. $R$ is a cut rule. Then $\mathscr{D}$ has the form 


$$
\begin{array}{cc}
\downarrow \mathscr{Q}_{1} & \downarrow \mathscr{Q}_{2} \\
R \frac{\Gamma_{1} \rightarrow \Theta_{1}}{\Gamma_{2} \rightarrow \Theta_{2}} \\
\Gamma_{1}, \Gamma_{2}-F \rightarrow \Theta_{1}-F, \Theta_{2} \\
\left(\Gamma=\Gamma_{1}, \Gamma_{2}-F ; \Theta=\Theta_{1}-F, \Theta_{2}\right)
\end{array}
$$

Notice that $F$ is a 1 -formula or a 2 -formula because $\mathscr{D}$ is normal.

By the hypotheses of induction,

$U(x)$ is positive and negative in $\Gamma_{1} \rightarrow \vartheta_{1}$ for some $x$

$$
\begin{aligned}
& \text { or } \vdash_{\mathscr{L}} \Gamma_{1}(U) \rightarrow \Theta_{1}(U) \\
& \text { or } \vdash_{\mathscr{L}} \Gamma_{1}(2) \rightarrow \Theta_{1}(2)
\end{aligned}
$$

and

$U(x)$ is positive and negative in $\Gamma_{2} \rightarrow \Theta_{2}$ for some $x$

$$
\begin{aligned}
& \text { or } \quad \vdash_{\mathscr{L}} \Gamma_{2}(U) \rightarrow \Theta_{2}(U) \\
& \text { or } \vdash_{\mathscr{L}} \Gamma_{2}(2) \rightarrow \Theta_{2}(2) .
\end{aligned}
$$

If $U(x)$ is positive and negative in $\Gamma_{1} \rightarrow \Theta_{1}$ or in $\Gamma_{2} \rightarrow \Theta_{2}$ then

$U(x)$ is positive and negative in $\Gamma \rightarrow \Theta$.

If $F$ is a 1 -formula and, $\vdash_{\mathscr{L}} \Gamma_{1}(2) \rightarrow \Theta_{1}(2)$ or $\underset{\mathscr{L}}{\vdash_{\mathscr{L}}} \Gamma_{2}(2) \rightarrow \Theta_{2}(2)$ then

$$
\vdash_{\mathscr{L}} \Gamma(2) \rightarrow \Theta(2) \quad \text { by 3.1. (1). }
$$

If $\quad F$ is a 1 -formula and, $\vdash_{\mathscr{L}} \Gamma_{1}(U) \rightarrow \Theta_{1}(U)$ and $\underset{\mathscr{L}}{\vdash} \Gamma_{2}(U) \rightarrow \Theta_{2}(U)$ then

$$
\vdash_{\mathscr{S}} \Gamma_{1}(U), \Gamma_{2}(U)-\theta \rightarrow \Theta_{1}(U)-\theta, \Theta_{2}(U) \text {, this is } \vdash_{\mathscr{S}} \Gamma(U) \rightarrow \Theta_{2}(U) \text {, }
$$

where $F=\theta^{1}$.

If $\quad F$ is a 2-formula and, $\underset{\mathscr{L}}{\vdash_{\mathscr{L}}} \Gamma_{1}(U) \rightarrow \Theta_{1}(U)$ or $\underset{\mathscr{L}}{\vdash} \Gamma_{2}(U) \rightarrow \Theta_{2}(U)$ then

$$
\vdash_{\mathscr{L}} \Gamma(U) \rightarrow \Theta(U) \text { by 3.1. (1). }
$$

If $\quad F$ is a 2 -formula and, $\underset{\mathscr{L}}{\vdash} \Gamma_{1}(2) \rightarrow \Theta_{1}(2)$ and $\underset{\mathscr{L}}{\vdash} \Gamma_{2}(2) \rightarrow \Theta_{2}(2)$ then

$$
\vdash_{\mathscr{L}} \Gamma_{1}(2), \Gamma_{2}(2)-\theta \rightarrow \Theta_{1}(2)-\theta, \Theta_{2}(2) \text {, this is } \vdash_{\mathscr{L}} \Gamma(2) \rightarrow \Theta(2),
$$

where $F=\theta^{2}$.

$R$ is $(\neg \rightarrow, F, \neg F)$. Then $\mathscr{D}$ has the form

$$
R \frac{\Gamma_{1} \rightarrow \Theta, F}{\neg F, \Gamma_{1} \rightarrow \Theta} \quad \text { where } \quad \Gamma=\neg F, \Gamma_{1}=\{\neg F\}^{\cup} \Gamma_{1} .
$$

Since $F$ is not a quasi $U$-formula, $F$ is an $U$-formula, 1-formula, 2-formula, 
or $U(x)$ for some $x$.

If $F$ is not an $U$-formula, then we can easily get the conclusion of this proposition by the hypothesis of induction.

So, we can assume that $F$ is an $U$-formula. Then by 3.1. (4), $\rightarrow F$ is also an $U$-formula of the form $F=\theta^{U}$ and $\rightarrow F=(\neg \theta)^{U}$.

By the hypothesis of induction

$$
\begin{aligned}
& U(x) \text { is positive and negative in } \Gamma_{1} \rightarrow \Theta, F \text { for some } x \\
& \text { or } \vdash_{\mathscr{S}} \Gamma_{1}(U) \rightarrow \Theta(U), \theta \\
& \text { or } \vdash_{\mathscr{L}} \Gamma_{1}(2) \rightarrow \Theta(2) .
\end{aligned}
$$

If $U(x)$ is positive and negative in $\Gamma_{1} \rightarrow \Theta, F$ then $U(x)$ is positive and negative in $\Gamma \rightarrow \Theta$ because $F \neq U(x),-U(x)$ by 3.1. (1).

If $\quad \vdash_{\mathscr{L}} \Gamma_{\mathbf{1}}(U) \rightarrow \Theta(U), \theta$ then $\stackrel{\mathscr{L}}{\mathscr{L}}_{-} \rightarrow \theta, \Gamma(U) \rightarrow \Theta_{1}(U)$.

This is $\vdash_{\mathscr{S}} \Gamma(U) \rightarrow \Theta(U)$.

If $\quad \vdash_{\mathscr{L}} \Gamma_{1}(2) \rightarrow \Theta(2)$ then $\vdash_{\mathscr{S}} \Gamma(2) \rightarrow \Theta(2)$ because $\Gamma(2)=\Gamma_{1}(2)$.

$R$ is $(\wedge \rightarrow, F, \wedge K)$. Then $\mathscr{D}$ has the form

$$
R \frac{F, \Gamma_{1} \rightarrow \Theta}{\wedge K, \Gamma_{1} \rightarrow \Theta} \quad \text { and } \Gamma=\wedge K, \Gamma_{1} \text {. }
$$

If $\wedge K$ is not a quasi $U$-formula or $U$-formula, then we can easily get the conclusion of this proposition.

If $\wedge K$ is a quasi $U$-formula, then $F$ is $U(x)$ or an $U$-formula. In either case, we can easily get the conclusion of this proposition.

If $\wedge K$ is an $U$-formula, we get the conclusion of this proposition because $F$ is an $U$-formula by 3.1 . (4).

$R$ is $(\rightarrow \wedge, K, \wedge K)$. Then $\mathscr{D}$ has the form

$$
\begin{aligned}
& \downarrow \mathscr{D}_{F} \\
& R \frac{\Gamma \rightarrow \Theta_{1}, F}{\Gamma \rightarrow \Theta_{1}, \wedge K}(F \in K)
\end{aligned}
$$$$
\text { and } \Theta=\Theta_{1}, \wedge K \text {. }
$$

If $\wedge K$ is not a quasi $U$-formula, then the conclusion is obvious.

If $\wedge K$ is a quasi $U$-formula, then $K=\left\{\theta^{U}, U(x)\right\}$ for some $x, \theta$.

Then $\mathscr{D}$ is

$$
R \frac{\stackrel{\downarrow}{\downarrow \mathscr{D}_{1}} \quad \begin{array}{l}
\downarrow \mathscr{D}_{2} \\
\Theta_{1}, U(x) \quad \Gamma \rightarrow \Theta_{1}, \theta^{U}
\end{array}}{\Gamma \rightarrow \Theta_{1}, U(x)_{\wedge} \theta^{U}}
$$$$
\text { and } \Theta=\Theta_{1}, U(x)_{\wedge} \theta^{U} \text {. }
$$ 
By the hypotheses of induction,

$U(z)$ is positive and negative in $\Gamma \rightarrow \Theta, \theta^{U}$ for some $z$

$$
\begin{aligned}
& \text { or } \quad \vdash_{\mathscr{L}} \Gamma(U) \rightarrow \Theta_{1}(U), \theta \\
& \text { or } \quad \vdash_{\mathscr{L}} \Gamma(2) \rightarrow \Theta_{1}(2) .
\end{aligned}
$$

If $U(z)$ is positive and negative in $\Gamma \rightarrow \Theta_{1}, \theta^{U}$ then $U(z)$ is also positive and negative in $\Gamma \rightarrow \Theta$.

$$
\begin{array}{ll}
\text { If } & \vdash_{\mathscr{S}} \Gamma(U) \rightarrow \Theta_{1}(U), \theta \text { then } \vdash_{\mathscr{S}} \Gamma(U) \rightarrow \Theta(U) \text { because } \Theta(U)=\Theta_{1}(U), \theta . \\
\text { If } \quad \vdash_{\mathscr{L}} \Gamma(2) \rightarrow \Theta_{1}(2) \text { then } \vdash_{\mathscr{S}} \Gamma(2) \rightarrow \Theta(2) \text { because } \Theta(2)=\Theta_{1}(2) .
\end{array}
$$

$R$ is $\left(\forall \rightarrow, F\left(\begin{array}{l}x \\ y\end{array}\right),(\forall u) F(u)\right)$. Then $D$ has the form

$$
\begin{aligned}
& \mid D_{1} \\
& R \frac{F\left(\begin{array}{l}
x \\
y
\end{array}\right), \Gamma_{1} \rightarrow \Theta}{(\forall u) F(u), \Gamma_{1} \rightarrow \Theta}, \quad \text { where } \quad \Gamma=(\forall u) F(u), \Gamma_{1} .
\end{aligned}
$$

Since $(\forall u) F(u)$ is not a quasi $U$-formula, $(\forall u) F(u)$ is an $U$-formula or a 1formula or a 2-formula.

If $(\forall u) F(u)$ is not an $U$-formula then the conclusion is obvious.

So, we can assume $(\forall u) F(u)$ is an $U$-formula.

Then by 3.1. (5), there is a $\psi(x) \in F M$ such that

$$
F(x)=U(x) \supset \psi^{U}(x),((\forall u) \psi(u))^{U}=(\forall u) F(u),
$$

By the hypothesis of induction,

$$
\begin{aligned}
& U(z) \text { is positive and negative in } F\left(\begin{array}{l}
x \\
y
\end{array}\right), \Gamma \rightarrow \Theta \text { for some } z \\
& \text { or } \underset{\mathscr{S}}{\vdash} \psi\left(\begin{array}{l}
x \\
y
\end{array}\right), \Gamma_{1}(U) \rightarrow \Theta(U) \\
& \text { or } \underset{\mathscr{S}}{\vdash} \Gamma_{1}(2) \rightarrow \Theta(2) .
\end{aligned}
$$

If $U(z)$ is positive and negative in $F\left(\begin{array}{l}x \\ y\end{array}\right), \Gamma_{1} \rightarrow \Theta$ then

$U(z)$ is positive and negative in $\Gamma_{1} \rightarrow \Theta$.

Hence $U(z)$ is positive and negative in $\Gamma \rightarrow \Theta$.

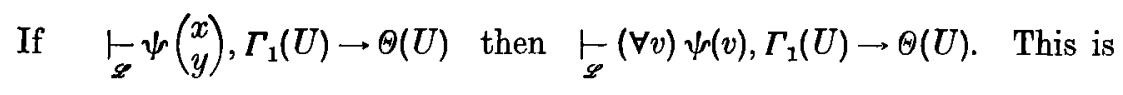

$$
\vdash_{\mathscr{L}} \Gamma(U) \rightarrow \Theta(U)
$$

If $\quad \vdash_{\mathscr{L}} \Gamma_{1}(2) \rightarrow \Theta(2)$ then $\underset{\mathscr{L}}{\vdash} \Gamma(2) \rightarrow \Theta(2)$ because $\Gamma_{1}(2)=\Gamma(2)$. 
$R$ is $(\rightarrow \forall, F(x),(\forall u) F(u))$. Then $\mathscr{D}$ has the form

$$
\begin{gathered}
\downarrow \mathscr{D}_{1} \\
R \frac{\Gamma \rightarrow \Theta_{1}, F(x)}{\Gamma \rightarrow \Theta_{1},(\forall u) F(u)}, \\
x \text { does not appear in } \Gamma, \Theta_{1} .
\end{gathered}
$$

If $(\forall u) F(u)$ is not an $U$-formula, then our conclusion is obvious.

So, we can assume that $(\forall u) F(u)$ is an $U$-formula. Then 3.1. (5)

$$
F(x)=U(x) \supset \psi^{v}(x),(\forall u) F(u)=((\forall u) \psi(u))^{U} \text { for some } \psi(x) .
$$

By the hypothesis of induction,

$$
\begin{aligned}
& U(z) \text { is positive and negative in } \Gamma \rightarrow \Theta_{1}, F(x) \text { for some } z \\
& \text { or } \vdash_{\mathscr{L}} \Gamma(U) \rightarrow \Theta_{1}(U), \psi(x) \\
& \text { or } \vdash_{\mathscr{L}} \Gamma(2) \rightarrow \Theta_{1}(2) .
\end{aligned}
$$

If $U(z)$ is positive and negative in $\Gamma \rightarrow \Theta_{1}, F(x)$ then

$U(z)$ is positive and negative in $\Gamma \rightarrow \Theta_{1}$ because $x \neq z$.

Hence $U(z)$ is positive and negative in $\Gamma \rightarrow \Theta$.

If $\vdash_{\mathscr{S}} \Gamma(U) \rightarrow \Theta_{1}(U), \psi(x)$ then $\vdash_{\mathscr{S}} \Gamma(U) \rightarrow \Theta_{1}(U),(\forall u) \psi(u)$,

because $x$ does not appear in $\Gamma(U), \Theta_{1}(U)$, by 3.1. (3).

This is $\vdash_{g} \Gamma(U) \rightarrow \Theta(U)$.

If $\quad \vdash_{\mathscr{L}} \Gamma(2) \rightarrow \Theta_{1}(2)$ then $\vdash_{\mathscr{L}} \Gamma(2) \rightarrow \Theta(2)$ because $\Theta_{1}(2)=\Theta(2)$.

3.3 Remark We can not generally hope that $\underset{\mathscr{S}}{\vdash} \boldsymbol{\phi}$ implies $\underset{L^{U}}{\vdash} \boldsymbol{\phi}^{U}$ for any $\boldsymbol{\phi} \in \boldsymbol{F} \boldsymbol{M}$.

\section{$\S 4$ Morphism logic $L^{I}=L\left(\mathscr{L}^{1}, \mathscr{L}^{2}, I_{i}\right)_{i<\omega}$.}

Let $\left\{I_{i}\right\}_{i<\omega}$ be a set of hinary predicate constants not in $\mathscr{L}, \mathscr{L}^{1}, \mathscr{L}^{2}$. From $\left\{\mathscr{L}^{1}, \mathscr{L}^{2}\right\}$ and $\left\{I_{i}\right\}_{i<\omega}$ we define the morphism logic $L^{I}=L\left(\mathscr{L}^{1}, \mathscr{L}^{2}, I_{i}\right)_{i<\omega}$ by the method in [27].

Then the set of formulas in $L^{I}$ (denoted by $F M^{I}$ ) is given by the following rules:

(1) $I_{\boldsymbol{i}}(x, y)$ is a formula in $L^{I}$ for each $i<\omega, x, y$.

(2) $\theta^{1}, \theta^{2}$ are formulas in $L^{I}$ for each $\theta \in F M$.

(3) If $F$ is a formula in $L^{I}$, then $\rightarrow F$ is a formula in $L^{I}$.

(4) If $K$ is a non empty countable set of formulas in $L^{I}$ such that only finitely many free variables occur in $K$, then $\wedge K, \vee K$ are formulas in $L^{I}$.

(5) If $F(x)$ is a formula in $L^{I}$ and $v$ does not occur in $F(x)$, then $(\forall v) F(v),(\exists v) F(v)$ are formulas in $L^{I}$

(6) All the formulas in $L^{I}$ are obtained from (1)-(5). 
Obviously $F M^{I} \subseteq F M^{I}$.

Let $\Gamma \rightarrow \Theta$ be a sequent in $L^{I}$. Then $\Gamma(1) \rightarrow \Theta(1), \Gamma(2) \rightarrow \Theta(2)$ have the same meanings as in $\S 2$.

\subsection{Proposition.}

Suppose $\Gamma$ consists of 1-formulas, 2-formulas, $I_{i}(x, y),(i<\omega, x, y)$ and $\Theta$ consists of 1-formulas, 2-formulas.

Then $\vdash_{L^{I}} \Gamma \rightarrow \Theta$ implies $\vdash_{\mathscr{S}} \Gamma(1) \rightarrow \Theta(1)$ or $\quad \vdash_{\mathscr{S}} \Gamma(2) \rightarrow \Theta(2)$.

\section{Chapter II}

\section{Interpolation theorem and characterization theorem}

In this Chapter, we shall deal with the morphism logic $L$ and prove an interpolation theorem on primitive sets and a characterization theorem on primitive sets.

All the results in this Chapter are included those of Chapter $V$ if the logic $\mathscr{L}$ is the ordinary first order logic with equality. This fact shows us the difference between Craig-Lyndon type interpolation theorems and preservation theorems in model theory i.e. the former concerns "a metamathematical identity mapping" which is independent of the existence of the equality symbol and the latter concerns "mappings" represented by predicate symbols $I_{i}, i<\omega$.

This Chapter has another purpose to help the readers to understand the contents of the following Chapters.

\section{§ 1 Interpolation problem and characterization problem.}

Let $\Psi$ be a set of sentences in $L$. Then the invarient set $I n(\Psi)$ of $\Psi$ is the set of all formulas $\theta$ in $\mathscr{L}$ such that

$\Psi \vdash_{L} \theta^{1} \supset \theta^{2}$. (This means $\vdash_{L} \Psi_{1} \rightarrow \theta^{1} \supset \theta^{2}$ for some countable subset $\Psi_{1} \subseteq \Psi$ ).

A set $\Delta \subseteq \operatorname{In}(\Psi)$ is a characterization set of $\Psi$, if for any formula $\theta$ in $\operatorname{In}(\Psi)$, there is a formula $\theta^{\prime}$ in $\Delta$ such that all the free variables in $\theta^{\prime}$ occur in $\theta$ and $\vdash_{\mathscr{Q}} \theta \equiv \theta^{\prime}$.

\subsection{Difinition.}

$\Psi$ is interpolatable if for any formulas $\phi, \psi$ in $\mathscr{L}, \Psi \vdash_{L} \boldsymbol{P}^{\mathbf{1}} \supset \psi^{2}$ implies $\vdash_{\mathscr{L}} \boldsymbol{\phi} \supset \boldsymbol{\theta}$ and $\vdash_{\mathscr{L}} \theta \supset \psi$ for some $\theta \in I n(\Psi)$ such that all the free variables in $\theta$ occur both in $\varphi$ and in $\psi$.

Notice that the latter conditions of the above definition imply the former one, i.e. $\vdash_{\mathscr{L}} \boldsymbol{\varphi} \supset \theta$ and $\vdash_{\mathscr{L}} \theta \supset \psi$ for some $\theta \in \operatorname{In}(\Psi)$ implies $\Psi \vdash_{L} \boldsymbol{\varphi}^{1} \supset \psi^{2}$ because $\vdash_{L} \boldsymbol{\varphi}^{1} \supset \theta^{1}, \vdash_{L} \theta^{2}$ $\supset \downarrow^{2}$ and $\Psi \vdash{ }_{L} \theta^{1} \supset \theta^{2}$. 
Interpolation problem "What kind of $\Psi$ is interpolatable?"

Characterization problem "For each $\Psi$, find a syntactically simple characterization set of $\Psi$."

First of all we shall state some immediate consequences of the above definitions.

\subsection{Lemma.}

If $\Delta \subseteq I n(\Psi)$ and for any $\varphi, \psi$ in $\mathscr{L}, \Psi \vdash_{L} \varphi^{\mathbf{l}} \supset \psi^{2}$ implies $\vdash_{\mathscr{L}} \boldsymbol{p} \supset \theta$ and $\vdash_{\mathscr{L}} \theta \supset \psi$ for some $\theta \in \Delta$ such that all the free variables in $\theta$ occur both in $\varphi$ and in $\psi$, then $\Psi$ is interpolatable and $\Delta$ is a characterization set of $\Psi$.

Proof Obvious from the definitions

Q.E.D.

\subsection{Proposition.}

The empty set $\phi$ is interpolatable and $\{T, \perp\}$ is a characterization set of $\phi$.

Proof It is obvious that $\{T, \perp\} \subseteq \operatorname{In}(\phi)$.

Suppose $\phi \vdash_{L} \boldsymbol{\phi}^{1} \supset \psi^{2}$. Hence $\vdash_{L} \boldsymbol{\phi}^{1} \rightarrow \psi^{2}$.

By proposition I.2.1, $\vdash_{\mathscr{L}} \neg \boldsymbol{\varphi}$ or $\vdash_{\mathscr{\mathscr { \varphi }}} \psi$.

If $\vdash_{\mathscr{L}} \rightarrow \boldsymbol{\varphi}$ then $\vdash_{\mathscr{L}} \boldsymbol{\varphi} \supset \perp$ and $\vdash_{\mathscr{L}} \perp \supset \psi$.

If $\vdash_{\mathscr{L}} \psi$ then $\vdash_{\mathscr{S}} \boldsymbol{\varphi} \supset \top$ and $\vdash_{\mathscr{L}} \top \supset \psi$.

By lemma 1.2, $\phi$ is interpolatable and $\{T, \perp\}$ is a characterization set of $\phi$.

Q.E.D.

\subsection{Proposition.}

If $\Psi$ is interpolatable and, $\Psi \vdash_{L} \varphi^{1}$ or $\Psi \vdash_{L} \phi^{2}$ then $\vdash_{\mathscr{S}} \phi$.

Proof Suppose $\Psi$ is interpolatable and $\Psi \vdash_{L} \boldsymbol{\varphi}^{\mathbf{l}}$.

Then $\Psi \vdash_{L} \rightarrow \boldsymbol{p}^{1} \rightarrow \perp^{2}$.

Since $\Psi$ is interpolatable, there is a sentence $\theta \in \operatorname{In}(\Psi)$ such that $\vdash_{\mathscr{L}} \neg \varphi \supset \theta$ and $\vdash_{\mathscr{L}} \theta \perp \perp$.

Hence we have $\vdash_{\mathscr{S}} \boldsymbol{\varphi}$.

Suppose $\Psi$ is interpolatable and $\Psi \vdash_{L} \varphi^{2}$.

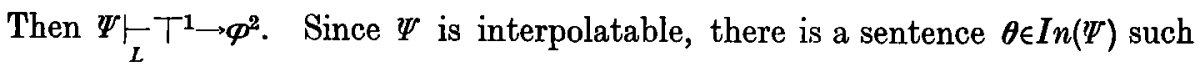
that $\vdash_{\mathscr{P}} \top \supset \theta$ and $\vdash_{\mathscr{P}} \theta \supset \boldsymbol{\theta}$.

Hence $\underset{\mathscr{L}}{\vdash_{\boldsymbol{D}}} \boldsymbol{\varphi}$.

Q.E.D. 


\subsection{Proposition.}

Let $\theta$ be $a$ sentence in $\mathscr{L}$. Then the set $\left\{\theta^{1} \supset \theta^{2}\right\}$ is interpolatable and $\{\top, \perp, \theta\}$ is a characterization set of this set.

Proof Obviously $\quad\{T, \perp, \theta\} \subseteq \operatorname{In}\left(\left\{\theta^{1} \supset \theta^{2}\right\}\right)$.

Suppose $\quad\left\{\theta^{1} \supset \theta^{2}\right\} \vdash_{L} \varphi^{1} \supset \psi^{2}$.

Then $\quad \vdash_{L} \boldsymbol{\phi}^{1}, \theta^{1} \supset \theta^{2} \rightarrow \psi^{2}$.

Hence $\quad \vdash_{L} \boldsymbol{\varphi}^{1} \rightarrow \theta^{1}, \psi^{2}$ and $\vdash_{L} \phi^{1}, \theta^{2} \rightarrow \psi^{2}$.

By proposition 1.2.1,

$\left(\vdash_{\mathscr{S}} \boldsymbol{\varphi} \supset \theta\right.$ or $\left.\vdash_{\mathscr{L}} \psi\right)$ and $\left(\vdash_{\mathscr{S}}>\boldsymbol{\varphi}\right.$ or $\left.\vdash_{\mathscr{L}} \theta \supset \psi\right)$.

If $\vdash_{\mathscr{L}} \psi$ then $\vdash_{\mathscr{L}} \boldsymbol{\varphi} \supset \top$ and $\vdash_{\mathscr{L}} T \supset \psi$.

If $\vdash_{\mathscr{L}} \neg \boldsymbol{\phi}$ then $\underset{\mathscr{L}}{\vdash} \boldsymbol{\varphi} \supset \perp$ and $\vdash_{\mathscr{S}} \perp \supset \psi$.

If "not $\vdash_{\mathscr{L}} \psi$ " and "not $\vdash_{\mathscr{L}} \neg \varphi$ ", then $\vdash_{\mathscr{L}} \boldsymbol{\varphi} \supset \boldsymbol{\theta}$ and $\vdash_{\mathscr{L}} \theta \supset \psi$.

By lemma $1.2,\left\{\theta^{1} \supset \theta^{2}\right\}$ is interpolatable and $\{T, \perp, \theta\}$ is a characterization set of $\left\{\theta^{1} \supset \theta^{2}\right\}$.

Q.E.D.

1.6 Proposition.

Let $\varphi, \psi$ be sentences in $\mathscr{L}$ such that "not $\vdash_{\mathscr{L}} \neg \varphi$ ", "not $\vdash_{\mathscr{L}} \psi$ ".

Then

$\left\{\boldsymbol{\varphi}^{1} \supset \psi^{2}\right\}$ is interpolatable iff $\vdash_{\mathscr{L}} \boldsymbol{\phi} \equiv \psi$.

PROOF By proposition 1.5, it is sufficient to prove that if $\left\{\varphi^{1} \supset \psi^{2}\right\}$ is interpolatable, then $\vdash_{\mathscr{S}} \boldsymbol{\rho} \equiv \psi$.

Suppose $\left\{\varphi^{1} \supset \psi^{2}\right\}$ is interpolatable.

Since $\left\{\varphi^{1} \supset \psi^{2}\right\} \vdash_{L} \varphi^{1} \supset \psi^{2}$, there is a sentence $\theta \in \operatorname{In}\left(\left\{\phi^{1} \supset \psi^{2}\right\}\right)$ such that $\vdash_{\mathscr{S}} \boldsymbol{\varphi} \supset \theta$ and $\underset{\mathscr{P}}{\vdash} \boldsymbol{\theta} \supset \psi$.

On the other hand, $\left\{\boldsymbol{\varphi}^{1} \supset \psi^{2}\right\} \vdash_{L} \theta^{1} \supset \theta^{2}$.

Hence $\underset{L}{\leftarrow} \theta^{1} \rightarrow \theta^{2}, \phi^{1}$ and $\underset{L}{\vdash} \theta^{1}, \psi^{2} \rightarrow \theta^{2}$.

By proposition I.2.1,

$$
\left(\vdash_{\mathscr{L}} \theta \supset \boldsymbol{\varphi} \text { or } \vdash_{\mathscr{L}} \theta\right) \text { and }\left(\vdash_{\mathscr{L}} \rightarrow \theta \text { or } \vdash_{\mathscr{L}} \psi \supset \theta\right) \text {. }
$$

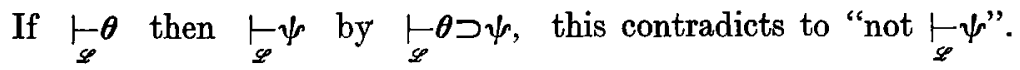

If $\vdash_{\mathscr{L}} \rightarrow \theta$ then $\vdash_{\mathscr{L}} \neg \boldsymbol{\phi}$ by $\vdash_{\mathscr{L}} \boldsymbol{\varphi} \supset \boldsymbol{\theta}$, this contradicts to "not $\vdash_{\mathscr{S}} \neg \boldsymbol{\phi}$ ". 
Hence we have $\vdash_{\mathscr{S}} \theta \supset \boldsymbol{P}$ and $\vdash_{\mathscr{S}} \psi \supset \theta$.

These mean $\vdash_{\mathscr{L}} \boldsymbol{\phi} \equiv \psi$.

Q.E.D.

By propositions 1.3-1.6, we can see that almost all the sets of sentences are not interpolatable.

At the end of this section, we shall show some important properties of $\operatorname{In}(\Psi)$, which are the source of the notion "primitive" in the next section.

\subsection{Lemma.}

In( $\Psi)$ satisfies the following conditions (1)-(5):

(1) For any formula $\theta(\vec{x}) \in F M$ whose free variables are all among $\vec{x}$ and $\vec{x}$ is a finite sequence of distinct free variables, if $(\forall \vec{u})\left(\theta^{1}(\vec{u}) \supset \theta^{2}(\vec{u})\right) \in \Psi$ then $\theta(\vec{x}) \in \operatorname{In}(\Psi)$.

(2) $\top, \perp \in \operatorname{In}(\Psi)$.

(3) Let $\vec{x}, \vec{y}$ be two sequences of free variables of the same length such that all the variables in $\vec{x}$ are distinct.

If $\theta(\vec{x}) \in \operatorname{In}(\Psi)$, then $\theta(\vec{y}) \in \operatorname{In}(\Psi)$.

(4) If $\Phi \subseteq I n(\Psi)$ is a non empty, countable set of formulas such that only finitely many free variables occur in $\Phi$, then $\wedge \Phi, \vee \Phi \in \operatorname{In}(\Psi)$.

(5) If $\theta(x) \in \operatorname{In}(\Psi)$ and $v$ does not occur in $\theta(x)$, then

$$
(\forall v) \theta(v),(\exists v) \theta(v) \in \operatorname{In}(\Psi) .
$$

Proof Obvious from the definition of invarient sets.

Q.E.D.

\section{$\S 2$ Primitive set.}

In this section, we shall define the notions "primitive sentence" and "primitive set" which is a natural extension of the sets in proposition 1.5.

Let $\theta(\vec{x})$ be a formula in $\mathscr{L}$, where $\vec{x}$ is a repetition-free enumeration of free variables in $\theta$ (i.e. all the free variables in $\vec{x}$ are distinct and the free variables in $\theta$ are exactly those in $\vec{x})$.

Then $(\forall \vec{u})\left(\theta^{1}(\vec{u}) \supset \theta^{2}(\vec{u})\right)$ is the primitive sentence defined by $\theta(\vec{x})$. A set $\Psi$ of sentences in $L$ is said to be primitive if every sentence in $\Psi$ is primitive.

Then for each primitive set $\Psi$, we define a set $\Delta(\Psi)$ as the least set satisfying the following conditions (1)-(5):

(1) If $\Psi$ has the primitive sentence defined by $\theta(\vec{x})$, then $\theta(\vec{x}) \in \Delta(\Psi)$.

(2) $T, \perp \in \Delta(\Psi)$.

(3) Let $\vec{x}, \vec{y}$ be two sequences of free variables of the same length such that all the free variables in $\vec{x}$ are distinct. If $\theta(\vec{x}) \in \Delta(\Psi)$ then $\theta(\vec{y}) \in \Delta(\Psi)$.

(4) If $\Phi$ is a non empty countable set of formulas in $\Delta(\Psi)$ such that only finitely many free variables occur in $\Phi$, then $\wedge \Phi, \vee \Phi \in \Delta(\Psi)$.

(5) If $\theta(x) \in \Delta(\Psi)$ and $v$ does not occur in $\theta(x)$, then $(\forall u) \theta(u),(\exists u) \theta(u) \in \Delta(\Psi)$. 
Notice that the above conditions (1)-(5) correspond to (1)-(5) in lemma 1.7. Hence.

\subsection{Proposition.}

$\Delta(\Psi) \subseteq \operatorname{In}(\Psi)$ for each primitive set $\Psi$.

Now we shall define $(F)$-rules for each primitive sentence $F$ to make the following arguments easy.

\subsection{Definition of $(F)$-rule.}

Let $F$ be the primitive sentence defined by $\theta(\vec{x})$, (of coruse we always assume that $\vec{x}$ is a repetition-free enumeration of free variables in $\theta$, in the cases like this).

Then an $(F)$-rule is

$$
\text { (F) } \frac{\Gamma \rightarrow \Theta}{F, \Gamma \rightarrow \Theta}
$$

where $\theta^{1}(\vec{y}) \in \Gamma$ and $\theta^{2}(\vec{y}) \in \Theta$ for some $\vec{y}$,

(of course, we always assume that $\vec{y}$ is a sequence of free variables of the same length as $\vec{x}$ ).

Let $\Psi$ be a primitive set.

A sequent $\Gamma \rightarrow \Theta$ in $L$ is said to be a $\Psi$-sequent if $\Gamma$ consists of primitive sentences in $\Psi$, 1-formulas, 2-formulas and $\Theta$ consists of 1 -formulas, 2-formulas.

Let $\Gamma \rightarrow \Theta$ be a $\Psi$-sequent. Then a $\Psi$-derivation of $\Gamma \rightarrow \Theta$ is an arrangement of $\Psi$-sequents in tree form (possibly infinite) such that

(1) the lowest sequent is $\Gamma \rightarrow \Theta$,

(2) all upper most sequents are axiom sequents,

(3) consecutive sequents in any branch of the tree are connected by one of the following rules;

(a) (w)-rule.

(b) cut rules whose cut formulas are 1-formulas or 2 -formulas and logical inference rules whose principal formulas are 1-formulas or 2-formulas.

(C) $(F)$-rules for each $F \in \Psi$.

\subsection{Lemma.}

Let $\Gamma \rightarrow \Theta$ be a $\Psi$-sequent.

If $\vdash_{L} \Gamma \rightarrow \Theta$ then there is a $\Psi$-derivation of $\Gamma \rightarrow \Theta$.

Proof This is a direct consequence of normal derivation theorem in [27].

Q.E.D.

\section{§3 Interpolation theorem and characterization theorem on primitive sets.}

In this section, we shall prove "main theorems" in this Chapter using the results in $\S 2$. 
For any primitive set $\Psi$ and any $\Psi$-derivation $\mathscr{D}$ of a $\Psi$-sequent $\Gamma \rightarrow \Theta$, we shall associate a formula $n(\mathscr{D}) \in \Delta(\Psi)$ such that
(a) $\vdash_{\mathscr{L}} \Gamma(1) \rightarrow \Theta(1), n(\mathscr{D})$,
(b) $\vdash_{\mathscr{L}} \Gamma(2), n(\mathscr{D}) \rightarrow \Theta(2)$,
(C) every free variable in $n(\mathscr{D})$ occurs both in $\Gamma(1)^{\cup} \Theta(1)$ and in $\Gamma(2)^{\cup} \Theta(2)$.

3.1 Definition of $n(\mathscr{D})$.

Case $1 \mathscr{D}$ is an axiom sequent.

If $\mathscr{D}$ is $\theta^{1} \rightarrow \theta^{1}$ or $\rightarrow \theta^{1}$, let $n(\mathscr{D})=\perp$.

If $\mathscr{D}$ is $\theta^{2} \rightarrow \theta^{2}$ or $\rightarrow \theta^{2}$ let $n(\mathscr{D})=T$.

Let $R$ be the last rule of $\mathscr{D}$.

Case $2 R$ is not $(C),(\vee \rightarrow),(\rightarrow \wedge),(\forall \rightarrow),(\rightarrow \exists),(F)$-rule for $F \in \Psi$.

Then $\mathscr{D}$ has the form $R \frac{\mathscr{D}_{1}}{\Gamma \rightarrow \Theta}$

Let $n(\mathscr{D})=n\left(\mathscr{D}_{1}\right)$.

Case $3 R$ is $(C, F)$. Then $\mathscr{D}$ has the form

$$
\begin{aligned}
& \downarrow \mathscr{D}_{1} \quad \downarrow \mathscr{D}_{2} \\
& R \frac{\Gamma_{1} \rightarrow \Theta_{1} \quad \Gamma_{2} \rightarrow \Theta_{2}}{\Gamma_{1}, \Gamma_{2}-F \rightarrow \Theta_{1}-F, \Theta}, \\
& \text { where } \quad \Gamma=\Gamma_{1}, \Gamma_{2}-F \quad \Theta=\Theta_{1}-F, \Theta_{2} \text {. }
\end{aligned}
$$

By the definition of $\Psi$-derivation, $F$ is a 1-formula or a 2-formula. If $F$ is a 1-formula of the form $\theta^{1}$, let $\vec{x}$ be the sequence of all distinct free variables which occur in $\theta$, but do not occur $\Gamma(1)^{\cup} \Theta(1)$. Let $n(\mathscr{D})=(\forall \vec{u})\left(\theta_{1}(\vec{u})^{\vee} \theta_{2}(\vec{u})\right)$, where $n\left(\mathscr{D}_{1}\right)=\theta_{1}(\vec{x})$ and $n\left(\mathscr{D}_{2}\right)=\theta_{2}(\vec{x})$.

Then by the hypotheses of induction,

$$
\begin{array}{ll}
\vdash_{\mathscr{S}} \Gamma_{1}(1) \rightarrow \Theta_{1}(1), \theta_{1}(\vec{x}), & \stackrel{\mathscr{L}}{-} \Gamma_{1}(2), \theta_{1}(\vec{x}) \rightarrow \Theta_{1}(2), \\
\vdash_{\mathscr{L}} \Gamma_{2}(1) \rightarrow \Theta_{2}(1), \theta_{2}(\vec{x}), & \stackrel{\mathscr{L}}{\vdash} \Gamma_{2}(2), \theta_{2}(\vec{x}) \rightarrow \Theta_{2}(2),
\end{array}
$$

and every free variable in $\theta_{1}(\vec{x})$ occurs both in $\Gamma_{1}(1)^{\cup} \Theta_{1}(1)$ and in $\Gamma_{1}(2)^{\cup} \Theta_{1}(2)$, every free variable in $\theta_{2}(\vec{x})$ occurs both in $\Gamma_{2}(1)^{\cup} \Theta_{2}(1)$ and in $\Gamma_{2}(2)^{\cup} \Theta_{2}(2)$.

Hence

$$
\vdash_{\mathscr{L}} \Gamma(1) \rightarrow \Theta(1), \theta_{1}(\vec{x})^{\vee} \theta_{2}(\vec{x}) \text { and }
$$

$$
\vdash_{\mathscr{S}} \Gamma(2), \theta_{1}(\vec{x})^{\vee} \theta_{2}(\vec{x}) \rightarrow \Theta(2)
$$

because

$$
\begin{aligned}
& \Gamma(1)=\Gamma_{1}(1), \Gamma_{2}(1)-\theta, \\
& \Theta(1)=\Theta_{1}(1)-\theta, \Theta_{2}(1), \\
& \Gamma(2)=\Gamma_{1}(2), \Gamma_{2}(2),
\end{aligned}
$$




$$
\Theta(2)=\Theta_{1}(2), \Theta_{2}(2)
$$

Since every variable in $\vec{x}$ does not occur in $\Gamma(1)^{\cup} \Theta(1)$,

Also

$$
\begin{aligned}
& \vdash_{\mathscr{L}} \Gamma(1) \rightarrow \Theta(1),(\forall \vec{u})\left(\theta_{1}(\vec{u})^{\vee} \theta_{2}(\vec{u})\right), \\
& \vdash_{\mathscr{L}} \Gamma(2),(\forall \vec{u})\left(\theta_{1}(\vec{u})^{\vee} \theta_{2}(\vec{u})\right) \rightarrow \Theta(2) .
\end{aligned}
$$

Since every free variable in $(\forall \vec{u})\left(\theta_{1}(\vec{u})^{\vee} \theta_{2}(\vec{u})\right)$ appear both in $\Gamma(1)^{\cup} \Theta(1)$ and in $\Gamma(2)^{\cup} \Theta(2)$, this $(\forall \vec{u})\left(\theta_{1}(\vec{u})^{\vee} \theta_{2}(\vec{u})\right)$ satisfies (a)-(C).

If $F$ is a 2-formula of the form $\theta^{2}$, let $\vec{x}$ be the sequence of all distinct free variables which occur in $\theta$ but do not occur $\Gamma(2)^{\cup} \Theta(2)$. Let $n(\mathscr{D})=(\exists \vec{u})\left(\theta_{1}(\vec{u})_{\wedge}\right.$ $\left.\theta_{2}(\vec{u})\right)$, where $n\left(\mathscr{D}_{1}\right)=\theta_{1}(\vec{x})$ and $n\left(\mathscr{D}_{2}\right)=\theta_{2}(\vec{x})$. By the method similar to the case $F$ is a l-formula, we can easily see that this $n(\mathscr{D})$ satisfies (a)-(C).

Case $4 R$ is $(\vee \rightarrow, K, \vee K)$. Then $\mathscr{D}$ has the form

$$
R \frac{\left\lfloor\mathscr{D}_{F}\right.}{\vee K, \Gamma \rightarrow \Theta(F \in K)} .
$$

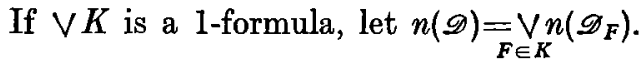

If $\vee K$ is a 2 -formula, let $n(\mathscr{D})=\underset{F \in K}{\wedge} n\left(\mathscr{D}_{F}\right)$.

Case $5 \quad R$ is $(\rightarrow \wedge, K, \wedge K)$. Similar to case 4 .

Case $6 \quad R$ is $\left(\forall \rightarrow, F\left(\begin{array}{l}x \\ y\end{array}\right),(\forall u) F(u)\right)$. Then $\mathscr{D}$ has the form

$$
R \frac{F^{\downarrow}\left(\begin{array}{l}
x \\
y
\end{array}\right), \Gamma^{\prime} \rightarrow \Theta}{(\forall v) F(v), \Gamma^{\prime} \rightarrow \Theta} .
$$

If $F\left(\begin{array}{l}x \\ y\end{array}\right)$ is a 1-formula, $y$ does not occur in $(\forall u) F(u), \Gamma^{\prime}(1), \Theta(1)$, and $n\left(\mathscr{D}_{1}\right)$ has $y$, let $n(\mathscr{D})=(\forall u) \theta(u)$, where $n\left(\mathscr{D}_{1}\right)=\theta(y)$.

If $F\left(\begin{array}{l}x \\ y\end{array}\right)$ is a 2-formula, $y$ does not occur in $(\exists u) F(u), \Gamma^{\prime}(2), \Theta(2)$, and $n\left(\mathscr{D}_{1}\right)$ has $y$, let $n(\mathscr{D})=(\exists u) \theta(u)$, where $n\left(\mathscr{D}_{1}\right)=\theta(y)$. Otherwise, let $n(\mathscr{D})=n\left(\mathscr{D}_{1}\right)$.

Case $7 \quad R$ is $\left(\rightarrow \exists, F\left(\begin{array}{l}x \\ y\end{array}\right),(\exists u) F(u)\right)$. Similar to case 6 .

Case $8 R$ is an $\left(F^{\prime}\right)$-rule, where $F$ is the primitive sentence defined by $\theta(\vec{x})$.

Then $\mathscr{D}$ has the form

$$
R \frac{\Gamma^{\prime} \rightarrow \Theta}{F, \Gamma^{\prime} \rightarrow \Theta}, \quad \text { where } \theta^{1}(\vec{y}) \in \Gamma^{\prime}, \theta^{2}(\vec{y}) \in \Theta \text { for some } \vec{y} .
$$

Let $n(\mathscr{D})=\boldsymbol{\theta}(\vec{y})$. 


\subsection{Lema.}

For any primitive set $\Psi$, any formulas $\boldsymbol{\phi}, \psi$ in $\mathscr{L}$, if $\Psi \vdash_{L} \boldsymbol{\varphi}^{1} \supset \psi^{2}$ then $\vdash_{\mathscr{L}} \boldsymbol{\varphi} \supset \boldsymbol{\theta}$ and $\vdash_{\mathscr{S}} \theta \supset \psi$ for some $\theta \in \Delta(\Psi)$ such that every free variable in $\theta$ occurs both in $\phi$ and in $\psi$. Proof Suppose $\Psi \vdash \underset{L}{ } \boldsymbol{\phi}^{1} \rightarrow \psi^{2}$.

Then for some countable set $\Psi_{1} \subseteq \Psi$

$$
\vdash_{L} \Psi_{1}, \varphi^{1} \rightarrow \psi^{2}
$$

Since $\Psi_{1}, \varphi^{1} \rightarrow \psi^{2}$ is a $\Psi$-sequent, there is a $\Psi$-derivation $\mathscr{D}$ of this sequent by lemma 2.3.

Let $n(\mathscr{D})=\theta$. Then this $\theta$ has the desired properties by (a), (b), (C). Q.E.D.

From lemma 1.2, proposition 2.1 and lemma 3.2, we have the following two theorems.

3.3 TheOREm (Interpolation theorem on primitive sets).

Every primitive set is interpolatable.

3.4 Theorem (Characterization theorem on primitive sets).

$\Delta(\Psi)$ is a characterization set of $\Psi$, for each primitive set $\Psi$.

\subsection{CoRollary.}

For any set $K$ of sentences, there is a primitive set $\Psi$ such that $\Delta(\Psi)$ is a characterization set of $K$.

Proof For $K$, let $\Psi$ be the set of primitive sentences defined by formulas in $\operatorname{In}(K)$.

Q.E.D.

\subsection{REMARK.}

Let $\mathscr{L}$ be the usual first order logic and $\mathbf{P}, \mathbf{Q}, \mathbf{R}$ are sets of predicate constants in $\mathscr{L}$. Let $\quad \Psi_{c}=\left\{(\forall \vec{u})\left(P^{1}(\vec{u}) \supset P^{2}(\vec{u})\right),(\forall \vec{u})\left(\neg P^{1}(\vec{u}) \supset \neg P^{2}(\vec{u})\right) ; P \in P\right\}$

$$
\Psi_{r}=\left\{(\forall \vec{u})\left(P^{1}(\vec{u}) \supset P^{2}(\vec{u})\right) ; P \in Q\right\} \cup\left\{(\forall \vec{u})\left(\neg P^{1}(\vec{u}) \supset \neg P^{2}(\vec{u})\right) ; P \in R\right\}
$$

Then the fact that $\Psi_{C}$ is interpolatable and $\Delta\left(\Psi_{C}\right)$ is a characterization set of $\Psi_{C}$ is essentially Craig's interpolation theorem and the fact that $\Psi$, is interpolatable and $\Delta\left(\Psi_{r}\right)$ is a characterization set of $\Psi_{r}$ is Lyndon's interpolation theorem.

\section{Chapter III}

\section{$U$-interpolation theorem and $U$-characterization theorem}

In this Chapter, we shall deal with the morphism logic $L^{U}$ and prove an $U$ interpolation theorem on $U$-primitive sets and an $U$-characterization theorem on $U$-primitive sets. 
The existence of the predicate constant $U$ makes us possible to controle universal quantifiers in the interpolating formulas, this is an advantage of $L^{U}$.

\section{\$ $U$-interpolation problem and $U$-characterization problem.}

Let $\Psi$ be a set of sentences in $L^{U}$. Then the $U$-invarient set $\operatorname{In}_{U}(\Psi)$ is the set of all formulas $\theta(\vec{x})$ in $\mathscr{L}$ such that

$$
\Psi, U(\vec{x}) \vdash_{L^{U}} \theta^{U} \supset \theta^{2},
$$

where $\vec{x}$ is a repetition-free enumeration of free variables in $\theta$ and $U(\vec{x})$ is $U\left(x_{1}\right)$ $\wedge{ }_{\wedge} U\left(x_{n}\right),\left(\vec{x}=\left\langle x_{1} \cdots x_{n}\right\rangle\right)$.

A set $\Delta \subseteq I n_{U}(\Psi)$ is an $U$-characterization set of $\Psi$ if for any formula $\theta$ in $\operatorname{In}_{U}(\Psi)$, there is a formula $\theta^{\prime}$ in $\Delta$ such that all the free variables in $\theta^{\prime}$ occur in $\theta$ and $\underset{\mathscr{S}}{+} \theta \equiv \theta^{\prime}$.

\subsection{Definition.}

$\Psi$ is $U$-interpolatable if for any $\varphi, \psi$ in $\mathscr{L}$, any finite sequence $\vec{x}$ of distinct free variables, $\quad \Psi, U(\vec{x}) \vdash_{L} \phi^{U} \supset \psi^{2}$ implies $\vdash_{\mathscr{L}} \boldsymbol{\varphi} \supset \theta$ and $\vdash_{\mathscr{L}} \theta \supset \psi$ for some $\theta \in \operatorname{In}_{U}(\Psi)$ whose free variables are among $\vec{x}$.

Notice that the latter conditions of the above definition do not generally imply the former one, e.g. let $\Psi=\left\{(\exists u)\left(U(u)_{\Lambda} P^{1}(u)\right) \supset(\exists u) P^{2}(u)\right\}, \phi=(\forall u) P(u), \psi=\theta=(\exists u) P(u)$, then $\theta \in I_{C^{\prime}}(\Psi)$ and $\vdash_{\mathscr{S}} \boldsymbol{\varphi} \supset \theta, \vdash_{\mathscr{L}} \theta \supset \psi$ but not $\Psi \vdash_{L}{ }_{U} \phi^{U} \supset \psi^{2}$ in the ordinary first order logic (cf. remark I.3.3).

On the other hand, if $\Psi$ satisfies some conditions, then we can see that the latter conditions of the above definition imply the former one, e.g. let $\Psi_{1}=\Psi^{\cup}\{(\exists u) U(u)\}$, then $\Psi_{1} \leftarrow_{L} \varphi^{U} \supset \psi^{2}$.

U-interpolation problem "What kind of $\Psi$ is $U$-interpolatable?"

U-characterization problem "For each $\Psi$, find a syntactically simple $U$ characterization set of $\Psi$."

First of all, we shall state some immediate consequences of the above definitions.

\subsection{LEMMA.}

If $\Delta \subseteq \operatorname{In}_{U}(\Psi)$ and for any $\phi, \psi$ in $\mathscr{L}$, any finite sequence of distinct free variables $\vec{x}, \Psi,\left.U(\vec{x})\right|_{L}{ }_{U} \phi^{U} \supset \psi^{2}$ implies $\vdash_{\mathscr{S}} \boldsymbol{\varphi} \supset \theta$ and $\vdash_{\mathscr{S}} \theta \supset \psi$ for some $\theta \in \Delta$ such that all the free variables in $\theta$ are among $\vec{x}$, then

$\Psi$ is $U$-interpolatable and $\Delta$ is an $U$-characterization set of $\Psi$. Proof Obvious from the definitions. 


\subsection{Proposition.}

The empty set $\phi$ is $U$-interpolatable and $\{\perp, T\}$ is an $U$-characterization set of $\phi$.

\subsection{Proposition.}

If $\Psi$ is $U$-interpolatable and, $\Psi \vdash_{L} \phi^{U}$ or $\Psi \vdash_{L} \boldsymbol{\phi}^{2}$, then $\vdash_{\mathscr{L}} \boldsymbol{\phi}$.

\subsection{Proposition.}

For each sentence $\theta$ in $\mathscr{L},\left\{\theta^{U} \supset \theta^{2}\right\}$ is $U$-interpolatable and $\{T, \perp, \theta\}$ is an $U$ characterization set of $\left\{\theta^{U} \supset \theta^{2}\right\}$.

\subsection{Propostition.}

Let $\boldsymbol{\varphi}, \Psi$ be sentences in $\mathscr{L}$ such that "not $\vdash_{\mathscr{L}} \neg \varphi$ ", "not $\vdash_{\mathscr{L}} \psi$ ".

Then

$\left\{\boldsymbol{\phi}^{U} \supset \boldsymbol{\phi}^{2}\right\}$ is $U$-interpolatable iff $\vdash_{\mathscr{L}} \boldsymbol{\phi} \equiv \psi$.

The above four propositions are similarly proved to those of II.1.3-II.1.6. by using propostition I.3.2 instead of I.2.1. By propositions 1.3-1.6 we can see that almost all the sets of sentences in $L^{U}$ are not $U$-interpolatable.

\subsection{Lemma.}

$\operatorname{In}_{U}(\Psi)$ satisfies the following conditions (1)-(5):

(1) For any formula $\theta(\vec{x}) \in F M$, where $\vec{x}$ is a repetition-free enumeration of free variables in $\theta$, if $(\forall \vec{u})\left(U(\vec{u})_{\wedge} \theta^{U}(\vec{u}) . \supset \theta^{2}(\vec{u})\right) \in \Psi$, then $\theta(\vec{x}) \in I n_{U}(\Psi)$.

(2) $\top, \perp \in \operatorname{In}_{U}(\Psi)$.

(3) Let $\vec{x}, \vec{y}$ be two sequences of free variables of the same length such that all the free variables in $\vec{x}$ are distinct.

If $\theta(\vec{x}) \in \operatorname{In}(\Psi)$ then $\theta(\vec{y}) \in \operatorname{In}_{U}(\Psi)$.

(4) If $\Phi$ is a non empty countable set of formulas in $\operatorname{In}_{C}(\Psi)$ such that only finitely many free variables occur in $\Phi$, then

$$
\wedge \Phi, \vee \Phi \in \operatorname{In}_{U}(\Psi)
$$

(5) If $\theta(\vec{x}) \in I n_{U}(\Psi)$ and $v$ does not occur in $\theta(x)$, then

$$
(\exists v) \theta(v) \in \operatorname{In}_{C}(\Psi) \text {. }
$$

Proof It is obvious that $\operatorname{In}_{U}(\Psi)$ satisfies (1)-(3).

Suppose $\Phi$ satisfies the hypotheses in (4).

Let $\vec{x}$ be a repetition-free enumeration of free variables in $\Phi$.

Since $\Phi \subseteq \operatorname{In}_{U}(\Psi)$, we have $\Psi, U(\vec{x}) \vdash_{L U} \phi^{U} \supset \phi^{2}$ for any $\phi \in \Phi$.

Hence we get

$$
\begin{aligned}
& \Psi, U(\vec{x}) \underset{L U}{\vdash} \underset{\varphi \in \Phi}{\wedge} \varphi^{U} \supset \bigwedge_{\boldsymbol{\rho} \in \Phi} \phi^{2} \\
& \Psi, U(\vec{x}) \underset{L U}{\vdash_{U}} \underset{\phi \in \Phi}{\vee} \phi^{U} \supset \underset{\phi \in \Phi}{\vee} \phi^{2} . \\
& -118-
\end{aligned}
$$$$
\text { and }
$$ 
On the other hand $\bigwedge_{\phi \in \Phi} \Phi^{U}$ is $(\wedge \Phi)^{U}, \underset{\phi \in \Phi}{\vee} \phi^{U}$ is $(\vee \Phi)^{U}$,

$$
\bigwedge_{\varphi \in \Phi} \phi^{2} \text { is }(\wedge \Phi)^{2}, \bigwedge_{\phi \in \Phi} \phi^{2} \text { is }(\vee \Phi)^{2}
$$

by I.3.1 (4).

Hence we have $\Psi, U(\vec{x}) \underset{L^{U}}{\vdash}(\wedge \Phi)^{U} \supset(\wedge \Phi)^{2}$ and $\Psi, U(\vec{x}) \underset{L}{⺊}(\vee \Phi)^{U} \supset(\vee \Phi)^{2}$.

These mean that $\wedge \Phi, \vee \Phi \in \operatorname{In}_{U}(\Psi)$.

Therefore $\operatorname{In}_{U}(\Psi)$ satisfies (4).

Suppose $\theta(x) \in I n_{U}(\Psi)$ and $v$ does not occur in $\theta(x)$.

Let $\vec{y}$ be a repetition-free enumeration of free variables except $x$ in $\theta$.

Then

$$
\begin{aligned}
& \Psi, U(\vec{y}), U(x) \underset{L^{U}}{\boldsymbol{\theta}^{U}} \theta^{U}(x) \supset \theta^{2}(x) . \\
& \therefore \Psi, U(\vec{y}) \stackrel{L}{L} U_{\mathfrak{L}} U(x)_{\wedge} \theta^{U}(x) . \supset \theta^{2}(x) . \\
& \therefore \quad \Psi, U(\vec{y}) \vdash_{L^{U}}(\exists v)\left(U(v)_{\wedge} \theta^{U}(v)\right) \supset(\exists v) \theta^{2}(v) \text {. }
\end{aligned}
$$

By I.3.1.

$$
((\exists v) \theta(v))^{U}=(\exists u)\left(U(v)_{\wedge} \theta(v)^{U}\right) .
$$

$$
\text { Hence we have }(\exists v) \theta(v) \in I n_{U}(\Psi) \text {. }
$$

Therefore $\operatorname{In}_{U}(\Psi)$ satisfies (5).

Now we call the reader's attention to the difference between lemma 1.7 in II and lemma 1.7 in III.

We want to give some sufficient conditions on $\Psi$ to make $\operatorname{In}_{\mathcal{C}}(\Psi)$ closed under universal quantifications.

Let $\phi(\vec{x}, \vec{y})$ be a formula in $\mathscr{L}, \vec{x}$ be a finite sequence of distinct free variables in $\boldsymbol{\varphi}$ and $\vec{y}$ be a repetition-free enumeration of free variables in $\phi$ except those in $\vec{x}$.

Now consider the following condition $(*)$ :

$$
\left\{\begin{array}{l}
(\forall \vec{u})(\forall \vec{v})\left(U(\vec{v})_{\Lambda} \varphi^{2}(\vec{u}, \vec{v}) \cdot \supset U(\vec{u})\right) \in \Psi \quad \text { and } \\
(\forall \vec{u})(\forall \vec{v})\left(U(\vec{u})_{\Lambda} U(\vec{v})_{\Lambda} \neg \varphi^{U}(\vec{u}, \vec{v}) . \supset \neg \varphi^{2}(\vec{u}, \vec{v})\right) \in \Psi .
\end{array}\right.
$$

Then we can easily prove that if $\Psi$ satisfies $\left(^{*}\right)$ and $\theta(\vec{x}) \in \operatorname{In}_{U}(\Psi)$, then

$$
(\forall \vec{u})(\varphi(\vec{u}, \vec{y}) \supset \theta(\vec{u})) \in I n_{U}(\Psi) \text {. }
$$

But this condition $\left(^{*}\right)$ is not appropriate to further developments in the following sections, especially in the definitions of $n(\mathscr{D})$ in $\S 3$.

So, we introduce the equality symbol $\Omega$ to $\mathscr{L}$ to get appropriate conditions on $\operatorname{In}_{U}(\Psi)$.

1.8 AdDition of the equality symbol $\bumpeq$ to $\mathscr{L}$.

We introduce the equality symbol $\bumpeq$ to $\mathscr{L}$ and add the following conditions (24, (25) to requirements on $\mathscr{L}$ at I.1.1 and (6) to conditions $U$ at I.3.1. 
For any finite sequences $\vec{x}, \vec{t}$ of the same length such that $\vec{x}$ is a sequence of distinct free variables and $\vec{t}$ is a sequence of (individual) terms in $\mathscr{L}$,

(24) $t \bumpeq t \in P F M$ for any term $t$ in $\mathscr{L}$,

(25) $\vec{x} \bumpeq \vec{t}_{\wedge} \theta(\vec{x})$. $\supset \theta(\vec{t}) \in P F M$ for any $\theta(\vec{x}) \in F M$.

(6) $\left(\vec{x} \bumpeq t_{\wedge} \theta(\vec{t}) . \supset \theta(\vec{x})\right)^{U}$ is provable in $L^{U}$ for any $\theta(\vec{x}) \in F M$.

In fact, the usual first order logic with equality satisfies the above conditions.

$$
\left(\vec{x} \bumpeq \vec{t} \text { means } x_{1} \bumpeq t_{1 \wedge} \cdots \cdots_{\wedge} x_{n} \bumpeq t_{n}\right) .
$$

\subsection{Lemma.}

Suppose $\varphi(\vec{x}, \vec{y})$ satisfies the hypotheses of the condition (*) above and

If

$$
\begin{aligned}
& \left(\forall \vec{u}_{1}\right)\left(\forall \vec{v}_{1}\right)\left(\exists \vec{u}_{2}\right)\left(U\left(\vec{v}_{1}\right)_{\wedge} \boldsymbol{\rho}^{2}\left(\vec{u}_{1}, \vec{v}_{1}\right) \cdot \supset . U\left(\vec{u}_{2}\right)_{\wedge} \vec{u}_{2} \bumpeq^{2} \vec{u}_{1}\right) \in \Psi, \\
& (\forall \vec{u})(\forall \vec{v})\left(U(\vec{u})_{\wedge} U(\vec{v})_{\wedge} \neg \boldsymbol{\varphi}^{U}(\vec{u}, \vec{v}) . \supset \neg \varphi^{2}(\vec{u}, \vec{v})\right) \in \Psi .
\end{aligned}
$$

$\theta(\vec{x}) \in \operatorname{In}_{\iota}(\Psi)$, then $(\forall \vec{u})(\varphi(\vec{u}, \vec{y}) \supset \theta(\vec{u})) \in I n_{U}(\Psi)$.

Proof Suppose the hypotheses of this lemma and $\theta(\vec{x}) \in \operatorname{In}_{U}(\Psi)$.

Let $\vec{z}$ be a repetition-free enumeration of free variables in $\theta(\vec{x})$ except those in $\vec{x}, \vec{y}$.

Let $\vec{x}_{1}$ be a sequence of distinct free variables having the same length as $\vec{x}$ and every variable in $\vec{x}_{1}$ is different form any variables in $\vec{x}, \vec{y}, \vec{z}$.

Then

$$
\Psi, U\left(\vec{x}_{1}\right), U(\vec{y}), U(\vec{z}) \vdash_{L} \theta^{U}\left(\vec{x}_{1}\right) \supset \theta^{2}\left(\vec{x}_{1}\right)
$$

and

$$
\Psi, U\left(\vec{x}_{1}\right), U(\vec{y}) \underset{L^{U}}{⺊} \neg \Phi^{U}\left(\vec{x}_{1}, \vec{y}\right) \supset \neg \varphi^{2}\left(\vec{x}_{1}, \vec{y}\right) .
$$

Hence we have

$$
\begin{aligned}
\Psi, U(\vec{y})_{\wedge} \varphi^{2}(\vec{x}, \vec{y}) . \supset . U\left(\vec{x}_{1}\right)_{\wedge} \vec{x}_{1} \bumpeq^{2} \vec{x}, U(\vec{y}), U(\vec{z}) \\
\vdash_{L^{U}} U\left(\vec{x}_{1}\right) \supset\left(\varphi^{U}\left(\vec{x}_{1}, \vec{y}\right) \supset \theta^{U}\left(\vec{x}_{1}\right)\right) \rightarrow \varphi^{2}(\vec{x}, \vec{y}) \supset \theta^{2}(\vec{x})
\end{aligned}
$$

by (24, (2) in 1.8 .

Therefore

$$
\begin{aligned}
\Psi,\left(\forall \vec{u}_{1}\right)\left(\forall \vec{v}_{1}\right)\left(\exists \vec{u}_{2}\right)\left(U\left(\vec{v}_{1}\right)_{\wedge} \rho^{2}\left(\vec{u}_{1}, \vec{v}_{1}\right) . \supset \cdot U\left(\vec{u}_{2}\right)_{\wedge} \vec{u}_{2} \bumpeq^{2} \vec{u}_{1}\right), U(\vec{y}), U(z) \\
\vdash_{L}(\forall \vec{u})\left(U(\vec{u}) \supset\left(\phi^{U}(\vec{u}, \vec{y}) \supset \theta^{U}(\vec{u})\right) \rightarrow(\forall \vec{u})\left(\varphi^{2}(\vec{u}, \vec{y}) \supset \theta^{2}(\vec{u})\right) .\right.
\end{aligned}
$$

Hence

$$
\Psi, U(\vec{y}), U(\vec{z}) \underset{L^{U}}{⺊}((\forall \vec{u})(\varphi(\vec{u}, \vec{y}) \supset \theta(\vec{u})))^{U} \supset((\forall \vec{u})(\boldsymbol{\varphi}(\vec{u}, \vec{y}) \supset \theta(\vec{u})))^{2} .
$$

This means

$$
(\forall \vec{u})(\boldsymbol{\phi}(\vec{u}, \vec{y}) \supset \theta(\vec{u})) \in I n_{U}(\Psi) .
$$

Q.E.D.

\subsection{Lemma.}

For any variable $x$, any individual constant $c$ and any formula $\theta(x)$, 


$$
\begin{gathered}
(\forall u)\left(U(u)_{\wedge}(u \bumpeq c)^{U} . \supset u \Omega^{2} c^{2}\right) \in \Psi, \quad(\exists u)\left(U(u)_{\wedge}(u \bumpeq c)^{U}\right) \in \Psi \quad \text { and } \\
\theta(x) \in \operatorname{In}_{U}(\Psi) \text { imply } \quad \theta(c) \in \operatorname{In}_{U}(\Psi) .
\end{gathered}
$$

Proof Assume

$$
\begin{aligned}
& (\forall u)\left(U(u)_{\wedge}(u \bumpeq c)^{U} . \supset u \Omega^{2} c^{2}\right) \in \Psi, \\
& (\exists u)\left(U(u)_{\wedge}(u \bumpeq c)^{U}\right) \in \Psi, \\
& \theta(x) \in \operatorname{In}_{U}(\Psi) .
\end{aligned}
$$

Let $\vec{y}$ be a repetition-free enumeration of free variables in $\theta$ except $x$ and $\boldsymbol{\theta}=\boldsymbol{\theta}(\boldsymbol{x}, \vec{y})$.

Then

$$
\begin{aligned}
& \Psi, U(x), U(\vec{y}) \vdash_{L^{U}} \theta^{U}(x, \vec{y}) \supset \theta^{2}(x, \vec{y}) . \\
& \vdash_{L}(x \bumpeq c)^{U},(\theta(c, \vec{y}))^{U} \rightarrow\left(\theta(x, \vec{y})^{U},\right. \\
& \Psi,\left.U(x)\right|_{L^{U}}(x \bumpeq c)^{U} \rightarrow x \Omega^{2} c^{2} \quad \text { and } \\
& \vdash_{L^{U}} x \bumpeq{ }^{2} c^{2}, \theta^{2}(x, \vec{y}) \rightarrow(\theta(c, \vec{y}))^{2}
\end{aligned}
$$

Since

we have

$$
\text { by the hypotheses and 1.8, (125), (6), }
$$

$$
\begin{array}{cc} 
& \Psi, U(x)_{\wedge}(x \bumpeq c)^{U}, U(\vec{y}){ }_{L^{U}}(\theta(c, \vec{y}))^{U} \supset(\theta(c, \vec{y}))^{2} . \\
\text { Hence } & \Psi,(\exists u)\left(U(u)_{\wedge}(u \bumpeq c)^{U}\right), U(\vec{y}) \vdash_{L^{U}}(\theta(c, \vec{y}))^{U} \supset(\theta(c, \vec{y}))^{2} . \\
\text { Since } & (\exists u)\left(U(u)_{\wedge}(u \bumpeq c)^{U}\right) \in \Psi \quad \text { we have } \\
& \theta(c) \in I n_{U}(\Psi) .
\end{array}
$$

Notice that $U\left(c^{1}\right), U\left(c^{2}\right), U(c)$ are not formulas in $L^{U}$.

\subsection{LEMMA.}

For any variable $x$, any $n$-ary function symbol $f$ and any formula $\theta(x)$,

$$
(\forall \vec{u})(\exists v)\left(U(\vec{u}) \supset . U(v)_{\wedge}(v \bumpeq f(\vec{u}))^{U}\right) \in \Psi,
$$

$$
(\forall \vec{u})(\forall v)\left(U(\vec{u})_{\wedge} U(v)_{\wedge}(v \bumpeq f(\vec{u}))^{U} . \supset(v \bumpeq f(\vec{u}))^{2}\right) \in \Psi
$$

and $\theta(x) \in \operatorname{In} n_{U}(\Psi)$ imply $\theta(f(\vec{y})) \in \operatorname{In} n_{U}(\Psi)$ for any finite sequence of free variables $\vec{y}$ of length $n$.

Proor Let $\vec{y}$ be a finite sequence of free variables of length $n$.

$$
\begin{aligned}
\text { Suppose } & (\forall \vec{u})(\exists v)\left(U(\vec{u}) \supset . U(v)_{\wedge}(v \bumpeq f(\vec{u}))^{U}\right) \in \Psi, \\
& (\forall \vec{u})(\forall v)\left(U(\vec{u})_{\wedge} U(v)_{\wedge}(v \bumpeq f(\vec{u}))^{U} . \supset(v \bumpeq f(\vec{u}))^{2}\right) \in \Psi, \\
& \theta(x) \in I n_{U}(\Psi) .
\end{aligned}
$$

Without loss of generality, we can assume that $x$ does not appear in $\vec{y}$.

Let $\vec{y}_{1}$ be a repetition-free enumeration of free variables in $\theta$ except $x$.

Then

$$
\Psi, U(x), U\left(\vec{y}_{1}\right) \underset{L^{U}}{\vdash} \theta^{U}\left(x, \vec{y}_{1}\right) \supset \theta^{2}\left(x, \vec{y}_{1}\right) \text {. }
$$


Since

$$
\begin{aligned}
& \vdash_{L^{U}}(x \bumpeq f(\vec{y}))^{U},\left(\theta\left(f(\vec{y}), \vec{y}_{1}\right)\right)^{U} \rightarrow\left(\theta\left(x, \vec{y}_{1}\right)\right)^{U}, \\
& \Psi, U(\vec{y}), U(x) \underset{L^{U}}{\vdash}(x \bumpeq f(\vec{y}))^{U} \rightarrow(x \bumpeq f(\vec{y}))^{2} \quad \text { and } \\
& \vdash^{U}(x \bumpeq f(\vec{y}))^{2}, \theta^{2}\left(x, \vec{y}_{1}\right) \rightarrow\left(\theta\left(f(\vec{y}), \vec{y}_{1}\right)\right)^{2}
\end{aligned}
$$

we have

by the hypotheses and 1.8. (2) (6),

$$
\Psi, U(\vec{y}) \supset . U(x\rangle_{\wedge}(x \bumpeq f(\vec{y}))^{U}, U(\vec{y}), U\left(\vec{y}_{1}\right) \leftarrow_{L^{U}}\left(\theta\left(f(\vec{y}), \vec{y}_{1}\right)\right)^{U} \supset\left(\theta\left(f(\vec{y}), \vec{y}_{1}\right)\right)^{2} .
$$

Hence

$$
\begin{aligned}
\Psi,(\forall \vec{u})(\exists v)\left(U(\vec{u}) \supset . U(v)_{\wedge}(v \bumpeq f(\vec{u}))^{U}\right), \\
\quad U(\vec{y}), U\left(\vec{y}_{1}\right) \vdash_{L^{U}}\left(\theta\left(f(\vec{y}), \vec{y}_{1}\right)\right)^{U} \supset\left(\theta\left(f(\vec{y}), \vec{y}_{1}\right)\right)^{2} .
\end{aligned}
$$

Since

$$
\begin{aligned}
& (\forall \vec{u})(\exists v)\left(U(\vec{u}) . \supset U(v)_{\wedge}\left(v \bumpeq f(\vec{u})^{U}\right) \in \Psi, \quad\right. \text { we have } \\
& \theta(f(\vec{y})) \in \operatorname{In}_{U}(\Psi) .
\end{aligned}
$$

\section{$\S 2$ U-primitive sets.}

As in $\S 2$ in the Chapter II, we shall define the notions "U-primitive sentence" and "U-primitive set".

Let $\theta(\vec{x})$ be a formula in $\mathscr{L}$ and $\vec{x}$ is a repetition-free enumeration of free variables in $\theta$.

Let $\boldsymbol{\phi}(\vec{x}, \vec{y})$ be a formula in $\mathscr{L}, \vec{x}$ is a sequence of distinct free variables in $\boldsymbol{\varphi}$ and $\vec{y}$ a repetition-free enumeration of free variables in $\boldsymbol{\varphi}$ except those in $\vec{x}, c$ be an individual constant in $\mathscr{L}, f$ be a $n$-ary function symbol in $\mathscr{L}$.

Then

$(\forall \vec{u})\left(U(\vec{u})_{\wedge} \theta^{U}(\vec{u}) . \supset \theta^{2}(\vec{u})\right)$ is the $U$-primitive sentence of type 1 defined by $\theta(\vec{x})$,

$\left(\forall \vec{u}_{1}\right)\left(\forall \vec{v}_{1}\right)\left(\exists \vec{u}_{2}\right)\left(U\left(\vec{v}_{1}\right)_{\wedge} \phi^{2}\left(\vec{u}_{1}, \vec{v}_{1}\right) \cdot \supset . U\left(\vec{u}_{2}\right)_{\wedge} \vec{u}_{2} \bumpeq^{2} \vec{u}_{1}\right)$ is the $U$-primitive sentence of type 2 defined by $(\varphi, \vec{x})$,

$(\exists u) U(u)$ is the $U$-primitive sentence of type 3 ,

$(\exists u)\left(U(u)_{\wedge}(u \bumpeq c)^{U}\right)$ is the $U$-primitive sentence of type 4 defined by $c$,

$(\forall \vec{u})(\exists v)\left(U(\vec{u}) \supset . U(v)_{\wedge}(v \bumpeq f(\vec{u}))^{U}\right)$ is the $U$-primitive sentence of type 5 defined by $f$.

A sentence $F$ is $U$-primitive if $F$ is $U$-primitive of type 1 or 2 or 3 or 4 or 5 .

A set $\Psi$ of sentences in $L^{U}$ is said to be $U$-primitive if

(1) every sentence in $\Psi$ is $U$-primitive,

(2) if $\Psi$ has the $U$-primitive sentence of type 2 defined by $(\boldsymbol{\varphi}, \vec{x})$, then $\Psi$ has the $U$-primitive sentence of type 1 defined by $\rightarrow \varphi$,

(3) if $\Psi$ has the $U$-primitive sentence of type 4 defined by $c$, then $\Psi$ has the $U$ - 
primitive sentence of type 1 defined by $x \bumpeq c$,

(4) if $\Psi$ has the $U$-primitive sentence of type 5 defined by $f$, then $\Psi$ has the $U$ primitive sentence of type 1 defined by $x \bumpeq f(\vec{y})$, (where $\vec{y}$ is a sequence of distinct free variables except $x$, of the length $n$ ).

For each $U$-primitive set $\Psi$, we define a set $\Delta_{U}(\Psi)$ by the least set satisfying the following conditions:

(1) If $\Psi$ has the $U$-primitive sentence of type 1 defined by $\theta$, then

(2) $T, \perp \in \Delta_{U}(\Psi)$.

$$
\theta \in \Delta_{U}(\Psi)
$$

(3) If $\vec{x}, \vec{y}$ are two sequences of free variables of the same length such that all the variables in $\vec{x}$ are distinct, then

$$
\theta(\vec{x}) \in \Delta_{U}(\Psi) \text { implies } \theta(\vec{y}) \in \Delta_{U}(\Psi) \text {. }
$$

(4) If $\Phi$ is a non empty countable set of formulas in $\Delta_{U}(\Psi)$ such that only finitely many free variables occur in $\Phi$, then

$$
\wedge \Phi, \vee \Phi \in \Delta_{U}(\Psi) \text {. }
$$

(5) If $\theta(x) \in \Delta_{U}(\Psi)$, then $(\exists u) \theta(u) \in \Delta_{U}(\Psi)$.

(6) If $\Psi$ has the $U$-primitive sentence of type 2 defined by $(\boldsymbol{\phi}, \vec{x})$ and $\theta(\vec{x}) \in \Delta_{U}(\Psi)$ then $(\forall \vec{u})(\boldsymbol{\phi}(\vec{u}) \supset \boldsymbol{\theta}(\vec{u})) \in \Delta_{U}(\Psi)$, where $\boldsymbol{\phi}=\boldsymbol{\phi}(\vec{x})$.

(7) If $\Psi$ has the $U$-primitive sentence of type 4 defined by $c$ and $\theta(x) \epsilon \Delta_{U}(\Psi)$ then $\theta(c) \in \Delta_{U}(\Psi)$.

(8) If $\Psi$ has the $U$-primitive sentence of type 5 defined by $f$ and $\theta(x) \epsilon \Delta_{U}(\Psi)$ then $\theta(f(\vec{y})) \epsilon \Delta_{U}(\Psi)$ for any finite sequence $\vec{y}$ of free variables of the length $n$.

Notice that the above definition of $\Delta_{U}(\Psi)$ corresponds to lemmas $1.7,1.8,1.9$, $1.10,1.11$.

Hence, we have

2.1 Proposition. $\Delta_{U}(\Psi) \subseteq \operatorname{In}_{U}(\Psi)$ for any $U$-primitive set $\Psi$.

Now as in the $\S 2$ of II, we introduce $(F)$-rules for each $U$-primitive sentence $F$.

\subsection{Definition.}

For each $U$-primitive sentence $F$, we define $(F)$-rules by the followings:

(1) If $F$ is the $U$-primitive sentence of type 1 defined by $\theta(\vec{x})$,

then

$$
\text { (F) } \frac{\Gamma \rightarrow \Theta, U\left(y_{1}\right) \cdots \cdots \Gamma \rightarrow \Theta, U\left(y_{n}\right) \quad \Gamma \rightarrow \Theta, \theta^{U}(\vec{y})}{F, \Gamma \rightarrow \Theta},
$$

(2) If $F$ is the $U$-primitive sentence of type 2 defined by $(\varphi, \vec{x})$, then 
(F) $\frac{\Gamma \rightarrow \Theta, U\left(y_{1}{ }^{\prime}\right) \cdots r \rightarrow \Theta, U\left(y_{n}{ }^{\prime}\right) U\left(x_{1}{ }^{\prime}\right), \cdots, U\left(x_{n}{ }^{\prime}\right), x_{1}{ }^{\prime} \bumpeq^{2} x_{1}{ }^{\prime \prime}, \cdots, x_{n}{ }^{\prime} \bumpeq{ }^{2} x_{n}{ }^{\prime \prime}, \Gamma \rightarrow \Theta}{F, \Gamma \rightarrow \Theta}$,

where $\boldsymbol{\phi}=\boldsymbol{\phi}(\vec{x}, \vec{y}), \vec{x}, \vec{y}$ satisfy the hypotheses of the definition of $U$-primitive sentence, $\boldsymbol{\varphi}^{2}\left(x_{1}{ }^{\prime \prime}, \cdots, x_{n}{ }^{\prime \prime}, y_{1}{ }^{\prime}, \cdots, y_{m}{ }^{\prime}\right) \epsilon \Gamma$,

$x_{1}{ }^{\prime}, \cdots, x_{n}{ }^{\prime}$ are all distinct, different from $x_{1}{ }^{\prime \prime}, \cdots, x_{m}{ }^{\prime \prime}, y_{1}{ }^{\prime}, \cdots y_{m}{ }^{\prime}$ and don't appear in $\Gamma, \Theta$.

(3) If $F$ is the $U$-primitive sentence of type 3 , then

$$
\text { (F) } \frac{U(x), \Gamma \rightarrow \Theta}{F, I \rightarrow \Theta},
$$

where $x$ does not appear in $r, \Theta$.

(4) If $F$ is the $U$-primitive sentence of type 4 define by $c$, then

$$
(F) \frac{U(x),\left(x \bumpeq^{2} c\right)^{U}, \Gamma \rightarrow \Theta}{F, \Gamma \rightarrow \Theta},
$$

where $x$ does not appear in $\Gamma, \Theta$.

(5) If $F$ is the $U$-primitive sentence of type 5 defined by $f$, then

$$
(F) \frac{U(x),(x \bumpeq f(\vec{y}))^{U}, \Gamma \rightarrow \Theta \Gamma \rightarrow \Theta, U\left(y_{1}\right) \cdots \Gamma \rightarrow \Theta, U\left(y_{n}\right)}{F, \Gamma \rightarrow \Theta},
$$

where $x$ is different from $y_{1}, \cdots, y_{n}$ and does not appear in $\Gamma, \Theta$.

In addition to the rules defined above, we require some other rules with respect to relativization.

$2.3(\forall \rightarrow)^{U},(\rightarrow \exists)^{U},(\rightarrow \forall)^{U},(\exists \rightarrow)^{U}$ rules $((U)$-rules $)$.

$$
\begin{aligned}
& \left(\forall \rightarrow, \theta\left(\begin{array}{l}
x \\
y
\end{array}\right)^{U},((\forall u) \theta(u))^{U}\right) \frac{\Gamma \rightarrow \Theta, U(y) \theta^{U}\left(\begin{array}{l}
x \\
y
\end{array}\right), \Gamma \rightarrow \Theta}{((\forall u) \theta(u))^{U}, \Gamma \rightarrow \Theta}, \\
& \left(\rightarrow \exists, \theta\left(\begin{array}{l}
x \\
y
\end{array}\right)^{U},((\exists u) \theta(u))^{U}\right) \frac{\Gamma \rightarrow \Theta, U(y) \Gamma \rightarrow \Theta, \theta^{U}\left(\begin{array}{l}
x \\
y
\end{array}\right)}{\Gamma \rightarrow \Theta,((\exists u) \theta(u))^{U}}, \\
& \left(\rightarrow \forall, \theta^{U}(x),((\forall u) \theta(u))^{U}\right) \frac{U(x), \Gamma \rightarrow \Theta, \theta^{U}(x)}{\Gamma \rightarrow \Theta,((\forall u) \theta(u))^{U}},
\end{aligned}
$$

where $x$ does not occur in the lower sequent.

$$
\left(\exists \rightarrow, \theta^{U}(x),((\exists u) \theta(u))^{U}\right) \frac{U(x), \theta^{U}(x), \Gamma \rightarrow \Theta}{((\exists u) \theta(u))^{U}, \Gamma \rightarrow \Theta},
$$

where $x$ does not occur in the lower sequent. 
Let $\Psi$ be an $U$-primitive set.

Then a sequent $\Gamma \rightarrow \Theta$ in $L^{U}$ is said to be a $\Psi$-sequent if $\Gamma$ consists of $U$ formulas, 1-formulas, 2-formulas, $U(x)$ for $x \in F V$ or $F \in \Psi$ and $\Theta$ consists of $U$ formulas, 1-formulas, 2-formulas or $U(x)$ for some $x$.

Let $\Gamma \rightarrow \Theta$ be a $\Psi$-sequent. Then a $\Psi$-derivation $\mathscr{D}$ of $\Gamma \rightarrow \Theta$ is an arrangment of $\Psi$-sequents in tree form (possibly infinite) such that

(1) the lowest sequent of $\mathscr{D}$ is $\Gamma \rightarrow \Theta$,

(2) all the upper most sequents are all axiom sequents,

(3) consecutive sequents in any branch of the tree are connected by one of the following rules;

(a) (w)-rules,

(b) cut rules whose cut formulas are 1-formulas or 2-formulas,

(C) logical inference rules except $(\forall \rightarrow)(\rightarrow \forall)(\exists \rightarrow)(\rightarrow \exists)$ whose principal formulas are $U$-formulas, 1-formulas or 2-formulas,

(d) $(\forall \rightarrow)(\rightarrow \forall)(\exists \rightarrow)(\rightarrow \exists)$ rules whose principal formulas are 1-formulas or 2-formulas,

(e) (U)-rules,

(f) $(F)$-rules for $F \in \Psi$.

Then.

\subsection{Lemma.}

For any $U$-primitive set $\Psi$ and any $\Psi$-sequent $\Gamma \rightarrow \Theta$ if ${ }_{L} \Gamma \rightarrow \Theta$, there is a $\Psi$ derivation of $\Gamma \rightarrow \Theta$.

Proof This is a direct consequence of normal derivation theorem in [27].

Q.E.D.

\section{§ $3 U$-interpolation theorem and $U$-characterization theorem on $U$-primitive sets.}

In this section, we shall prove "main theorems" in this Chapter using the results in $\S 2$.

For any $U$-primitive set $\Psi$ and any $\Psi$-derivation $\mathscr{D}$ of a $\Psi$-sequent $\Gamma \rightarrow \Theta$, we shall associate $n(\mathscr{D})$ such that $n(\mathscr{D})=x \in F V$ or $n(\mathscr{D}) \epsilon \Delta_{U}(\Psi)$ and

if $n(\mathscr{D})=x \in F V$ then $U(x) \in \Gamma_{\mathrm{n}} \Theta$, if $n(\mathscr{D})=\theta \in \Delta_{\mathcal{L}}(\Psi)$ then

(a) $\vdash_{\mathscr{S}} \Gamma(U) \rightarrow \Theta(U), \theta$,

(b) $\vdash_{\mathscr{S}} \Gamma(2), \theta \rightarrow \Theta(2)$,

(C) If $x$ occurs in $\theta$, then $U(x) \in \Gamma$. 
3.1 Definition of $n(\mathscr{D})$.

Case $1 \mathscr{D}$ is an axiom sequent.

If $\mathscr{D}$ is $U(x) \rightarrow U(x)$, let $n(\mathscr{D})=x$.

If $\mathscr{D}$ is of the form $\theta^{1} \rightarrow \theta^{1}$ or $\rightarrow \theta^{1}$ let $n(\mathscr{D})=\perp$.

If $\mathscr{D}$ is of the form $\theta^{2} \rightarrow \theta^{2}$ or $\rightarrow \theta^{2}$ let $n(\mathscr{D})=T$.

Let $R$ be the last rule of $\mathscr{D}$.

Case $2 R$ is not one of the following rules;

$(C),(\vee \rightarrow),(\rightarrow \wedge),(U)$-rules and $(F)$-rules for $F \in \Psi$.

Then $\mathscr{D}$ has the form $\quad R \frac{\mathscr{D}_{1}}{\Gamma \rightarrow \Theta}$.

Let $n(\mathscr{D})=n\left(\mathscr{D}_{1}\right)$.

Case $3 R$ is $(C, F)$. Then $\mathscr{D}$ has the form

$$
\begin{aligned}
& \downarrow \mathscr{D}_{1} \quad \mid \mathscr{D}_{2} \\
& R \frac{\Gamma_{1} \rightarrow \Theta_{1} \quad \Gamma_{2} \rightarrow \Theta_{2}}{\Gamma_{1}, \Gamma_{2}-F \rightarrow \Theta_{1}-F, \Theta_{2}} .
\end{aligned}
$$

If $n\left(\mathscr{D}_{1}\right) \in F V$, let $n(\mathscr{D})=n\left(\mathscr{D}_{\mathbf{1}}\right)$.

If $n\left(\mathscr{D}_{2}\right) \epsilon F V$, let $n(\mathscr{D})=n\left(\mathscr{D}_{2}\right)$.

So, we can assume that $n\left(\mathscr{D}_{1}\right)=\theta_{1}, \epsilon \Delta_{\mathcal{L}}(\Psi), \quad n\left(\mathscr{D}_{2}\right)=\theta_{2} \in \Delta_{U}(\Psi)$.

If $F$ is a 1 -formula, let $n(\mathscr{D})=\theta_{1}{ }^{\vee} \theta_{2}$.

If $F$ is a 2 -formula, let $n(\mathscr{D})=\theta_{1 \wedge} \theta_{2}$.

Case $4 R$ is $(\vee \rightarrow, K, \vee K)$. Then $\mathscr{D}$ has the form

$$
R \frac{\mathfrak{l}_{F}}{\vee, \Gamma \rightarrow \Theta(F \in K)} .
$$

If $n\left(\mathscr{D}_{F}\right) \in F V$ for some $F \in K$, let $n(\mathscr{D})=n\left(\mathscr{D}_{F}\right)$.

So, we can assume that $n\left(\mathscr{D}_{F}\right)=\theta_{F} \in \Delta_{U}(\Psi)$ for any $F \in K$.

If $\vee K$ is a 1 -formula or an $U$-formula, let $n(\mathscr{D})_{F \in K}=\bigvee \theta_{F}$.

If $\vee K$ is a 2 -formula, let $n(\mathscr{D})=\underset{F \in K}{\wedge} \theta_{F}$.

Case $5 \quad R$ is $(\rightarrow \wedge, K, \wedge K)$. Similar to case 4 .

Case $6 \quad R$ is $\left(\forall \rightarrow, \theta\left(\begin{array}{l}x \\ y\end{array}\right)^{U},((\forall u) \theta(u))^{U}\right)$. Then $\mathscr{D}$ has the form

$$
\begin{aligned}
& \downarrow \mathscr{D}_{1} \quad \downarrow \mathscr{D}_{2} \\
& R \frac{\Gamma \rightarrow \Theta, U(y) \quad \theta^{U}\left(\begin{array}{l}
x \\
y
\end{array}\right), \Gamma \rightarrow \Theta}{((\forall u) \theta(u))^{U}, \Gamma \rightarrow \Theta} .
\end{aligned}
$$

let $n(\mathscr{D})=n\left(\mathscr{Q}_{2}\right)$.

Case $7 \quad R$ is $(\rightarrow \exists)^{U}$. Similar to case 6 . 
Case $8 R$ is $\left(\exists \rightarrow, \theta^{U}(x),((\exists u) \theta(u))^{U}\right)$. Then $\mathscr{D}$ has the form

$$
R \frac{U(x), \theta^{U}(x), \Gamma \rightarrow \Theta}{\mathscr{D}_{1}},
$$

where $x$ does not occur in the lower sequent.

If $n\left(\mathscr{D}_{1}\right) \in F V$, let $n(\mathscr{D})=n\left(\mathscr{D}_{1}\right)$ )(notice that $n\left(\mathscr{D}_{1}\right) \neq x$ ).

If $n\left(\mathscr{D}_{1}\right)=\theta_{1}(x) \in \Delta_{U}(\Psi)$, let $n(\mathscr{D})=(\exists v) \theta_{1}(v)$.

Case $9 \quad R$ is $(\rightarrow \forall)^{U}$. Similar to case 8 .

Case 10. $R$ is an (F)-rule, where $F$ is the $U$-prinitive sentence of type 1 defined by $\theta(\vec{x}), \vec{x}=\left\langle x_{1} \cdots x_{n}\right\rangle$ is a repetition-free enumeration of free variables in $\theta$.

Then $\mathscr{D}$ has the form.

$$
R \frac{\begin{array}{l}
\downarrow \mathscr{D}_{1} \cdots \cdots, U \mathscr{D}_{n} \quad \downarrow \mathscr{Q}_{n+1} \\
\Gamma \rightarrow \Theta, U\left(y_{1}\right) \cdots \Gamma \rightarrow \Theta, U\left(y_{n}\right) \quad \Gamma \rightarrow \Theta, \theta^{U}\left(y_{1}, \cdots, y_{n}\right)
\end{array}}{F, \Gamma \rightarrow \Theta}
$$

where $\theta^{2}\left(y_{1}, \cdots, y_{n}\right) \in \theta$.

If $n\left(\mathscr{D}_{n+1}\right) \in F V$, let $n(\mathscr{D})=n\left(\mathscr{D}_{n+1}\right)$.

If $n\left(\mathscr{D}_{j}\right) \neq y_{j}$ for some $1 \leqq j \leqq n$, let $n(\mathscr{P})=n\left(\mathscr{D}_{j}\right)$.

So, we can assume that

$$
\begin{aligned}
& n\left(\mathscr{D}_{n+1}\right)=\theta_{1} \in \Delta_{U}(\Psi), \\
& n\left(\mathscr{D}_{j}\right)=y_{j}, \quad j=1, \cdots, n .
\end{aligned}
$$

Then, let $n(\mathscr{D})=\theta\left(y_{1}, \cdots, y_{n}\right)^{\vee} \theta_{1}$.

Case $11 \quad R$ is an $(F)$-rule, where $F$ is the $U$-primitive sentence of type 2 defined by $(\varphi, \vec{x}), \vec{x}=\left\langle x_{1} \cdots x_{n}\right\rangle, \vec{y}=\left\langle y_{1} \cdots y_{m}\right\rangle$ is a repetition-free enumeration of free variables in $\boldsymbol{\rho}$ except those in $\vec{x}$.

Then $\mathscr{D}$ has the form

$$
\begin{aligned}
& \downarrow \mathscr{D}_{1} \cdots \cdot \mathscr{D}_{m} \quad \mathscr{D}_{m+1} \downarrow
\end{aligned}
$$

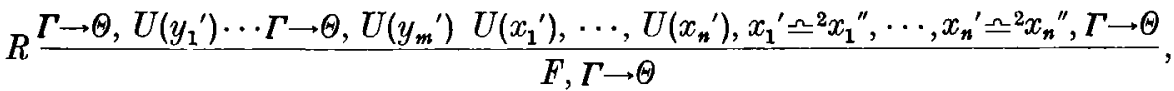

where $\varphi^{2}\left(x_{1}{ }^{\prime \prime}, \cdots, x_{n}{ }^{\prime \prime}, y_{1}{ }^{\prime}, \cdots, y_{m}{ }^{\prime}\right) \in \Gamma$,

$x_{1}{ }^{\prime}, \cdots, x_{n}{ }^{\prime}$ are all distinct, different from $x_{1}{ }^{\prime \prime}, \cdots, x_{n}{ }^{\prime \prime}, y_{1}{ }^{\prime}, \cdots, y_{m}{ }^{\prime}$ and don't appear in $\Gamma, \Theta$.

If $n\left(\mathscr{D}_{m+1}\right) \in F V \quad$ let $n(\mathscr{D})=n\left(\mathscr{D}_{m+1}\right)$.

If $n\left(\mathscr{D}_{j}\right) \neq y_{j}^{\prime}$ for some $1 \leqq j \leqq m$, let $n(\mathscr{D})=n\left(\mathscr{D}_{j}\right)$.

So, we can assume that $n\left(\mathscr{D}_{m+1}\right)=\theta\left(x_{1}{ }^{\prime}, \cdots, x_{n}{ }^{\prime}\right)$ and $n\left(\mathscr{D}_{j}\right)=y_{j}{ }^{\prime}$ for $j=1, \cdots, m$.

Let $n(\mathscr{D})=(\forall \vec{u})\left(\varphi\left(\vec{u}, y_{1}{ }^{\prime} \cdots y_{m}{ }^{\prime}\right) \supset \theta(\vec{u})\right)$.

Now we prove this $n(\mathscr{D})$ satisfies (a)-(C). 
By the hypotheses of induction

$$
\begin{aligned}
& U\left(y_{j}{ }^{\prime}\right) \in \Gamma \quad \text { for } \quad j=1, \cdots, m, \\
& \vdash_{\mathscr{L}} \Gamma(U) \rightarrow \Theta(U), \theta\left(x_{1}{ }^{\prime}, \cdots, x_{n}{ }^{\prime}\right) \\
& \vdash_{\mathscr{L}} x_{1}{ }^{\prime} \bumpeq x_{1}{ }^{\prime \prime}, \cdots, x_{n}{ }^{\prime} \bumpeq x_{n}{ }^{\prime \prime}, \Gamma(2), \theta\left(x_{1}{ }^{\prime}, \cdots, x_{n}{ }^{\prime}\right) \rightarrow \Theta(2)
\end{aligned}
$$

If $x$ appears in $\theta$, and $x \neq x_{1}{ }^{\prime}, \cdots, x_{n}{ }^{\prime}$ then $U(x) \in \Gamma$.

Hence if $x$ appear in $n(\mathscr{D})$, then $U(x) \in \Gamma$. This proves (C).

Since $\quad \Phi^{2}\left(x_{1}{ }^{\prime \prime}, \cdots, x_{n}{ }^{\prime \prime}, y_{1}{ }^{\prime}, \cdots y_{m}{ }^{\prime}\right) \in \Gamma, \vdash_{\mathscr{L}} x_{1}{ }^{\prime} \bumpeq x_{1}{ }^{\prime \prime}, \cdots, x_{n}{ }^{\prime} \bumpeq x_{n}{ }^{\prime \prime}, \Gamma(2)$,

$$
\varphi\left(x_{1}{ }^{\prime \prime}, \cdots, x_{n}{ }^{\prime \prime}\right) \supset \theta\left(x_{1}{ }^{\prime}, \cdots, x_{n}{ }^{\prime}\right) \rightarrow \Theta(2) \text {. }
$$

By replacing $\quad x_{1}{ }^{\prime}, \cdots, x_{n}{ }^{\prime}$ by $x_{1}{ }^{\prime \prime}, \cdots, x_{n}{ }^{\prime \prime}$ respectively, we have

$$
\vdash_{\mathscr{s}} \Gamma(2), \varphi\left(x_{1}^{\prime \prime}, \cdots, x_{n}{ }^{\prime \prime}\right) \supset \theta\left(x_{1}{ }^{\prime \prime}, \cdots, x_{n}{ }^{\prime \prime}\right) \rightarrow \Theta(2) .
$$

Hence we get $\quad \vdash_{\mathscr{L}} \Gamma(U) \rightarrow \Theta(U), \phi\left(x_{1}{ }^{\prime}, \cdots, x_{n}{ }^{\prime}, y_{1}{ }^{\prime}, \cdots, y_{m}{ }^{\prime}\right) \supset \theta\left(x_{1}{ }^{\prime}, \cdots, x_{n}{ }^{\prime}\right)$

$$
\text { and } \quad \vdash_{\mathscr{S}} \Gamma(2), \varphi\left(x_{1}{ }^{\prime \prime}, \cdots, x_{n}{ }^{\prime \prime}, y_{1}{ }^{\prime}, \cdots, y_{m}{ }^{\prime}\right) \supset \theta\left(x_{1}{ }^{\prime \prime}, \cdots, x_{n}{ }^{\prime \prime}\right) \rightarrow \Theta(2) \text {. }
$$

Therefore $\quad \vdash_{\mathscr{L}} \Gamma(U) \rightarrow \Theta(U), n(\mathscr{D})$ and $\vdash_{\mathscr{L}} \Gamma(2), n(\mathscr{D}) \rightarrow \Theta(2)$.

Case $12 R$ is an $(F)$-rule, where $F$ is the $U$-primitive sentence of type 3 . Then $\mathscr{D}$ has the form

$$
\begin{gathered}
\qquad \mathscr{D}_{1} \\
R \frac{U(x), \Gamma \rightarrow \Theta}{(\exists u) U(u), \Gamma \rightarrow \Theta} . \\
\text { If } n\left(\mathscr{D}_{1}\right) \in F V, \quad \text { let } n(\mathscr{D})=n\left(\mathscr{D}_{1}\right) . \\
\text { If } n\left(\mathscr{D}_{1}\right)=\theta(x) \in \Delta_{U}(\Psi), \quad \text { let } n(\mathscr{D})=(\exists u) \theta(u) .
\end{gathered}
$$

Case $13 R$ is an $(F)$-rule, where $F$ is the $U$-primitive sentence of type 4 defined by c. Then $\mathscr{D}$ has the form

$$
\begin{gathered}
\downarrow \mathscr{D}_{1} \\
R \frac{U(x),(x \bumpeq c)^{U}, \Gamma \rightarrow \Theta}{F, \Gamma \rightarrow \Theta} \\
\text { If } n(\mathscr{D}) \in F V, \quad \text { let } n(\mathscr{D})=n\left(\mathscr{D}_{1}\right) . \\
\text { If } n\left(\mathscr{D}_{1}\right)=\theta(x), \quad \text { let } n(\mathscr{D})=\theta(c) .
\end{gathered}
$$

Case $14 R$ is an $(F)$-rule, where $F$ is the $U$-primitive sentence of type 5 defined by

$f$. Then $\mathscr{D}$ has the form

$$
\begin{gathered}
\mathscr{D}_{0} \quad \downarrow \mathscr{D}_{1} \cdots \cdots \mathscr{D}_{n} \\
R \frac{U(x),(x \bumpeq f(\vec{y}))^{U}, \Gamma \rightarrow \Theta}{F, \Gamma \rightarrow \Theta} \quad \Gamma \rightarrow \Theta\left(y_{1}\right) \cdots \Gamma \rightarrow \Theta, U\left(y_{n}\right) \\
-128-
\end{gathered}
$$


If $n\left(\mathscr{D}_{0}\right) \in F V \quad$ let $n(\mathscr{D})=n\left(\mathscr{D}_{0}\right)$.

If $n\left(\mathscr{D}_{j}\right) \neq y_{j}$ for some $1 \leqq j \leqq n$, let $n(\mathscr{D})=n\left(\mathscr{D}_{j}\right)$.

So, we can assume that $n\left(\mathscr{D}_{0}\right)=\theta(x) \in \Delta_{U}(\Psi)$,

$$
\begin{array}{ll}
n\left(\mathscr{D}_{j}\right) & =y_{j}, \quad j=1, \cdots, n . \\
\text { Let } \quad & n(\mathscr{D})=\theta\left(f\left(y_{1}, \cdots, y_{n}\right)\right) .
\end{array}
$$

\subsection{Lemma.}

For any $U$-primitive set $\Psi$, any formulas $\varphi, \psi$ in $\mathscr{L}$ and any finite sequence of distinct free variables $\vec{x}$, if $\Psi, U(\vec{x}) \vdash_{L^{U}} \phi^{U} \supset \psi^{2}$ then $\vdash_{\mathscr{S}} \phi \supset \theta$ and $\vdash_{\mathscr{S}} \theta \supset \psi$ for some $\theta \epsilon$ $\Delta_{U}(\Psi)$ whose free variables are among $\vec{x}$.

Proof Suppose $\quad \Psi, U(\vec{x}) \underset{I U}{\vdash} \phi^{U} \supset \psi^{2}$.

Then for some countable $\Psi_{1} \subseteq \Psi$,

$$
\underset{L U}{\vdash} \Psi_{1}, U\left(x_{1}\right), \cdots, U\left(x_{n}\right), \phi^{U} \rightarrow \psi^{2} \quad \text { where } \quad \vec{x}=\left\langle x_{1}, \cdots, x_{n}\right\rangle .
$$

Since $\Psi_{1}, U\left(x_{1}\right), \cdots, U\left(x_{n}\right), \varphi^{U} \rightarrow \phi^{2}$ is a $\Psi$-sequent, there is a $\Psi$-derivation $\mathscr{D}$ of this sequent by lemma 2.4 .

Since $n(\mathscr{D}) \notin F V \quad n(\mathscr{D})=\theta \in \Delta_{U}(\Psi)$. Then $\vdash_{\mathscr{L}} \boldsymbol{\varphi} \supset \theta, \vdash_{\mathscr{L}} \theta \supset \psi$ and every free variable in $\theta$, is among $x_{1}, \cdots, x_{n}$.

From lemma 1.2, proposition 2.1 and lemma 3.2 , we have the following two theorems.

3.2 Theorem ( $U$-interpolation theorem on $U$-primitive sets).

Every $U$-primitive set is $U$-interpolatable.

3.4 Theorem ( $U$-characterization theorem on $U$-primitive sets).

$\Delta_{U}(\Psi)$ is an $U$-characterization set of $\Psi$, for each $U$-primitive set $\Psi$.

\subsection{Coroliary.}

For any set of sentences $K$ in $L^{U}$, there is an $U$-primitive set $\Psi$ such that $\Delta_{U}(\Psi)$ is an $U$-characterization set of $K$.

\subsection{REMARK.}

Let $\mathscr{L}$ be the usual first order logic.

Let $\Psi$, be the set of $U$-primitive sentences of type 1 defined by atomic formulas or its negations, the $U$-primitive sentence of type 3 , the $U$-primitive sentences of type 4 defined by each individual constants and the $U$-primitive sentences of type 5 defined by each function symbols. Then the fact that $\Psi_{r}$ is $U$-interpolatable and $\Delta_{U}\left(\Psi_{r}\right)$ is an $U$-characterization set of $\Psi$ is so called relativization theorem.

Let $<$ be a fixed binary predicate constant in $\mathscr{L}$ and 


$$
\Psi_{c}=\Psi_{r} \cup\left\{\left(\forall u_{1}\right)\left(\forall v_{1}\right)\left(\exists u_{2}\right)\left(u_{1}<^{2} v_{1 \wedge} U\left(v_{1}\right) \cdot \supset . U\left(u_{2}\right)_{\wedge} u_{2} \bumpeq^{2} u_{1}\right\}\right.
$$

Then the fact that $\Psi_{c}$ is $U$-interpolatable and $\Delta_{U}\left(\Psi_{\varepsilon}\right)$ is an $U$-characterization set of $\Psi_{c}$ is Feferman-Kreisel theorem on end-extension.

\section{Chapter IV}

\section{Obstruction problem of $\Psi$-extensions}

In this Chapter, we shall apply the technique developed in the proceeding Chapter to prove "obstruction problem of $\Psi$-extensions", one of whose examples is the following problem:

"Find a syntactical necessary and sufficient condition that any model of $\varphi$ have no proper end extension.

The word "obstruction" is taken from "obstruction to arithmetical extension" in A. Robinson [32].

\section{$\S 1$ Obstruction problem.}

Let $\Psi$ be a set of sentences in $L^{U}$.

Obstruction problem of $\Psi$-extension "For any formulas $\phi, \psi$ in $\mathscr{L}$ and any finite sequence of distinct free variables $\vec{x}$, find a syntactical necessary and sufficient condition in $\mathscr{L}$ that

$$
\text { “ } \Psi, U(\vec{x}) \underset{L^{U}}{\vdash} \phi^{U}, \psi^{2} \rightarrow(\forall u)(\exists v)\left(U(v)_{\wedge} u \bumpeq \Omega^{2} v\right) " \text { hold. }
$$

If $\mathscr{L}$ is the usual first order logic, $\Psi=\Psi_{e}$ in the remark 3.6 in III, and $\varphi=\psi$, then the above problem is reduced to the one stated in the introduction of this Chapter.

First of all, we should notice that if $a$ is a free variable which does not appear in $\varphi, \psi, \vec{x}$, then

$$
\begin{aligned}
& \Psi, U(\vec{x}){\underset{L}{L}} \phi^{U}, \psi^{2} \rightarrow(\forall u)(\exists v)\left(U(v)_{\wedge} u \Omega^{2} v\right) \\
\Leftrightarrow & \\
& \Psi, U(\vec{x}) \vdash_{L^{U}} \phi^{U}, \psi^{2} \rightarrow(\exists v)\left(U(v)_{\wedge} a \Omega^{2} v\right) .
\end{aligned}
$$

So, we fix a free individual variable $a$ and assume that in any derivation, $a$ is not used as an eigenvariable throughout this Chapter.

\section{$\S 2 \Delta_{U^{\alpha}}^{\alpha}(\Psi)$ sets.}

In order to define substitution, we introduce a fixed unary free predicate variable $\alpha$ not in $L^{U}$.

Then for each $U$-primitive set $\Psi$, we define the set $\Delta_{U}{ }^{\alpha}(\Psi)$ as the least set satisfying the following conditions:

(1)-8 in the definition of $\Delta_{V^{\alpha}}(\Psi)$ with a restriction that the fixed free variable $a$ does 
not appear in any formulas in $\Delta_{U}{ }^{\alpha}(\Psi)$. (9) $\alpha(x) \epsilon \Delta_{U^{\alpha}}(\Psi)$ for any $x \neq a$.

For any formula $\theta$ in $\Delta_{U}{ }^{\alpha}(\Psi)$, let $\theta^{\alpha}$ be the formula in $\mathscr{L}$ obtained from $\theta$ by replacing every $\alpha(*)$ in $\theta$ by $* \Omega *$ and $\theta_{\alpha}$ be the formula in $\mathscr{L}$ obtained from $\theta$ by replacing every $\alpha(*)$ in $\theta$ by $\neg a \bumpeq *$.

Then

\subsection{Lemma.}

If $\theta(\vec{x}) \in \Delta_{U} \alpha(\Psi)$, where $\vec{x}$ is a repetition-free enumeration of free variables in $\theta$, then

$$
\Psi, U(\vec{x}) \underset{L U}{\vdash}\left(\theta^{\alpha}\right)^{U} \rightarrow\left(\theta_{\alpha}\right)^{2},(\exists v)\left(U(v)_{\wedge} a \Omega^{2} v\right) .
$$

Proof It is sufficient to prove that the set of formulas $\theta(\vec{x})$ satisfying the conclusion of the lemma satisfies (1)-(9) in the definition of $\Delta_{U^{\alpha}}(\Psi)$.

But we can easily prove that this set satisfies (1)-(8) by the method in the proofs of lemma 1.7, lemma 1.9 in III.

So, we suffice to prove that this set saisfies (9).

Let

$$
\theta \text { be } \alpha(x),(x \neq a) \text {. }
$$

Then

$\theta^{\alpha}$ is $x \bumpeq x$ and

$$
\theta_{\alpha} \text { is } \rightarrow a \bumpeq x \text {. }
$$

But

$$
\Psi, U(x) \underset{L^{U}}{\vdash}(x \bumpeq x)^{U} \rightarrow(\neg a \bumpeq x)^{2}, U(x)_{\wedge}(a \bumpeq x)^{2} \quad \text { holds. }
$$

Hence

$$
\Psi, U(x) \underset{L U}{\vdash}\left(\theta^{\alpha}\right)^{U} \rightarrow\left(\theta_{\alpha}\right)^{2},(\exists v)\left(U(v)_{\wedge} a \Omega^{2} v\right) .
$$

$2.2(a)$-rules.

By (a)-rules, we shall mean the following type rules;

$$
\begin{gathered}
\text { (a) } \frac{\Gamma \rightarrow \Theta, U(x)}{\Gamma \rightarrow \Theta,(\exists v)\left(U(v)_{\wedge} a \Omega^{2} v\right)} \\
\text { where } a \Omega^{2} x \in \Gamma .
\end{gathered}
$$

Let $\Psi$ be an $U$-primitive set. Then a sequent $\Gamma \rightarrow \Theta$ in $L^{U}$ is $\Psi$ - $\alpha$-sequent if

$\Gamma$ consists of $U$-formulas, 1-formulas, 2-formulas, $U(x)(x \in F V)$ or $F \epsilon \Psi$ and $\Theta$ consists of $U$-formulas, 1-formulas, 2-formulas, $U(x)(x \in F V)$ or $(\exists v)\left(U(v)_{\wedge} a \Omega^{2} v\right)$.

Then a $\Psi$ - $\alpha$-derivation $\mathscr{D}$ of $\Gamma \rightarrow \Theta$ is an arrangment of $\Psi$ - $\alpha$-sequents in tree form (possibly infinite) such that

(1) the lowest sequent of $\mathscr{D}$ is $\Gamma \rightarrow \Theta$,

(2) all the uppermost sequents in $\mathscr{D}$ are all axiom sequents,

(3) consecutive sequents in any branch of the tree are connected by one of the following rules;

(a)-(f) in the definition of $\Psi$-derivation, (g) $(\alpha)$-rules.

Then by the normal derivation theorem in [27], 


\subsection{LemMa.}

For any $U$-primitive set $\Psi$ and any $\Psi$-a-sequent $\Gamma \rightarrow \Theta$ if $\vdash_{L} \Gamma \rightarrow \Theta$, then there is a $\Psi$-a-derivation of $\Gamma \rightarrow \Theta$.

\section{$\S 3$ Obstruction theorem on $\Psi$-extensions.}

In this section, we shall prove the main theorem in this Chapter.

Now, for any $U$-primitive set $\Psi$ and any $\Psi$ - $\alpha$-derivation $\mathscr{D}$ of a $\Psi$-a-sequent $\Gamma \rightarrow \Theta$, we shall associate $n(\mathscr{D})$ such that $n(\mathscr{D}) \in F V$ or $n(\mathscr{D}) \in \Delta_{U^{\alpha}}(\Psi)$ and if $n(\mathscr{D})=x \in F V$ then $U(x) \in \Gamma_{n} \Theta$, if $n(\mathscr{D})=\theta \in \Delta_{U}^{\alpha}(\Psi)$ then

$$
\begin{aligned}
& \text { (a) } \vdash_{\mathscr{L}} \Gamma(U) \rightarrow \Theta(U), \theta^{\alpha} \\
& \text { (b) } \vdash_{\mathscr{L}} \Gamma(2), \theta_{\alpha} \rightarrow \Theta(2) \\
& \text { (C) If } x \text { occurs in } \theta \text {, then } U(x) \in \Gamma \text { for any } x .
\end{aligned}
$$

3.1 Defintion of $n(\mathscr{D})$.

Case $1 \sim$ Case 12 are the same as in the case of $n(\mathscr{D})$ is $\S 3$, III.

Case 13 The last rule $R$ of $\mathscr{D}$ is an (a)-rule. Then $\mathscr{D}$ has the form

$$
\begin{array}{r}
\qquad \mathscr{D}_{1} \\
\Gamma \rightarrow \Theta, U(x) \\
\Gamma \rightarrow \Theta,(\exists v)\left(U(v)_{\wedge} a \Omega^{2} v\right) \\
\text { where } a \Omega^{2} x \in \Gamma .
\end{array}
$$

If $n(\mathscr{D}) \neq x$, let $n(\mathscr{D})=n\left(\mathscr{D}_{1}\right)$.

If $n(\mathscr{D})=x$, let $n(\mathscr{D})=\alpha(x)$.

3.2 Theorem (Obstruction theorem on $\Psi$-extension).

Let $\Psi$ be an $U$-primitive set, $\boldsymbol{\phi}, \Psi$ formulas in $\mathscr{L}, \vec{x}$ be a finite sequence of distinct free variables and if $\theta \in \Delta_{U}^{\alpha}(\Psi), \vdash_{S} \varphi \supset \theta^{\alpha}$ and all the free variables in $\theta$ are among $\vec{x}$, then $\Psi, U(\vec{x}) \underset{L^{U}}{\vdash} \Phi^{U} \rightarrow\left(\theta^{\alpha}\right)^{U}$.

Then

$$
\Psi, U(\vec{x}) \underset{L^{U}}{\vdash} \phi^{v}, \psi^{2} \rightarrow(\exists v)\left(U(v)_{\wedge} a \bumpeq^{2} v\right)
$$

$\Leftrightarrow$

$\vdash_{\mathscr{L}} \boldsymbol{\varphi} \supset \theta^{\alpha}$ and $\vdash_{\mathscr{L}} \psi \supset \neg \theta_{\alpha}$ for some $\theta \in \Delta_{U^{\alpha}}(\Psi)$ whose free variables are among $\vec{x}$.

Proof $(\Leftarrow)$ is obvious from lemma 2.1 , because $\Psi,\left.U(\vec{x})\right|_{L^{U}} \phi^{U} \rightarrow\left(\theta^{\alpha}\right)^{U}$,

$$
\underset{L U}{\vdash} \psi^{2} \rightarrow\left(\neg \theta_{\alpha}\right)^{2} \text { and } \Psi, U(\vec{x}) \underset{L^{U}}{\vdash}\left(\theta^{\alpha}\right)^{U} \rightarrow \theta_{\alpha}{ }^{2},(\exists v)\left(U(v)_{\wedge} a \bumpeq \Omega^{2} v\right) .
$$

$\Leftrightarrow$ Assume $\Psi, U(\vec{x}) \underset{L U}{\vdash} \phi^{U}, \psi^{2} \rightarrow(\exists v)\left(U(v)_{\wedge} a \Omega^{2} v\right)$. 
Then for some countable $\Psi_{1} \subseteq \Psi$

$$
\underset{L^{U}}{\vdash} \Psi_{1}, U\left(x_{1}\right), \cdots, U\left(x_{n}\right), \phi^{U}, \psi^{2} \rightarrow(\exists v)\left(U(v)_{\wedge} a \bumpeq^{2} v\right) .
$$

Since $\quad \Psi_{1}, U\left(x_{1}\right), \cdots, U\left(x_{n}\right), \phi^{U}, \psi^{2} \rightarrow(\exists v)\left(U(v)_{\wedge} a \Omega^{2} v\right)$ is a $\Psi$ - $\alpha$-sequent, by lemma 2.3 , there is a $\Psi$-a-derivation $\mathscr{D}$ of this sequent.

Then $n(\mathscr{D})$ has the desired properties.

Q.E.D.

\subsection{Example.}

Let $\mathscr{L}$ be the $L_{\omega_{1} \omega}$ with the equality symbol, an individual constant $c$ and a unary function symbol $f$.

$$
\text { Let } \begin{aligned}
& \Psi=\left\{(\forall u)\left(U(u)_{\wedge} u \bumpeq \Omega^{1} c^{1} \cdot \supset u \Omega^{2} c^{2}\right),(\forall u)(\forall v)\left(U(u)_{\Lambda} U(v)_{\wedge} u \bumpeq \Omega^{1} f^{1}(v) . \supset u \Omega^{2} f^{2}(v)\right)\right. \\
&\left.(\exists u)\left(U(u)_{\wedge} u \bumpeq \Omega^{1} c^{1}\right),(\forall u)(\exists v)\left(U(u) \supset . U(v)_{\wedge} v \bumpeq{ }^{1} f^{1}(u)\right)\right\} \\
& \varphi=(\forall u) \underset{n<\omega}{\vee}\left(u \bumpeq f^{n}(c)\right)=\psi, \quad \text { where } f^{0}(c)=c, f^{n+1}(c)=f\left(f^{n}(c)\right) .
\end{aligned}
$$

Then these $\Psi, \varphi, \psi$ satisfy the hypotheses of the above theorem and

$$
\Psi \underset{L^{U}}{\vdash} \phi^{U}, \psi^{2} \rightarrow(\exists v)\left(U(v)_{\wedge} a \bumpeq^{2} v\right) .
$$

As $\theta$, in the theorem 3.2 , we can take

$$
\theta=\bigwedge_{n<\omega}(\exists u)\left(u \bumpeq f^{n}(c)_{\wedge} \alpha(u)\right)
$$

In fact, we can state a syntactical necessary and sufficient condition that a model $\mathscr{A}$ have a proper end extension, for each countable $A$, by using the theorem 3.2 , in $L_{\omega_{1} \omega}$.

But it seems to be difficult that we can get the results, already obtained by many authors such as MacDowell-Specker, [21], Keisler-Morley [15], directly from theorem 3.2, although the present author believe that this approach along our theorem 3.2 may turn out to be useful in solving end extension problems, omitting type problems. (cf. Morley [28]).

Finally we shall show a consequence of theorem 3.2 on theories categorical at power $s_{1}$.

\subsection{Proposition.}

Let $\mathscr{L}$ be a finitary countable first order logic with equality and $T$ be a theory in $\mathscr{L}$, which is categorical at power $\mathbf{\aleph}_{1}$.

Then for any formula $\varphi(x)$ whose free variable is $x$ only, there is a sentence $\theta \in \Delta_{U}^{\alpha}\left(\Psi_{q}\right)$ such that

$$
T \vdash_{\mathscr{L}} \theta^{\alpha} \text { and } T \vdash_{\mathscr{L}} \rightarrow \theta_{\alpha}
$$

where $\Psi_{\phi}$ is the set of the following U-primitive sentences; U-primitive sentences of type 1 defined by arbitrary formulas,

$U$-primitive sentence of type 2 defined by $(\varphi, x)$, 
$U$-primitive sentence of type 3 ,

$U$-primitive sentences of type 4 defined by each individual constants,

$U$-primitive sentences of type 5 defined by each function symbols.

Proof Since $\aleph_{1}$-categorical theories do not have models $\mathscr{A}, \mathscr{B}$ such that $\mathscr{B}$ is a

proper elementary extension of $\mathscr{A}$ which preserives $\boldsymbol{\phi}$,

(cf. [29]).

we have $\quad \Psi_{\varphi} \leftarrow T^{U} T^{U}, T^{2} \rightarrow(\exists v)\left(U(v)_{\wedge} a \Omega^{2} v\right)$

Q.E.D.

\section{Chapter V}

\section{$I$-interpolation theorem and $I$-characterization theorem}

In this Chapter, we shall deal with the morphism logic $L^{I}$ and prove an $I$ interpolation theorem on $I$-primitive sets and an $I$-characterization theorem on $I$-primitive sets.

The existence of predicate constants $\left\{I_{i}\right\} i<\omega$ make us possible to controle quantifiers in the interpolating formulas. In particular the existence of infinitely many predicate constants $\left\{I_{i}\right\} i<\omega$ make us possible to controle some hierachy of quantification in the interpolating formulas (cf. $\forall \exists$-class).

As stated in the introduction of this paper, the contents of this Chapter are very different from those in the proceeding Chapters because in this Chapter we shall consider "morphisms" expressed by predicate constants $I_{i}, i<\omega$, on the other hand, in the proceeding Chapters, we only considered the morphism expressed by the metamathematical identity relation $x \rightarrow x$ on the set of free variables, these are typically seen in the conditions on free variables in the interpolating formulas in II.1.1 and III.1.1.

\section{$\S 1 I$-interpolation problem and $I$-characterization problem.}

Before formulating our problems, let divide the set $F V$ into mutually disjoint infinite sets $\left\{V_{i}\right\}_{i<\omega}$, and by $w^{i}$ (with or without suffixes) we shall denote a variable in $V_{i}$.

This classification of free variables will be used to indicate the correspondence between free variables and $I_{i}, i<\omega$.

Let $\Psi$ be a set of sentences in $L^{I}$.

Then, the $I$-invarient set $\operatorname{In}_{I}(\Psi)$ of $\Psi$ is the set of all formulas $\theta\left(w_{1}{ }^{i_{1}}, \cdots, w_{k}{ }^{i_{k}}\right)$ such that $w_{1}{ }^{i_{1}}, \cdots, w_{k}{ }^{i_{k}}$ is a repetition-free enumeration of free variables in $\theta$ and

$$
\begin{aligned}
\Psi \underset{L^{I}}{\vdash}\left(\forall u_{1}\right) \cdots\left(\forall u_{k}\right)\left(\forall v_{1}\right) & \cdots\left(\forall v_{k}\right)\left(I_{i_{1}}\left(u_{1}, v_{1}\right)_{\wedge} \cdots\right. \\
& \left.\cdots{ }_{\wedge} I_{i_{k}}\left(u_{k}, v_{k}\right)_{\wedge} \theta^{1}\left(u_{1} \cdots u_{k}\right) . \supset \theta^{2}\left(v_{1} \cdots v_{k}\right)\right) .
\end{aligned}
$$

A set $\Delta \subseteq I n_{I}(\Psi)$ is an $I$-characterization set of $\Psi$ if for any formula $\theta \in I n_{I}(\Psi)$, there is a formula $\theta^{\prime} \in \Delta$ such that $\vdash_{\mathscr{L}} \theta \equiv \theta^{\prime}$ and every free variable in $\theta^{\prime}$ appears in $\theta$. 


\subsection{Definition.}

$\Psi$ is $I$-interpolatable if for any distinct free variables $w_{1}{ }^{i_{1}}, \cdots, w_{k}{ }^{i_{k}}$, any formulas $\boldsymbol{\varphi}\left(w_{1}^{i_{1}}, \cdots, w_{k}{ }^{i_{k}}\right), \psi\left(w_{1}^{i_{1}}, \cdots, w_{k}^{i_{k}}\right)$,

$$
\begin{aligned}
\Psi \underset{L^{I}}{\vdash}\left(\forall u_{1}\right) \cdots\left(\forall u_{k}\right)\left(\forall v_{1}\right) & \cdots\left(\forall v_{k}\right)\left(I_{i_{1}}\left(u_{1}, v_{1}\right)_{\wedge} \cdots\right. \\
& \left.\cdots{ }_{\Lambda} I_{i_{k}}\left(u_{k}, v_{k}\right)_{\wedge} \phi^{1}\left(u_{1} \cdots u_{k}\right) . \supset \psi^{2}\left(v_{1} \cdots v_{k}\right)\right)
\end{aligned}
$$

implies

$\vdash_{\mathscr{L}} \boldsymbol{\varphi} \supset \theta$ and $\vdash_{\mathscr{L}} \theta \supset \psi$ for some $\theta \in \operatorname{In}_{I}(\Psi)$ whose free variables are among $w_{1}^{i_{1}}, \cdots, w_{k}^{i_{k}}$.

Notice that the latter conditions of the above definition imply the former one,

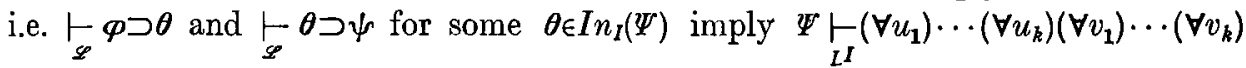
$\left(I_{i_{1}}\left(u_{1}, v_{1}\right)_{\wedge} \cdots{ }_{\wedge} I_{i_{k}}\left(u_{k}, v_{k}\right)_{\wedge} \phi^{1}\left(u_{1}, \cdots, u_{k}\right) \cdot \supset \psi^{2}\left(v_{1}, \cdots, v_{k}\right)\right) \quad$ because $\quad \underset{L I}{\vdash} \phi^{1} \supset \theta^{1}$, $\underset{L I}{\vdash} \theta^{2} \supset \psi^{2}$ and $\underset{L I}{\vdash}\left(\forall u_{1}\right) \cdots\left(\forall u_{k}\right)\left(\forall v_{1}\right) \cdots\left(\forall v_{k}\right)\left(I_{i_{1}}\left(u_{1}, v_{1}\right)_{\wedge} \cdots{ }_{\wedge} I_{i_{k}}\left(u_{k}, v_{k}\right)_{\wedge} \theta^{1}\left(u_{1}, \cdots, u_{k}\right)\right.$. $\left.\supset \theta^{2}\left(v_{1}, \cdots, v_{k}\right)\right)$.

I-interpolation problem "What kind of $\Psi$ is $I$-interpolatable?"

$I$-characterization problem "For each $\Psi$, find a syntactically simple $I$-characterization set of $\Psi$."

As in the cases of interpolation problem, $U$-interpolation problem, first of all, we shall state some immediate consequences of the above definitions.

In the followings, $\vec{x}, \vec{y}, \vec{x}_{1}, \vec{y}_{1}, \vec{u}, \vec{u}_{1}, \vec{v}, \vec{v}_{1}, \vec{i}, \vec{j}, \vec{k}$ mean finite sequences of each symbols with suffixes, for example $\vec{x}$ means $\left\langle x_{1}, \cdots, x_{n}\right\rangle$ and $\vec{x}_{1}$ means $\left\langle x_{11}, \cdots, x_{1 n}\right\rangle$. If two sequences, e.g. $\vec{x}$ and $\vec{u}$, appear in one context, then we shall always assume every condition that is necessary to make the expression containing them meaningful.

By $\vec{w}^{i}$, we shall mean $\left\langle w_{1}^{i}, \cdots, w_{k}^{i}\right\rangle$ and by $w^{i}$, we shall mean $\left\langle w_{1}^{i_{1}}, \cdots, w_{k}^{i_{k}}\right\rangle$.

$$
\begin{array}{lll}
I_{i}(\vec{u}, \vec{v}) & \text { means } & I_{i}\left(u_{1}, v_{1}\right)_{\wedge} \cdots{ }_{\wedge} I_{i}\left(u_{k}, v_{k}\right) . \\
I_{i}(\vec{u}, \vec{v}) & \text { means } & I_{i_{1}}\left(u_{1}, v_{1}\right)_{\wedge} \cdots{ }_{\wedge} I_{i_{k}}\left(u_{k}, v_{k}\right) .
\end{array}
$$

Similarly $w_{1}^{i_{1}}, w_{2}^{i_{3}}, \cdots$ will be used, e.g. $w_{1}^{i_{1}}$ means $\left\langle w_{11}^{i_{11}}, \cdots, w_{1_{1}}^{i_{1 k_{1}}}\right\rangle$. We say that $\vec{x}, \vec{y}$ are disjoint if $\vec{x}, \vec{y}$ are disjoint as sets.

\subsection{Lemma.}

If $\Delta \subseteq \operatorname{In}_{I}(\Psi)$ and for any $w^{i}$, where all the variables in $w^{i}$ are distinct, any formulas $\varphi\left(w^{i}\right), \Psi\left(w^{i}\right)$ in $\mathscr{L}$

$$
\Psi \vdash_{L I}(\forall \vec{u})(\forall \vec{v})\left(I_{\vec{\imath}}(\vec{u}, \vec{v})_{\wedge} \varphi^{1}(\vec{u}) . \supset \psi^{2}(\vec{v})\right) \quad \text { implies } \vdash_{\mathscr{L}} \boldsymbol{\varphi} \supset \boldsymbol{\theta}
$$

and $\vdash \theta \supset \psi$ for some $\theta \in \Delta$ whose free variables are among $w^{i}$, then

$\Psi$ is I-interpolatable and $\Delta$ is an I-characterization set of $\Psi$.

Proof Obvious from the definitions.

Q.E.D. 
1.3 Proposition.

The empty set $\phi$ is I-interpolatable and $[T, \perp\}$ is an I-characterization set of $\phi$.

\subsection{Proposition.}

If $\Psi$ is I-interpolatable and $\Psi \underset{L I}{\vdash} \phi^{1}$ or $\Psi \vdash_{L I} \phi^{2}$ then $\vdash_{\mathscr{S}} \phi$.

\subsection{Proposition.}

For any sentence $\theta$ in $\mathscr{L},\left\{\theta^{1} \supset \theta^{2}\right\}$ is I-interpolatable and $\{T, \perp, \theta\}$ is an 1 characterization set of $\left\{\boldsymbol{\theta}^{1} \supset \boldsymbol{\theta}^{2}\right\}$.

\subsection{Proposition.}

Let $\boldsymbol{\varphi}, \Psi$ be sentences in $\mathscr{L}$ such that "not $\vdash_{\mathscr{L}} \rightarrow \boldsymbol{\phi}$ ", "not $\vdash_{\mathscr{L}} \psi$ ".

Then

$\left\{\boldsymbol{\varphi}^{1} \supset \psi^{2}\right\}$ is I-interpolatable iff $\vdash_{\mathscr{L}} \boldsymbol{\varphi} \equiv \psi$.

The above four propositions are similarly proved to those of II.1.3, II.1.6 by using I.4.1 instead of I.2.1.

By proposition 1.3-1.6, we can see that almost all the sets of sentences in $L^{I}$ are not $I$-interpolatable.

\subsection{Lemma.}

$I n_{I}(\Psi)$ satisfies the following conditions (1)-(4):

(1) For any formula $\theta\left(w^{i}\right) \in F M$ where $w^{i}$ is a repetition-free enumeration of free variables in $\theta$ if

then

$$
\begin{gathered}
(\forall \vec{u})(\forall \vec{v})\left(I_{i}(\vec{u}, \vec{v})_{\wedge} \theta^{1}(\vec{u}) \cdot \supset \theta^{2}(\vec{v})\right) \in \Psi \\
\theta\left(w^{\hat{i}}\right) \in \operatorname{In}_{I}(\Psi) .
\end{gathered}
$$

(2) $\top, \perp \in I n_{I}(\Psi)$.

(3) Let $\vec{x}, \vec{y}$ be two sequences of free variables of the same length such that all the variables in $\vec{x}$ are distinct and $x_{l}, y_{l}$ belong to same set $V_{i}(i<\omega)$ for each $l=1, \cdots, n$. If $\theta(\vec{x}) \in I n_{I}(\Psi)$, then $\theta(\vec{y}) \in I n_{I}(\Psi)$.

(4) If $\Phi$ is a non empty countable set of formulas in $I_{I}(\Psi)$ such that only finitely many free variables occur in $\Phi$, then $\wedge \Phi, \vee \Phi \in I n_{I}(\Psi)$.

Proof Obvious from the definition of $I n_{I}(\Psi)$.

Q.E.D.

\subsection{Lemma.}

Let $w^{t}$ be a finite sequence of distinct free variables.

(i) If $(\forall \vec{u})(\exists \vec{v}) I_{i}(\vec{u}, \vec{v}) \in \Psi$ and $\theta\left(w^{i}\right) \in \operatorname{In}_{I}(\Psi)$, then $(\exists \vec{u}) \theta(\vec{u}) \in \operatorname{In}_{I}(\Psi)$.

(ii) If $(\forall \vec{v})(\exists \vec{u}) I_{i}(\vec{u}, \vec{v}) \in \Psi$ and $\theta\left(w^{i}\right) \in \operatorname{In}_{I}(\Psi)$, then $(\forall \vec{u}) \theta(\vec{u}) \in \operatorname{In}_{I}(\Psi)$. 
Proof We shall prove (i) only because (ii) can be proved similarly.

Suppose $(\forall \vec{u})(\exists \vec{u}) I_{i}(\vec{u}, \vec{v}) \epsilon \Psi$ and $\theta\left(w^{\vec{i}}\right) \epsilon \operatorname{In} I(\Psi)$.

Let $w^{i_{1}}$ be a repetition-free enumeration of free variables in $\theta$ except those in $w^{i}$ and $\vec{x}, \vec{x}_{1}, \vec{y}, \vec{y}_{1}$ be finite sequences of distinct free variables such that all the free variables appearing in one of $\vec{x}, \vec{x}_{1}, \vec{y}, \vec{y}_{1}$ are distinct and $\vec{x}, \vec{y}, w^{i}$ have the same length, $\vec{x}_{1}, \vec{y}_{1}, w_{1}{ }^{i}$ have the same length.

Since $\quad \theta\left(w^{i}, w_{1}^{i} i_{1}\right) \in \operatorname{In}(\Psi)$,

$$
\Psi \underset{L I}{\vdash}(\forall \vec{u})\left(\forall \vec{u}_{1}\right)(\forall \vec{v})\left(\forall \vec{v}_{1}\right)\left(I_{i}(\vec{u}, \vec{v})_{\wedge} I_{i_{1}}\left(\vec{u}_{1}, \vec{v}_{1}\right)_{\Lambda} \theta^{2}\left(\vec{u}, \vec{u}_{1}\right) . \supset \theta^{2}\left(\vec{v}, \vec{v}_{1}\right)\right) .
$$

Hence $\quad \Psi \vdash_{L^{I}} I_{i}(\vec{x}, \vec{y}), I_{i_{1}}\left(\vec{x}_{1}, \vec{y}_{1}\right), \theta^{1}\left(\vec{x}, \vec{x}_{1}\right) \rightarrow \theta^{2}\left(\vec{y}, \vec{y}_{1}\right)$.

Then we have

$$
\Psi \vdash_{L^{I}}(\forall \vec{u})(\exists \vec{v}) I_{i}(\vec{u}, \vec{v}), I_{i_{1}}\left(\vec{x}_{1}, \vec{y}_{1}\right),(\exists \vec{u}) \theta^{1}\left(\vec{u}, \vec{x}_{1}\right) \rightarrow(\exists \vec{u}) \theta^{2}\left(\vec{u}, \vec{y}_{1}\right)
$$

Since

$$
(\forall \vec{u})(\exists \vec{v}) I_{i}(\vec{u}, \vec{v}) \in \Psi, \quad \text { we have }
$$

$$
\Psi \underset{L I}{\vdash} I_{i}\left(\vec{x}_{1}, \vec{y}_{1}\right)_{\wedge}\left((\exists \vec{u}) \theta\left(\vec{u}, \vec{x}_{1}\right)\right)^{1} . \supset\left(\left(\exists(\vec{u}) \theta\left(\vec{u}, \vec{y}_{1}\right)\right)^{2} .\right.
$$

Therefore $\quad \Psi \vdash_{L I}\left(\forall \vec{u}_{1}\right)\left(\forall \vec{v}_{1}\right)\left(I_{i_{1}}\left(\vec{u}_{1}, \vec{v}_{1}\right)_{\wedge}\left((\exists \vec{u}) \theta\left(\vec{u}, \vec{u}_{1}\right)\right)^{1} \cdot \supset\left((\exists \vec{u}) \theta\left(\vec{u}, \vec{v}_{1}\right)\right)^{2}\right)$.

This means

$$
(\exists \vec{u}) \theta\left(\vec{u}, w_{1}^{i_{1}}\right) \in \operatorname{In}_{I}(\Psi) .
$$

Q.E.D.

\subsection{Lemma.}

Let $i_{0}<\omega$ and $\vec{w}^{i_{0}}$ be a finite sequence of distinct free variables of length $n$.

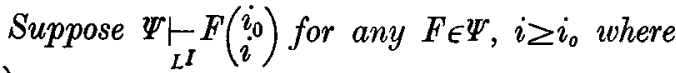
$F\left(\begin{array}{l}i_{0} \\ i\end{array}\right)$ is the sentence obtained from $F$ by replacing every $I_{i_{0}}$ in $F$ by $I_{i}$.

(i) If $(\forall \vec{u})(\exists \vec{v}) \vee I_{i}(\vec{u}, \vec{v}) \in \Psi$, where the length of $\vec{u}, \vec{v}$ is $n, \theta\left(\vec{w}^{i_{0}}\right) \in \operatorname{In}_{I}(\Psi)$ and every free variable in ${ }^{i_{0} \leq i}$ except those in $\vec{w}^{i_{0}}$ belongs to $\bigcup_{i<i_{0}} V_{i}$ then $(\exists \vec{u}) \theta(\vec{u}) \epsilon \operatorname{In}_{I}(\Psi)$.

(ii) If $(\forall \vec{v})(\exists \vec{u})_{i_{0} \leq i} I_{i}(\vec{u}, \vec{v}) \in \Psi$, where the length of $\vec{u}, \vec{v}$ is $n, \theta\left(\vec{w}^{i_{0}}\right) \in \operatorname{In}_{I}(\Psi)$ and every free variable in $\theta$ except those in $\vec{w}^{i_{0}}$ belongs to $\bigcup_{i<i_{0}} V_{i}$ then $(\forall \vec{u}) \theta(\vec{u}) \in \operatorname{In}_{I}(\Psi)$.

Proof We shall prove (i) only.

Assume the hypotheses of (i).

Let $w_{1}^{j}$ be a repetition-free enumeration of free variables in $\theta$ except those in $\vec{w}^{i_{0}}$. Then by the hypotheses of (i),

$$
j_{1}, j_{2}, \cdots, j_{m}<i_{0} \quad \text { where } \quad w_{1}^{j}=\left\langle w_{11}{ }^{j_{1}}, \cdots, w_{1 m}{ }^{{ }^{j}}{ }\right\rangle .
$$

Let $\vec{x}, \vec{y}, \vec{x}_{1}, \vec{y}_{1}$ be finite sequences of free variables such that $\vec{x}, \vec{y}$ have the same length $n, \vec{x}_{1}, \vec{y}_{1}$ have the same length $m$ and all the free variables appearing one of $\vec{x}, \vec{y}, \vec{x}_{1}, \vec{y}_{1}$ are distinct.

Since

$$
\begin{gathered}
\theta\left(\vec{w}^{i_{0}}, w_{1}{ }^{j}\right) \in I n_{I} \Psi . \\
-137-
\end{gathered}
$$




$$
\Psi \vdash_{L^{I}} I_{\boldsymbol{i}_{0}}(\vec{x}, \vec{y}), I_{j}\left(\vec{x}_{1}, \vec{y}_{1}\right), \theta^{1}\left(\vec{x}, \vec{x}_{1}\right) \rightarrow \theta^{2}\left(\vec{y}, \vec{y}_{1}\right) .
$$

Let $i \geq i_{0}$ be an arbitrary integer.

Then $\quad \Psi\left(i_{i}^{i_{0}}\right)_{L^{I}} I_{i}(\vec{x}, \vec{y}), I_{j}^{*}\left(\vec{x}_{1}, \vec{y}_{1}\right), \theta^{1}\left(\vec{x}, \vec{x}_{1}\right) \rightarrow \theta^{2}\left(\vec{y}, \vec{y}_{1}\right)$ because $\quad j_{1}, \cdots, j_{m}<i_{0}$

where $\quad \Psi\left(\begin{array}{l}i_{0} \\ i\end{array}\right)=\left\{F\left(\begin{array}{c}i_{0} \\ i\end{array}\right) ; F \in \Psi\right\}$.

But $\quad \Psi \vdash_{L I} F\left(\begin{array}{l}i_{0} \\ i\end{array}\right) \quad$ for any $F \in \Psi$.

Hence we have

$$
\begin{array}{r}
\Psi \vdash_{L I} I_{i}(\vec{x}, \vec{y}), I_{j}\left(\vec{x}_{1}, \vec{y}_{1}\right), \theta^{1}\left(\vec{x}, \vec{x}_{1}\right) \rightarrow \theta^{2}\left(\vec{y}, \vec{y}_{1}\right) \\
\text { for any } i \geq i_{0} . \\
\text { So, } \quad \Psi \vdash_{L}{\underset{i}{i_{0} \leq i}}_{\vee_{i}} I_{i}(\vec{x}, \vec{y}), I_{j}\left(\vec{x}_{1}, \vec{y}_{1}\right), \theta^{1}\left(\vec{x}, \vec{x}_{1}\right) \rightarrow \theta^{2}\left(\vec{y}, \vec{y}_{1}\right) .
\end{array}
$$

Hence we have

$$
\Psi \vdash_{L^{I}}(\forall \vec{u})(\exists \vec{v}) \underset{i_{0} \leq i}{\wedge} I_{i}(\vec{u}, \vec{v}), I_{j}\left(\vec{x}_{1}, \vec{y}_{1}\right),\left((\exists \vec{u}) \theta\left(\vec{u}, \vec{x}_{1}\right)\right)^{1} \rightarrow\left((\exists \vec{u}) \theta\left(\vec{u}, \vec{y}_{1}\right)\right)^{2}
$$

Since

$$
(\forall \vec{u})(\exists \vec{v}) \underset{i_{0} \leq i}{\vee} I_{i}(\vec{u}, \vec{v}) \in \Psi, \quad \text { we have }
$$

$$
\Psi \vdash_{L I}\left(\forall \vec{u}_{1}\right)\left(\forall \vec{v}_{1}\right)\left(I_{j}\left(\vec{u}_{1}, \vec{v}_{1}\right)_{\wedge}\left((\exists \vec{u}) \theta\left(\vec{u}, \vec{u}_{1}\right)\right)^{1} \supset\left((\exists \vec{u}) \theta\left(\vec{u}, \vec{v}_{1}\right)\right)^{2}\right) .
$$

This means

$$
(\exists \vec{u}) \theta\left(\vec{u}, w_{1}{ }^{\jmath}\right) \in \operatorname{In}_{I}(\Psi) \text {. }
$$

Q.E.D.

\subsection{Lemma.}

Let $\omega_{1}^{i_{1}}, \omega_{2}^{i_{2}}$ be two sequences of distinct free variables such that $\omega_{1}^{i_{1}}, \omega_{2}^{i_{2}}$ are disjoint and $\phi=\phi\left(\omega_{1}{ }^{i_{1}}, \omega_{2}{ }^{i_{2}}\right)$ be a formula in $\mathscr{L}$ whose free variables are exactly those in $\omega_{1}{ }^{i_{1}}, \omega_{2}^{i_{2}}$.

Suppose $\left(\forall \vec{u}_{2}\right)\left(\forall \vec{u}_{1}\right)\left(\forall \vec{v}_{2}\right)\left(\forall \vec{v}_{1}\right)\left(I_{i_{1}}\left(\vec{u}_{1}, \vec{v}_{1}\right)_{\wedge} I_{i_{2}}\left(\vec{u}_{2}, \vec{v}_{2}\right)_{\wedge} \Phi^{1}\left(\vec{u}_{1}, \vec{u}_{2}\right) . \supset \phi^{2}\left(\vec{v}_{1}, \vec{v}_{2}\right)\right) \in \Psi$.

(i) If $\left(\forall \vec{u}_{2}\right)\left(\forall \vec{v}_{2}\right)\left(\forall \vec{u}_{1}\right)\left(\exists \vec{v}_{1}\right)\left(\varphi^{1}\left(\vec{u}_{1}, \vec{u}_{2}\right)_{\Lambda} I_{i_{2}}\left(\vec{u}_{2}, \vec{v}_{2}\right) . \supset I_{i_{1}}\left(\vec{u}_{1}, \vec{v}_{1}\right)\right) \in \Psi$

$$
\text { and } \theta\left(w_{1}^{i_{1}}\right) \in \operatorname{In}_{I}(\Psi) \text {, then }(\exists \vec{u})\left(\varphi\left(\vec{u}, w_{2}{ }^{i_{2}}\right)_{\wedge} \theta(\vec{u})\right) \in \operatorname{In}_{I}(\Psi) \text {. }
$$

(ii) If $\left(\forall \vec{v}_{2}\right)\left(\forall \vec{u}_{2}\right)\left(\forall \vec{v}_{1}\right)\left(\exists \vec{u}_{1}\right)\left(\varphi^{2}\left(\vec{v}_{1}, \vec{v}_{2}\right)_{\wedge} I_{i_{2}}\left(\vec{u}_{2}, \vec{v}_{2}\right) \supset I_{i_{1}}\left(\vec{u}_{1}, \vec{v}_{1}\right)\right) \in \Psi$

$$
\text { and } \theta\left(w_{1}^{{ }_{1}{ }_{1}}\right) \in \operatorname{In}_{I}(\Psi) \text {, then }(\forall \vec{u})\left(\phi\left(\vec{u}, w_{2}{ }^{{ }_{2}}{ }^{2}\right) \supset \theta(\vec{u})\right) \in I n_{I}(\Psi) \text {. }
$$

Proof we shall prove (i) only.

Assume $\quad\left(\forall \vec{u}_{2}\right)\left(\forall \vec{v}_{2}\right)\left(\forall \vec{u}_{1}\right)\left(\exists \vec{v}_{1}\right)\left(\Phi^{1}\left(\vec{u}_{1}, \vec{u}_{2}\right)_{\lambda} I_{i_{2}}\left(\vec{u}_{2}, \vec{v}_{2}\right) . \supset I_{i_{1}}\left(\vec{u}_{1}, \vec{v}_{1}\right)\right) \in \Psi \quad$ and $\theta\left(\omega_{1}{ }^{i_{1}}\right) \in \operatorname{In}_{I}(\Psi)$.

Let $w_{\mathbf{3}}{ }^{i}$, be a repetition-free enumeration of free variables in $\theta$ except those in $w_{1}^{i_{1}}$ or $w_{2}^{i_{2}}$.

Let $\vec{x}_{1}, \vec{x}_{2}, \vec{x}_{3}, \vec{y}_{1}, \vec{y}_{2}, \vec{y}_{3}$ be such that $\vec{x}_{1}, \vec{y}_{1}, w_{1}{ }^{i_{1}}$ have the same length $n_{1}, \vec{x}_{2}, \vec{y}_{2}, w_{2}{ }^{i_{3}}$ 
have the same length $n_{2}, \vec{x}_{3}, \vec{y}_{3}, w_{3}{ }^{i_{3}}$ have the same length $n_{3}$ and all the free variables appearing one of these sequences are distinct.

Since $\quad \theta\left(w_{1}^{i_{1}}, w_{2}^{i_{3}}, w_{3}{ }^{i_{3}}\right) \in \operatorname{In}_{1}(\Psi)$

$$
\Psi \vdash_{L I} I_{i_{1}}\left(\vec{x}_{1}, \vec{y}_{1}\right), I_{i_{8}}\left(\vec{x}_{2}, \vec{y}_{2}\right), I_{i_{8}}\left(\vec{x}_{3}, \vec{y}_{3}\right), \theta^{1}\left(\vec{x}_{1}, \vec{x}_{2}, \vec{x}_{3}\right) \rightarrow \theta^{2}\left(\vec{y}_{1}, \vec{y}_{2}, \vec{y}_{3}\right) \text {. }
$$

By the hypotheses of this lemma

$$
\Psi \underset{{ }^{I} I}{\vdash} I_{i_{1}}\left(\vec{x}_{1}, \vec{y}_{1}\right), I_{i_{2}}\left(\vec{x}_{2}, \vec{y}_{2}\right), \phi^{1}\left(\vec{x}_{1}, \vec{x}_{2}\right) \rightarrow \phi^{2}\left(\vec{y}_{1}, \vec{y}_{2}\right) .
$$

By $\left(^{*}\right)$ and $(* *)$ we have

$$
\begin{aligned}
\Psi \vdash_{L I} I_{i_{8}}\left(\vec{x}_{2}, \vec{y}_{2}\right), I_{i_{3}}\left(\vec{x}_{3}, \vec{y}_{3}\right), \phi^{1}\left(\vec{x}_{1}, \vec{x}_{2}\right)_{\Lambda} I_{i_{2}}\left(\vec{x}_{2}, \vec{y}_{2}\right) . \supset I_{i_{1}}\left(\vec{x}_{1}, \vec{y}_{1}\right), \\
\varphi^{1}\left(\vec{x}_{1}, \vec{x}_{2}\right)_{\Lambda} \theta^{1}\left(\vec{x}_{1}, \vec{x}_{2}, \vec{x}_{3}\right) \rightarrow \phi^{2}\left(\vec{y}_{1}, \vec{y}_{2}\right)_{\wedge} \theta^{2}\left(\vec{y}_{1}, \vec{y}_{2}, \vec{y}_{3}\right) .
\end{aligned}
$$

Hence $\Psi \vdash_{L I}\left(\forall \vec{u}_{2}\right)\left(\forall \vec{v}_{2}\right)\left(\forall \vec{u}_{1}\right)\left(\exists \vec{v}_{1}\right)\left(\phi^{\mathbf{1}}\left(\vec{u}_{1}, \vec{u}_{2}\right)_{\Lambda} I_{i_{2}}\left(\vec{u}_{2}, \vec{v}_{2}\right) . \supset I_{i_{1}}\left(\vec{u}_{1}, \vec{v}_{1}\right)\right)$,

$$
\begin{aligned}
& I_{i_{2}}\left(\vec{x}_{2}, \vec{y}_{2}\right), I_{i_{8}}\left(\vec{x}_{3}, \vec{y}_{3}\right),(\exists \vec{u})\left(\varphi^{1}\left(\vec{u}, \vec{x}_{2}\right)_{\wedge} \theta^{1}\left(\vec{u}, \vec{x}_{2}, \vec{x}_{3}\right)\right) \\
& \rightarrow(\exists \vec{u})\left(\phi^{2}\left(\vec{u}, \vec{y}_{2}\right)_{\Lambda} \theta^{2}\left(\vec{u}, \vec{y}_{2}, \vec{y}_{3}\right)\right) .
\end{aligned}
$$

Since $\quad\left(\forall \vec{u}_{2}\right)\left(\forall \vec{v}_{2}\right)\left(\forall \vec{u}_{1}\right)\left(\exists \vec{v}_{1}\right)\left(\left(\Phi^{1}\left(\vec{u}_{1}, \vec{u}_{2}\right)_{\Lambda} I_{i_{3}}\left(\vec{u}_{2}, \vec{v}_{2}\right) . \supset I_{i_{1}}\left(\vec{u}_{1}, \vec{v}_{1}\right)\right) \in \Psi\right.$, this means $\quad(\exists \vec{u})\left(\varphi\left(\vec{u}, w_{2}{ }^{{ }^{i_{2}}}\right)_{\wedge} \theta\left(\vec{u}, w_{2}{ }^{i_{2}}, w_{3}{ }^{{ }^{i}}\right)\right) \in \operatorname{In}_{I}(\Psi)$.

Q.E.D.

\subsection{Lemma.}

Let $\psi\left(w^{i}\right)$ be a formula in $\mathscr{L}$, where $w^{2}$ is repetition-free enumeration of free variables in $\psi$.

(i) If $(\forall \vec{u})(\exists \vec{v})\left(\psi^{1}(\vec{u}) \supset . I_{i}(\vec{u}, \vec{v}){ }_{\wedge} \psi^{2}(\vec{v})\right) \in \Psi \quad$ and $\quad \theta\left(w^{i}\right) \in I_{I}(\Psi)$,

then $(\exists \vec{u})\left(\psi(\vec{u})_{\wedge} \theta(\vec{u})\right) \in \operatorname{In}_{I}(\Psi)$.

(ii) If $(\forall \vec{v})(\exists \vec{u})\left(\psi^{2}(\vec{v}) . \supset I_{i}(\vec{u}, \vec{v})_{\wedge} \psi^{1}(\vec{u})\right) \in \Psi \quad$ and $\quad \theta\left(w^{2}\right) \in \operatorname{In}_{I}(\Psi)$,

$$
\text { then }(\forall \vec{u})(\Psi(\vec{u}) \supset \theta(\vec{u})) \in \operatorname{In}_{I}(\Psi) \text {. }
$$

Proof we shall prove (i) only.

Assume $(\forall \vec{u})(\exists \vec{v})\left(\psi^{1}(\vec{u}) \supset . I_{\boldsymbol{i}}(\vec{u}, \vec{v})_{\wedge} \psi^{2}(\vec{v})\right) \epsilon \Psi$ and $\theta\left(w^{\vec{i}}\right) \epsilon \operatorname{In}_{I}(\Psi)$. in $w^{i}$

Let $w_{1}^{j}$ be a repetition-free enumeration of free variables in $\theta\left(w^{i}\right)$ except those

Let, $\vec{x}, \vec{x}_{1}, \vec{y}, \vec{y}_{1}$, be such that $\vec{x}, \vec{y}_{1}, w^{i}$ have the same length, $\vec{x}_{1}, \vec{y}_{1}, w_{1}{ }^{\vec{j}}$ have the same length and all the free variables appearing one of these sequences are distinct.

Since $\quad \theta\left(w^{i}, w_{1}^{j}\right) \in \operatorname{In}_{I}(\Psi), \quad$ we have

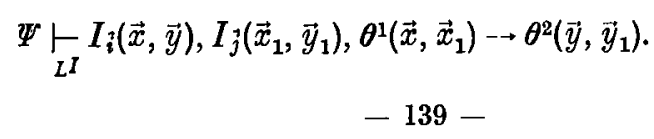


Hence $\quad \Psi \underset{L^{I}}{\vdash} \psi^{1}(\vec{x}) \supset I_{i}(\vec{x}, \vec{y})_{\wedge} \psi^{2}(\vec{y}), I_{j}\left(\vec{x}_{1}, \vec{y}_{1}\right), \psi^{1}(\vec{x})_{\wedge} \theta^{1}\left(\vec{x}, \vec{x}_{1}\right) \rightarrow \psi^{2}(\vec{y})_{\wedge} \theta^{2}\left(\vec{y}, \vec{y}_{1}\right)$

Therefore $\quad \Psi \underset{L^{I}}{\vdash}(\forall \vec{u})(\exists \vec{v})\left(\psi^{1}(\vec{u}) \supset . I_{i}(\vec{u}, \vec{v})_{\wedge} \psi^{2}(\vec{v})\right), I_{j}\left(\vec{x}_{1}, \vec{y}_{1}\right)$,

$$
(\exists \vec{u})\left(\psi^{1}(\vec{u})_{\Lambda} \theta^{1}\left(\vec{u}, \vec{x}_{1}\right)\right) \rightarrow(\exists \vec{u})\left(\psi^{2}(\vec{u})_{\Lambda} \theta^{2}\left(\vec{u}, \vec{y}_{1}\right)\right) .
$$

Since $\quad(\forall \vec{u})(\exists \vec{v})\left(\psi^{1}(\vec{u}) \supset . I_{i}(\vec{u}, \vec{v})_{\wedge} \psi^{2}(\vec{v})\right) \in \Psi, \quad$ this means

$$
(\exists \vec{u})\left(\psi(\vec{u})_{\wedge} \theta\left(\vec{u}, w_{1}{ }^{j}\right) \in \operatorname{In}_{I}(\Psi) .\right.
$$

The above lemmas 1.7-1.11 show us some important properties of $\operatorname{In}_{I}(\Psi)$, which are related to the definitions of $I$-primitive sentences, $I$-primitive sets and $\Delta_{I}(\Psi)$ in the next section.

\section{$\S 2$ I-primitive sets.}

Let $w^{i}, w_{1}^{j}$ be two finite sequences of distinct free variables such that $w^{i}$, $w_{\mathbf{1}}{ }^{\vec{j}}$ are disjoint.

Suppose $\psi\left(w^{i}\right), \varphi\left(w^{i}, w_{1}^{j}\right)$ are formulas in $\mathscr{L}$ such that all the free variables in $\psi$ are exactly those in $w^{i}$ and all the free variables in $\boldsymbol{\varphi}$ are exactly those in $w^{i}$ or $w_{1}^{j}$.

\subsection{Definition.}

(1) $(\forall \vec{u})(\forall \vec{v})\left(I_{i}(\vec{u}, \vec{v})_{\wedge} \psi^{1}(\vec{u}) . \supset \psi^{2}(\vec{v})\right)$ is the $I$-primitive sentence of type 1 defined by $\psi\left(w^{i}\right)$.

(2) $(\forall \vec{u})(\exists \vec{v}) I_{i}(\vec{u}, \vec{v})$ is the $I$-primitive sentence of type 2 denfied by $\vec{i}$.

(3) $(\forall \vec{v})(\exists \vec{u}) I_{i}(\vec{u}, \vec{v})$ is the $I$-primitive sentence of type 3 defined by $\vec{i}$.

(4) $(\forall \vec{u})(\exists \vec{v}) \vee I_{i_{0} \leq i} I_{i}(\vec{u}, \vec{v})$ is the $I$-primitive sentence of type 4 defined by $\left(i_{0}, n\right)$, where $n$ is the length of $\vec{u}$.

(5) $(\forall \vec{v})(\exists \vec{u}) \sum_{i_{0} \leq i} I_{i}(\vec{u}, \vec{v})$ is the $I$-primitive sentence of type 5 defined by $\left(i_{0}, n\right)$, where $n$ is the length of $\vec{v}$.

(6) $\left(\forall \vec{u}_{2}\right)\left(\forall \vec{v}_{2}\right)\left(\forall \vec{u}_{1}\right)\left(\exists \vec{v}_{1}\right)\left(\varphi^{1}\left(\vec{u}_{1}, \vec{u}_{2}\right)_{\Lambda} I_{j}\left(\vec{u}_{2}, \vec{v}_{2}\right) . \supset I_{i}\left(\vec{u}_{1}, \vec{v}_{1}\right)\right)$ is the $I$-primitive sentence of type 6 defined by $\left(\boldsymbol{\varphi}, w^{i}\right)$.

(7) $\left(\forall \vec{v}_{2}\right)\left(\forall \vec{u}_{2}\right)\left(\forall \vec{v}_{1}\right)\left(\exists \vec{u}_{1}\right)\left(\varphi^{2}\left(\vec{v}_{1}, \vec{v}_{2}\right)_{\wedge} I_{j}\left(\vec{u}_{2}, \vec{v}_{2}\right) \cdot \supset I_{i}\left(\vec{u}_{1}, \vec{v}_{1}\right)\right)$ is the $I$-primitive sentence of type 7 defined by $\left(\varphi, w^{i}\right)$.

(8) $(\forall \vec{u})(\exists \vec{v})\left(\psi^{\mathrm{l}}(\vec{u}) \supset . I_{i}(\vec{u}, \vec{v})_{\wedge} \psi^{2}(\vec{v})\right)$ is the $I$-primitive sentence of type 8 defined by $\psi\left(w^{i}\right)$.

(9) $(\forall \vec{v})(\exists \vec{u})\left(\psi^{2}(\vec{v}) \supset . I_{i}(\vec{u}, \vec{v}) \wedge \psi^{1}(\vec{u})\right)$ is the $I$-primitive sentence of type 9 defined by $\psi\left(w^{i}\right)$. 
A sentence $F$ in $L^{I}$ is $I$-primitive if $F$ satisfies one of the conditions (1)-(9).

Notice that the above definitions correspond to lemma $1.7 \sim$ lemma 1.11 .

\subsection{Definition.}

A set $\Psi$ of sentences in $L^{I}$ is $I$-primitive if

(1) every sentence in $\Psi$ is $I$-primitive,

(2) if $\Psi$ has the $I$-primitive sentence of type 6 (or 7$)$ defined by $\left(\varphi, w^{\vec{i}}\right)$, then $\Psi$ has the $I$-primitive sentence of type 1 defined by $\varphi$,

(3) if $\Psi$ has the $I$-primitive sentence of type 4 (or 5) defined by $\left(i_{0}, n\right)$, then

(a) $\Psi$ has the $I$-primitive sentence of type 4 (or 5 ) defined by $\left(i_{0}+1, n\right.$ ),

(b) if $F \in \Psi$ is an $I$-primitive sentence whose type is neither 4 nor 5 and $i \geq i_{0}$ then $F\left(\begin{array}{l}i_{o} \\ i\end{array}\right) \in \Psi$.

The condition (2) above corresponds to lemma 1.10 and the conditions (3) above correspond to lemma 1.9 .

\subsection{LemMa.}

Suppose $\Psi$ is an I-primitive set.

If $\Psi$ has an I-primitive sentence of type 4 or 5 defined by $\left(i_{a}, n\right)$, then $\Psi \vdash_{L I} F\left(\begin{array}{l}i_{0} \\ i\end{array}\right)$ for any $F \in \Psi, i \geq i_{0}$.

Proof Assume that $\Psi$ has an $I$-primitive sentence of type 4 or 5 defined by $\left(i_{0}, n\right)$.

If $F \in \Psi$ is not of type 4 or 5 , then by 2.2 , (3), (b) $F\left(\begin{array}{l}i_{0} \\ i\end{array}\right) \in \Psi$ for any $i \geq i_{0}$. Hence we have $\Psi \vdash_{L} F\left(i_{i}\right)$ for any $F \in \Psi$ whose type is neither 4 nor 5 .

So, we suffice to prove only the case that $F$ is of type 4 or 5 . But we can assume, without loss of generality that $F$ is of type 4 .

Suppose $F$ is the $I$-primitive sentence of type 4 defined by $\left(j_{0}, l\right)$. If $j_{0}>i_{0}$ then $F\left(\begin{array}{l}i_{0} \\ i\end{array}\right)=F$ for any $i \geq i_{0}$, hence $\Psi \underset{L^{I}}{\vdash} F\left(\begin{array}{c}i_{0} \\ i\end{array}\right)$ for any $i \geq i_{0}$. So, assume $j_{0} \leq i_{0}$. Let $i \geq i_{0}$.

Then by 2.2 (3), @, $\Psi$ has the primitive sentence $F_{1}$ of type 4 defined by $(i, l)$.

Since

$$
\begin{aligned}
& \vdash_{L I} F_{1} \supset F\left(\begin{array}{l}
i_{0} \\
i
\end{array}\right), \text { we have } \\
& \Psi \vdash_{L I} F\left(\begin{array}{l}
i_{0} \\
i
\end{array}\right) .
\end{aligned}
$$

\subsection{Definition.}

For each $I$-primitive set $\Psi$, we define $\Delta_{I}(\Psi)$ by the following rules:

(1) If $\Psi$ has the $I$-primitive sentence of type 1 defined by $\psi$, then $\psi \in \Delta_{I}(\Psi)$.

(2) If $\Psi$ has the $I$-primitive sentence of type 2 defined by $\vec{i}$ and $\theta\left(w^{i}\right) \in \Delta_{I}(\Psi)$, then $(\exists \vec{u}) \theta(\vec{u}) \in \Delta_{I}(\Psi)$.

(3) If $\Psi$ has the $I$-primitive sentence of type 3 defined by $\vec{i}$ and $\theta\left(w^{i}\right) \in \Delta_{I}(\Psi)$, then 
$(\forall \vec{u}) \theta(\vec{u}) \in \Delta_{I}(\Psi)$.

(4) If $\Psi$ has the $I$-primitive sentence of type 4 defined by $\left(i_{0}, n\right)$ and $\theta\left(\vec{w}^{i}\right) \in \Delta_{I}(\Psi)$ where the length of $\vec{w}^{i_{0}}$ is $n$ and every free variable in $\theta$ except those in $\vec{w}^{i_{0}}$ belongs to $\bigcup_{i<i_{0}} V_{i}$, then $(\exists \vec{u}) \theta(\vec{u}) \in \Delta_{I}(\Psi)$.

(5) If $\Psi$ has the $I$-primitive sentence of type 5 defined by $\left(i_{0}, n\right)$ and $\theta\left(\vec{w}^{i_{0}}\right) \epsilon \Delta_{I}(\Psi)$, where the length of $\vec{w}^{i_{0}}$ is $n$ and every free variable in $\theta$ except those in $\vec{w}^{i_{0}}$ belongs to $\bigcup_{i<i_{0}} V_{i}$, then $(\forall \vec{u}) \theta(\vec{u}) \in \Delta_{I}(\Psi)$.

(6) If $\Psi$ has the $I$-primitive sentence of type 6 defined by $\left(\varphi\left(w^{i}\right), w^{i}\right)$ and $\theta\left(w^{i}\right) \in \Delta_{I}(\Psi)$, then $(\exists \vec{u})\left(\varphi(\vec{u})_{\Lambda} \theta(\vec{u})\right) \in \Delta_{I}(\Psi)$.

(7) If $\Psi$ has the $I$-primitive sentence of type 7 defined by $\left(\boldsymbol{\varphi}\left(w^{i}\right), w^{\frac{i}{2}}\right)$ and $\theta\left(w^{\frac{i}{2}}\right) \in \Delta_{I}(\Psi)$, then $(\forall \vec{u})(\varphi(\vec{u}) \supset \theta(\vec{u})) \in \Delta_{I}(\Psi)$.

(8) If $\Psi$ has the $I$-primitive sentence of type 8 defined by $\psi\left(w^{i}\right)$ and $\theta\left(v v^{i}\right) \in \Delta_{I}(\Psi)$, then $(\exists \vec{u})(\psi(\vec{u}) \wedge \theta(\vec{u})) \in \Delta_{I}(\Psi)$.

(9) If $\Psi$ has the $I$-primitive sentence of type 9 defined by $\psi\left(w^{i}\right)$ and $\theta\left(w^{i}\right) \in \Delta_{I}(\Psi)$, then $(\forall \vec{u})(\psi(\vec{u}) \supset \theta(\vec{u})) \in \Delta_{I}(\Psi)$.

(11) $T, \perp \in \Delta_{l}(\Psi)$.

(11) If $\emptyset$ is a non empty, countable set of formulas in $\Delta_{I}(\Psi)$ such that only finitely many free variables occur in $\Phi$ then $\wedge \emptyset, \vee \emptyset \in \Delta_{I}(\Psi)$.

(12) If $\theta\left(w^{i}\right) \in \Delta_{I}(\Psi)$, then $\theta\left(w_{1}^{i}\right) \in \Delta_{I}(\Psi)$, where $w^{i}$ is a finite sequence of distinct free variables and $w^{i}=\left\langle w_{1}^{i_{1}}, \cdots, w_{n}{ }^{i n}\right\rangle, w_{1}{ }^{i}=\left\langle w_{11}{ }^{i_{1}}, \cdots, w_{1 n}{ }^{i_{n}}\right\rangle$.

(13) All the formulas in $\Delta_{I}(\Psi)$ are obtained from (1)-(12).

Then by lemmas $1.7 \sim 1.11$ and lemma 2.3 , we have the following lemma 2.5 .

\subsection{Lemma.}

For any I-primitive set $\Psi$

$$
\Delta_{I}(\Psi) \subseteq \operatorname{In}_{I}(\Psi)
$$

\subsection{Definition.}

For each $I$-primitive sentence $F$, we define $(F)$-rules by the followings;

(1) If $F$ is the $I$-primitive sentence of type 1 defined by $\boldsymbol{\phi}\left(w^{\imath}\right)$, then

$$
\text { (F) } \frac{\Gamma \rightarrow \Theta, I_{i_{1}}\left(x_{1}, y_{1}\right) \cdots \cdots \cdots \Gamma \rightarrow \Theta, I_{i_{n}}\left(x_{n}, y_{n}\right)}{F, \Gamma \rightarrow \Theta},
$$

where $\psi^{1}(\vec{x}) \in \Gamma$ and $\psi^{2}(\vec{y}) \in \Theta$.

(2) If $F$ is the $I$-primitive sentence of type 2 defined by $\vec{i}$ then

$$
(F) \frac{I_{i_{1}}\left(x_{1}, y_{1}\right), \cdots, I_{i_{n}}\left(x_{n}, y_{n}\right), \Gamma \rightarrow \Theta}{F, \Gamma \rightarrow \Theta}
$$

where $y_{1}, \cdots, y_{n}$ are all distinct, different from $x_{1}, \cdots, x_{n}$ and don't appear in $\Gamma, \Theta$. 
(3) If $F$ is the $I$-primitive sentence of type 3 defined by $\vec{i}$ then

$$
\text { (F) } \frac{I_{i_{1}}\left(x_{1}, y_{1}\right), \cdots, I_{i_{n}}\left(x_{n}, y_{n}\right), \Gamma \rightarrow \Theta}{F, \Gamma \rightarrow \Theta}
$$

where $x_{1}, \cdots, x_{n}$ are all distinct, different from $y_{1}, \cdots, y_{n}$ and don't appear in $r, \Theta$.

(4) If $F$ is the $I$-primitive sentence of type 4 defined by $\left(i_{0}, n\right)$, then

$$
\text { (F) } \frac{I_{i}\left(x_{1}, y_{1}\right), \cdots, I_{i}\left(x_{n}, y_{n}\right), \Gamma \rightarrow \Theta\left(i \geq i_{0}\right)}{F, \Gamma \rightarrow \Theta},
$$

where $y_{1}, \cdots, y_{n}$ are all distinct, different from $x_{1} \cdots, x_{n}$ and don't appear in, $\Gamma, \Theta$.

(5) If $F$ is the $I$-primitive sentence of type 5 defined by $\left(i_{0}, n\right)$, then

$$
\text { (F) } \frac{I_{i}\left(x_{1}, y_{1}\right), \cdots, I_{i}\left(x_{n}, y_{n}\right), \Gamma \rightarrow \Theta\left(i \geq i_{0}\right)}{F, \Gamma \rightarrow \Theta}
$$

where $x_{1} \cdots, x_{n}$ are all distinct, different from $y_{1}, \cdots, y_{n}$ and don't appear in $\Gamma, \Theta$.

(6) If $\boldsymbol{F}$ is the $I$-primitive sentence of type 6 defined by $\left(\boldsymbol{\phi} w^{i}\right)$, where $\boldsymbol{\phi}=\boldsymbol{\phi}\left(w^{i}\right.$, $\left.w_{1}{ }^{j}\right)$, then

$$
(F) \frac{\Gamma \rightarrow \Theta, I_{j_{1}}\left(x_{11}, y_{11}\right) \cdots \Gamma \rightarrow \Theta, I_{j_{m}}\left(x_{1_{m}}, y_{1_{m}}\right) \quad I_{i_{1}}\left(x_{1}, y_{1}\right), \cdots, I_{i_{n}}\left(x_{n}, y_{n}\right), \Gamma \rightarrow \Theta}{F, \Gamma \rightarrow \Theta} \text {, }
$$

where $\varphi^{\mathrm{i}}\left(\vec{x}, \vec{x}_{1}\right) \epsilon \Gamma, y_{1}, \cdots, y_{n}$ are all distinct, different from $x_{1}, \cdots, x_{n}, y_{11}, \cdots$, $y_{1 m}, x_{11}, \cdots, x_{1 m}$ and don't appear in $\Gamma, \Theta$.

(7) If $F$ is the $I$-primitive sentence of type 7 defined by $\left(\boldsymbol{\phi}, w^{i}\right)$, where $\boldsymbol{\phi}=\boldsymbol{\phi}\left(w^{i}\right.$, $\left.w_{1}{ }^{3}\right)$, then

$$
\text { (F) } \frac{\Gamma \rightarrow \Theta, I_{j_{1}}\left(x_{11}, y_{11}\right) \cdots \Gamma \rightarrow \Theta, I_{j_{m}}\left(x_{1 m}, y_{1 m}\right) \quad I_{i_{1}}\left(x_{1}, y_{1}\right), \cdots, I_{i_{n}}\left(x_{n}, y_{n}\right), \Gamma \rightarrow \Theta}{F, \Gamma \rightarrow \Theta} \text {, }
$$

where $\varphi^{2}\left(\vec{y}, \vec{y}_{1}\right) \epsilon \Gamma, x_{1}, \cdots, x_{n}$ are all distinct, different from $y_{1}, \cdots, y_{n}, x_{11}, \cdots$, $x_{1 m}, y_{11}, \cdots, y_{1 m}$ and don't appear in $\Gamma, \Theta$.

(8) If $F$ is the $I$-primitive sentence of type 8 defined by $\psi\left(w^{2}\right)$, then

$$
\text { (F) } \frac{I_{i_{1}}\left(x_{1}, y_{1}\right), \cdots, I_{i_{n}}\left(x_{n}, y_{n}\right), \psi^{2}(\vec{y}), \Gamma \rightarrow \Theta}{F, \Gamma \rightarrow \Theta},
$$

where $\psi^{1}\left(x_{1}, \cdots, x_{n}\right) \in \Gamma, y_{1}, \cdots, y_{n}$ are all distinct, different from $x_{1}, \cdots, x_{n}$ and don't appear in $\Gamma, \Theta$.

(9) If $F$ is the $I$-primitive sentence of type 9 defined by $\psi\left(w^{i}\right)$ then

$$
\begin{gathered}
(F) \frac{I_{i_{1}}\left(x_{1}, y_{1}\right), \cdots, I_{i_{n}}\left(x_{n}, y_{n}\right), \psi^{1}(\vec{x}), \Gamma \rightarrow \Theta}{F, \Gamma \rightarrow \Theta}, \\
-143-
\end{gathered}
$$


where $\psi^{2}\left(y_{1}, \cdots, y_{n}\right) \epsilon \Gamma, x_{1}, \cdots, x_{n}$ are all distinct, different from $y_{1}, \cdots, y_{n}$ and don't appear in $\Gamma, \Theta$.

We sometimes call $(F)$-rules $(j)$-rules for each primitive sentence $F$ of type $j, j=1, \cdots, 9$.

We should remark that in the definition 2.6 , we always assume that $\psi\left(w^{i}\right)$, $\varphi\left(w^{\vec{i}}, w_{1}{ }^{j}\right)$ have the same meanings as in 2.1 .

\subsection{Definition.}

Let $\Psi$ be an $I$-primitive set. A sequent $I \rightarrow \Theta$ in $L^{I}$ is said to be a $\Psi$-sequent if $r$ consists of 1-formulas, 2-formulas, $F$ for $F \in \Psi, I_{i}(x, y)$ for $i<\omega, x, y$,

$\Theta$ consists of 1-formulas, 2-formulas, $I_{i}(x, y)$ for $i<\omega, x, y$ and there are only finitely many formulas of the form $I_{i}(x, y)$ for some $i<\omega, x, y$ in $\Gamma$.

Let $\Gamma \rightarrow \Theta$ be a $\Psi$-sequent. Then a $\Psi$-derivation $\mathscr{D}$ of $\Gamma \rightarrow \Theta$ is an arrangement of $\Psi$-sequents in tree form (possibly infinite) such that

(1) the lowest sequent of $\mathscr{D}$ is $\Gamma \rightarrow \Theta$,

(2) all the uppermost sequents in $\mathscr{D}$ are all axiom sequents,

(3) consecutive sequents in any branch of the tree are connected by one of the following rules;

(a) (w)-rules,

(b) cut rules whose cut formulas are 1-formulas or 2-formulas and logical inference rules whose principal formulas are 1-formulas or 2-formulas,

(C) $(F)$-rules for $F \in \Psi$.

Then.

\subsection{LemMa.}

For any $I$-primitive set $\Psi$, any $\Psi$-sequent $\Gamma \rightarrow \Theta$ if $\underset{L I}{\vdash} \Gamma \rightarrow \Theta$ then there is a $\Psi$ derivation of $\Gamma \rightarrow \Theta$.

Proof This is an immediate consequence of normal derivation theorem in [27].

Q.E.D.

\section{$\S 3 I$-interpolation theorem and $I$-characterization theorem on $I$-primitive sets.}

In this section, we shall prove the main theorems of this Chapter, i.e. I-interpolation theorem and $I$-characterization theorem on $I$-primitive sets.

Let $f_{i} ; F V \times F V \rightarrow V_{i}$ be a fixed bijection for each $i<\omega$.

By $\nu, \tau$ (with or without suffixes) we shall denote finite subsets of $\omega \times F V \times$ FV.

For any $I$-primitive set $\Psi$ and any $\Psi$-derivation $\mathscr{D}$ of a $\Psi$-sequent $\Gamma \rightarrow \Theta$ we shall associate a sequence $n(\mathscr{D})=\langle i, x, y\rangle$ or $\langle\tau, \theta\rangle$ such that 
$\left.{ }^{*}\right)$ if $n(\mathscr{D})=\langle i, x, y\rangle$ then $I_{i}(x, y) \in \Gamma_{\mathrm{n}} \Theta$,

$\left(^{* *}\right)$ if $n(\mathscr{D})=\langle\tau, \theta\rangle$ then

(1) $\theta \in \Delta_{I}(\Psi)$,

(2) the set of free variables in $\theta$ is exactly $\left\{f_{i}(x, y):\langle i, x, y>\epsilon \tau\}\right.$,

(3) $I_{i}(x, y) \epsilon \Gamma$ each for $\langle i, x, y,>\epsilon \tau$,

(4) $\vdash_{\mathscr{S}} \Gamma(1) \rightarrow \Theta(1), \theta\left(\begin{array}{l}\cdots f_{i}(x, y) \cdots \\ \cdots \cdots\end{array}\right)$,

(5) $\vdash_{\mathscr{S}} \Gamma(2), \theta\left(\begin{array}{c}\cdots f_{i}(x, y) \cdots \\ \cdots \cdots y \cdots \cdots\end{array}\right) \rightarrow \Theta(2)$.

\subsection{Definition of $n(\mathscr{D})$.}

Case $1 \mathscr{D}$ is an axiom sequent,

$$
\begin{aligned}
& \text { if } \mathscr{D} \text { is } \theta^{1} \rightarrow \theta^{1} \text { or } \rightarrow \theta^{1}, \quad \text { let } n(\mathscr{D})=\langle\phi, \perp\rangle, \\
& \text { if } \mathscr{D} \text { is } \theta^{2} \rightarrow \theta^{2} \text { or } \rightarrow \theta^{2}, \quad \text { let } n(\mathscr{D})=\langle\phi, \top\rangle, \\
& \text { if } \mathscr{D} \text { is } I_{i}(x, y) \rightarrow I_{i}(x, y), \text { let } n(\mathscr{D})=\langle i, x, y\rangle .
\end{aligned}
$$

Let $R$ be the last rule of $\mathscr{D}$.

Case $2 R$ is not one of the following rules; $(C),(\vee \rightarrow),(\rightarrow \wedge),(F)$-rules.

Then $\mathscr{D}$ has the form $\quad R \frac{\mathscr{D}_{1}}{\Gamma \rightarrow \Theta}$

Let $n(\mathscr{D})=n\left(\mathscr{D}_{1}\right)$.

Case $3 \quad R$ is $(C)$, then

$$
\left\lfloor\mathscr{D}_{1} \quad \downarrow \mathscr{P}_{2}\right.
$$

$\mathscr{D}$ has the form

$$
R \frac{\Gamma_{1} \rightarrow \Theta_{1} \quad \Gamma_{2} \rightarrow \Theta_{2}}{\Gamma_{1}, \Gamma_{2}-F \rightarrow \Theta_{1}-F, \Theta_{2}},
$$

if $n\left(\mathscr{D}_{1}\right)=\langle i, x, y\rangle$ or $n\left(\mathscr{D}_{2}\right)=\langle i, x, y\rangle$, let $n(\mathscr{D})=\langle i, x, y\rangle$,

if $n\left(\mathscr{D}_{1}\right)=\left\langle\boldsymbol{\tau}_{1}, \theta_{1}\right\rangle$ and $n\left(\mathscr{D}_{2}\right)=\left\langle\tau_{2}, \theta_{2}\right\rangle$,

let $n(\mathscr{D})=\left\langle\tau^{\cup} \tau_{2}, \theta_{1}{ }^{\vee} \theta_{2}\right\rangle$ provided $F$ is 1-formula,

Let $n(\mathscr{D})=\left\langle\tau_{1}{ }^{\cup} \tau_{2}, \theta_{1 \wedge} \theta_{2}\right\rangle$ provided $F$ is 2-formula.

Case $4 \quad R$ is $(\mathrm{V} \rightarrow)$, then

$$
\mathscr{D} \text { has the form } \quad R \frac{\mathfrak{D}_{F}}{\vee K, \Gamma \rightarrow \Theta(F \in K)},
$$

if $n(\mathscr{D})=\langle i, x, y\rangle$ for some $F \in K$, let $n(\mathscr{D})=\langle i, x, y\rangle$, if $n\left(\mathscr{D}_{F}\right)=\left\langle\tau_{F}, \theta_{F}\right\rangle$ for all $F \in K$, let $n(\mathscr{D})=\left\langle\underset{F \in K}{\cup} \tau_{F}, \underset{F \in K}{\vee} \theta_{F}\right\rangle$ provided $\vee K$ is 1-formula, let $n(\mathscr{D})=\left\langle\bigcup_{F \in K}^{\bigcup} \tau_{F}, \underset{F \in K}{\bigwedge} \theta_{F}\right\rangle$ provided $\vee K$ is 2-formula. 
Case $5 \quad \mathrm{R}$ is $(\rightarrow \wedge)$. Dual of case 4 .

Case $6 \quad R$ is a (1)-rule, Then $\mathscr{D}$ has the form

$$
\begin{gathered}
\frac{\mathscr{l}_{1}}{\mathscr{D}_{1} \Theta, I_{i_{1}}\left(x_{1}, y_{1}\right) \cdots \Gamma \rightarrow \Theta, I_{i_{n}}\left(x_{n}, y_{n}\right)} \\
F, \Gamma \rightarrow \Theta \\
\left(\psi^{1}(\vec{x}) \in \Gamma, \quad \psi^{2}(\vec{y}) \in \Theta\right),
\end{gathered}
$$

if $n\left(\mathscr{D}_{j}\right) \neq\left\langle i_{j}, x_{j}, y_{j}\right\rangle$ for some $1 \leq j \leq n$, let $n(\mathscr{D})=n\left(\mathscr{D}_{j}\right)$,

if $n\left(\mathscr{D}_{j}\right)=\left\langle i_{j}, x_{j}, y_{j}\right\rangle$ for all $\mathrm{l} \leq j \leq n$,

let $n(\mathscr{D})=\left\langle\left\{\left\langle i_{j}, x_{j}, y_{j}\right\rangle ; 1 \leq j \leq n\right\}, \psi\left(f_{i_{1}}\left(x_{1}, y_{1}\right), \cdots, f_{i_{n}}\left(x_{n}, y_{n}\right)\right)\right\rangle$.

Case $7 \quad R$ is a (2)-rule. Then $\mathscr{D}$ has the form

$$
R \frac{I_{i_{1}}\left(x_{1}, y_{1}\right), \cdots, I_{i_{n}}\left(x_{n}, y_{n}\right), \Gamma \rightarrow \Theta}{\begin{array}{l}
\downarrow \mathscr{D}_{1} \\
F
\end{array}},
$$

if $n(\mathscr{D})=\left\langle i, x, y>\right.$, let $n(\mathscr{D})=n\left(\mathscr{D}_{1}\right)$,

if $n\left(\mathscr{D}_{1}\right)=\left\langle\tau, \theta>\right.$ let $\quad \theta=\theta\left(w^{i}\right)$, where $w^{i}=\left\langle f_{i_{1}}\left(x_{1}, y_{1}\right), \cdots, f_{i_{n}}\left(x_{n}, y_{n}\right)\right\rangle$,

$$
\tau_{1}=\tau-\left\{\left\langle i_{1}, x_{1}, y_{1}\right\rangle, \cdots,\left\langle i_{n}, x_{n}, y_{n}\right\rangle\right\} \text {, }
$$$$
\theta_{1}=(\exists \vec{v}) \theta(\vec{v})
$$

and $\quad n(\mathscr{D})=\left\langle\tau_{1}, \theta_{1}\right\rangle$.

Case $8 \quad R$ is a (3)-rule. Dual of case 7 .

Case $9 R$ is a (4)-rule. Then $\mathscr{D}$ has the form

$$
R \frac{I_{i}\left(x_{1}, y_{1}\right), \cdots, I_{i}\left(x_{n}, y_{n}\right), \Gamma \rightarrow \Theta\left(i \geq i_{0}\right)}{F, \Gamma \rightarrow \Theta}
$$

By the definition of $\Psi$-sequents, there are only finitely many $I_{i}(x, y)$ in $\Gamma$, hence there is an $i_{1} \geq i_{0}$ such that $I_{i}(x, y)$ does not belong to $\Gamma$ for every $i \geq i_{1}, x, y$ $\epsilon F V$.

Then clearly $n\left(\mathscr{D}_{i_{1}}\right) \neq\langle i, x, y\rangle$ for any $i<\omega, x, y$.

So, $n\left(\mathscr{D}_{i_{1}}\right)=\langle\tau, \theta\rangle, \theta=\theta\left(\vec{w}^{i_{1}}\right)$, where $\vec{w}^{i_{1}}=\left\langle f_{i_{1}}\left(x_{1}, y_{1}\right), \cdots f_{i_{1}}\left(x_{n}, y_{n}\right)\right\rangle$.

Let $\quad \tau_{1}=\tau-\left\{\left\langle i_{1}, x_{1}, y_{1}\right\rangle, \cdots,\left\langle i_{1}, x_{n}, y_{n}\right\rangle\right\}$ and $\theta_{1}=(\exists \vec{v}) \theta(\vec{v})$.

Then every free variable in $\theta$ belongs to $\bigcup_{i>i_{0}} V_{i}$, hence $\theta \in \Delta_{I}(\Psi)$.

Let $n(\mathscr{D})=\left\langle\boldsymbol{\tau}_{1}, \theta_{\mathbf{1}}\right\rangle$.

Case $10 \quad R$ is a (5)-rule. Dual of Case 9 .

Case $11 R$ is a (6)-rule. Then $\mathscr{D}$ has the form

$$
\begin{aligned}
& \downarrow \mathscr{D}_{1} \quad \mathscr{D}_{m} \quad \downarrow \mathscr{D}_{m+1} \\
& R \frac{\Gamma \rightarrow \Theta, I_{j_{1}}\left(x_{11}, y_{11}\right), \cdots, \Gamma \rightarrow \Theta, I_{j_{m}}\left(x_{1 m}, y_{1 m}\right) \quad I_{i_{1}}\left(x_{1}, y_{1}\right), \cdots, I_{i_{n}}\left(x_{n}, y_{n}\right), \Gamma \rightarrow \Theta}{F, \Gamma \rightarrow \Theta}
\end{aligned}
$$




$$
\begin{aligned}
& \text { if } n\left(\mathscr{D}_{p}\right) \neq\left\langle j_{p}, x_{p}, y_{p}\right\rangle \text { for some } 1 \leq p \leq m \text {, let } n(\mathscr{D})=n\left(\mathscr{D}_{p}\right), \\
& \text { if } n\left(\mathscr{D}_{\mathrm{m}+1}\right)=\langle i, x, y\rangle \text {, let } n(\mathscr{D})=\langle i, x, y\rangle, \\
& \text { if } n\left(\mathscr{D}_{\mathrm{m}+1}\right)=\langle\tau, \theta\rangle \text { and } n\left(\mathscr{D}_{p}\right)=\left\langle j_{p}, x_{p}, p_{p}\right) \text { for all } 1 \leq p \leq m \text { where } \theta=\theta\left(w^{i}\right), \\
& \qquad w^{i}=\left\langle f_{i_{1}}\left(x_{1}, y_{1}\right), \cdots, f_{i_{n}}\left(x_{n}, y_{n}\right)\right\rangle, \boldsymbol{P}=\boldsymbol{\phi}\left(w^{i}, w_{1}{ }^{j}\right), \\
& \qquad w_{1}^{j}=\left\langle f_{j_{1}}\left(x_{11}, y_{11}\right), \cdots, f_{j_{m}}\left(x_{1 m}, y_{1 m}\right)\right\rangle, \\
& \text { let } \tau_{1}=\tau^{U}\left[\left\langle j_{1}, x_{11}, y_{11}\right\rangle, \cdots,\left\langle j_{m}, x_{1 m}, y_{1 m}\right\rangle\right\}-\left\{\left\langle i_{1}, x_{1}, y_{1}\right\rangle, \cdots,\left\langle i_{n}, x_{n}, y_{n}\right\rangle\right\}, \\
& \theta_{1}=(\exists \vec{v})\left(\varphi\left(\vec{v}, w_{1}^{j}\right)_{\wedge} \theta(\vec{v})\right) \text { and } n(\mathscr{D})=\left\langle\tau_{1}, \theta_{1}\right\rangle .
\end{aligned}
$$

Case $12 R$ is a (7)-rule. Dual of case 11 .

Case $13 R$ is a (8)-rule. Then $\mathscr{D}$ has the form

$$
R \frac{I_{i_{1}}\left(x_{1}, y_{1}\right), \cdots, I_{i_{n}}\left(x_{n}, y_{n}\right), \psi^{2}(\vec{y}), \Gamma \rightarrow \Theta}{\stackrel{\mathscr{D}_{1}}{ }}
$$

$$
\begin{aligned}
& \text { if } n\left(\mathscr{D}_{1}\right)=\langle i, x, y\rangle \text {, let } n(\mathscr{D})=n\left(\mathscr{D}_{1}\right), \\
& \text { if } n\left(\mathscr{D}_{1}\right)=\left\langle\tau, \theta\left(w^{i}\right)\right\rangle \text {, where } w^{i}=\left\langle f_{i_{1}}\left(x_{1}, y_{1}\right), \cdots, f_{i_{n}}\left(x_{n}, y_{n}\right)\right\rangle \text {, let } \\
& \tau_{1}=\tau-\left\{\left\langle i_{1}, x_{1}, y_{1}\right\rangle, \cdots,\left\langle i_{n}, x_{n}, y_{n}\right\rangle\right\}, \theta_{1}=(\exists \vec{u})\left(\psi(\vec{u})_{\wedge} \theta(\vec{u})\right) \text { and } n(\mathscr{D})=\left\langle\tau_{1}, \theta_{1}\right\rangle .
\end{aligned}
$$

Case $14 \quad R$ is a (9)-rule. Dual of case 13 .

We can easily check that the above definition preserves $\left(^{*}\right)$ and $\left({ }^{* *}\right)$.

\subsection{Lemma.}

For any $I$-primitive set $\Psi$, any finite sequence $w^{i}$ of distinct free variables and any formulas $\varphi\left(w^{i}\right), \psi\left(w^{i}\right)$ in $\mathscr{L}$,

if

$$
\begin{aligned}
& \Psi \vdash_{L I}(\forall \vec{u})(\forall \vec{v})\left(I_{i}(\vec{u}, \vec{v})_{\wedge} \phi^{1}(\vec{u}) . \supset \psi^{2}(\vec{v})\right) \text {, then } \vdash_{\mathscr{L}} \boldsymbol{\varphi} \supset \theta \\
& \text { and } \vdash_{\mathscr{L}} \theta \supset \psi \text { for some } \theta \in \Delta_{I}(\Psi) \text { whose free variables are among } w^{2} .
\end{aligned}
$$

Proof Suppose $\Psi \vdash_{L I}(\forall \vec{u})(\forall \vec{v})\left(I_{i}(\vec{u}, \vec{v})_{\Lambda} \varphi^{1}(\vec{u}) . \supset \psi^{2}(\vec{v})\right)$.

Let $\vec{x}$ be a sequence of distinct free variables of the same length as $w^{i}$.

Then

$$
\Psi \underset{L^{I}}{\vdash} I_{i}(\vec{x}, \vec{x}), \phi^{1}(\vec{x}) \rightarrow \psi^{2}(\vec{x})
$$

Hence for some countable $\Psi_{1} \subseteq \Psi$

$$
\vdash_{L I} \Psi_{1}, I_{i_{1}}\left(x_{1}, x_{1}\right), \cdots, I_{i_{n}}\left(x_{n}, x_{n}\right), \varphi^{1}(\vec{x}) \rightarrow \psi^{2}(\vec{x}) .
$$

Since $\Psi_{1}, I_{i_{1}}\left(x_{1}, x_{1}\right), \cdots, I_{i_{n}}\left(x_{n}, y_{n}\right), \phi^{1}(\vec{x}) \rightarrow \psi^{2}(\vec{x})$ is a $\Psi$-sequent, there is a $\Psi$-derivation $\mathscr{D}$ of this sequent by lemma 2.7. Let $n(\mathscr{D})=\theta_{1}\left(\cdots f_{i j}\left(x_{j}, x_{j}\right) \cdots\right)$, and $\theta=\theta_{1}\left(\cdots w_{j}{ }^{i j} \cdots\right)$. Then this $\theta$ has the desired properties.

From lemma 1.2, lemma 2.5, and lemma 3.2, we have the following two theorems. 
3.3 THEOREM ( $I$-interpolation theorem on $I$-primitive sets).

Every I-primitive set is I-interpolatable.

3.4 Theorem ( $I$-characterization theorem on $I$-primitive sets).

$\Delta_{I}(\Psi)$ is an I-characterization set of $\Psi$, for each I-primitive set $\Psi$.

3.5 Remark on Feferman's interpolation theorem on many sorted logic.

We shall use Feferman's terminology in [7]. So, we assume that the reader is familiar with it.

In [7], he proved an interpolation theorem in many sorted logic and stated that it is very difficult to reduce his interpolation theorem to that in single sorted logic.

But if we use our method, we can easily reduce his interpolation theorem in many sorted logic to that of single sorted logic.

For each formula $\varphi$ in many sorted logic, $\varphi^{*}$ is a single sorted formula associated to $\varphi$ by the standard relativization method. This is, each occurrence of a quantifier $(\exists u)(\cdots)$ in $\varphi$ with $u$ of sort $j$ is replaced by $(\exists u)\left(m_{j}(u)_{\wedge} \cdots\right)$, while each occurence of $(\forall u)(\cdots)$ is replaced by $(\forall u)\left(m_{j}(u) \supset \cdots\right)$.

Then from a derivation of $\phi \supset \psi$, one gets a derivation of

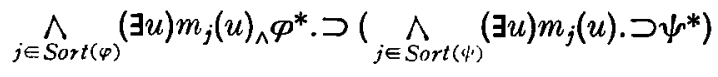

in single-sorted logic.

We shall show that from $\left(^{*}\right)$ we get an interpolating formula $\theta$, having the desired propertes, of $\varphi \supset \psi$.

Let $\Psi$ be the set of $I$-primitive sentences such that

(1) $(\forall \vec{u})(\forall \vec{v})\left(I_{0}(\vec{u}, \vec{v})_{\wedge} P^{1}(\vec{u}) . \supset P^{2}(\vec{v})\right)$, where $P \in \operatorname{Rel}^{+}(\varphi)_{\Pi} \operatorname{Rel}+(\psi)$ or $P$ is the equality symbol.

(2) $(\forall \vec{u})(\forall \vec{v})\left(I_{0}(\vec{u}, \vec{v})_{\wedge} \rightarrow P^{1}(\vec{u}) . \supset \neg P^{2}(\vec{v})\right)$, where $P \in \operatorname{Rel}^{-}(\varphi)_{n} \operatorname{Rel}^{-}(\psi)$ or $P$ is the equality symbol.

(3) $(\forall u)(\exists v)\left(m_{j}{ }^{1}(u) \supset I_{0}(u, v)_{\wedge} m_{j}{ }^{2}(v)\right)$ for $j \in E x(\psi)$.

(4) $(\forall v)(\exists u)\left(m_{j}^{2}(v) \supset I_{0}(u, v)_{\wedge} m_{j}^{2}(u)\right)$ for $j \in U n(\varphi)$.

Then $\Psi$ is $I$-primitive and

$$
\begin{gathered}
\Delta_{I}(\Psi)=\left\{\theta^{*} ; U n(\theta) \subseteq U n(\varphi), E x(\theta) \subseteq E x(\psi),\right. \\
\operatorname{Rel}^{+}(\theta) \subseteq \operatorname{Rel}^{+}(\varphi)_{\cap} \operatorname{Rel}^{+}(\psi), \\
\left.\operatorname{Rel}^{-}(\theta) \subseteq \operatorname{Rel}^{-}(\varphi)_{ก} \operatorname{Rel}^{-}(\psi)\right\} .
\end{gathered}
$$

From (*), we have

$$
\begin{gathered}
\left.\Psi \vdash_{L^{I}} \underset{j=\widehat{\operatorname{Sort}(\varphi)}}{\wedge}(\exists u) m_{j}{ }^{1}(u)_{\wedge}\left(\phi^{*}\right)^{1} \rightarrow{ }_{j \in \widehat{\operatorname{Sort}(\psi)}}(\exists u) m_{j}{ }^{2}(u) \cdot \supset\left(\Psi^{*}\right)^{2}\right) \\
-148-
\end{gathered}
$$


Hence there is a $\theta^{*} \epsilon \Delta_{I}(\Psi)$ such that

$$
\underset{j \in \operatorname{Sort}(\varphi)}{\wedge}(\exists u) m_{j}(u) \vdash \boldsymbol{\varphi}^{*} \supset \theta^{*} \text { and } \underset{j \in \operatorname{Sort}(\psi)}{\wedge}(\exists u) m_{j}(u) \vdash \theta^{*} \supset \psi^{*}
$$

Therefore $\varphi \supset \theta$ and $\theta \supset \psi$ are provable in many sorted logic and $\theta$ has the desired properties. i.e.

$$
\begin{aligned}
& \operatorname{Rel}^{+}(\theta) \subseteq \operatorname{Rel}^{+}(\varphi)_{n} \operatorname{Rel}^{+}(\psi), \\
& \operatorname{Rel}^{-}(\theta) \subseteq \operatorname{Rel}^{-}(\varphi)_{n} \operatorname{Rel}^{-}(\psi), \\
& E x(\theta) \subseteq E x(\psi), \\
& U n(\theta) \subseteq U n(\varphi) .
\end{aligned}
$$

\section{References}

[1] E.W. Beth, On Padoa's method in the theory of definition, Indag. Math., 15 (1953), 330-339

[2] W. Craig, Linear reasoning, A new form of the Herbrand-Gentzen theorem, J. Symbolic Logic, 22 (1957), 250-268

[3] W. Craig, Three uses of the Herbrand-Gentzen theorem in relating model theory and proof theory, J. Symbolic Logic, 22 (1957), 269-285

[4] C.C. Chang, On unions of chains of models, Proc. Amer. Math. Soc., 10 (1959), 120-127

[5] C.C. Chang and H.J. Keisler, Continuous model theory, Princeton U.P., 1966

[6] S. Feferman, Persistent and invarient formulas for outer extensions, Compos. Math., 20 (1968), 29-52

[7] S. Feferman, Lectures on Proof theory, Proceeding of the Summer School in Logic, Leeds, 1967, Lecture Notes in Mathematics, No. 70, Springer-Verlag, 1968, $1-107$

[8] S. Feferman and G. Kreisel, Persistent and invarient formulas relative to theories of higher order, Bull. Amer. Math. Soc., 72 (1966), 480-485

[9] H.J. Keisler, The theory of models with generalized atomic formulas, J. Symbolic Logic, 25 (1960), 1-26

[10] H.J. Keilser, Unions of relational systems, Proc. Amer. Math. Soc., 15 (1964), 540545

[11] H.J. Keisler, Reduced products and Horn classes, Trans. Amer. Math. Soc., 117 (1965), 307-328

[12] H.J. Keisler, Finite approximations of infinitely long formulas, in The theory of Models (eds. Addison, Henkin, Tarski), North-Holland, (1965), 158-169

[13] H.J. Keisler, Some applications of infinitely long formulas, J. Symbolic Logic, 30 (1965), 339-349

[14] H.J. Keisler, Model theory for $\mathrm{L}_{w_{1} w}$, preprint

[15] H.J. Keisler and M. Morley, Elementary extensions of models of set theory, Israel J. Math., 6 (1968), 49-65

[16] R.C. Lyndon, Properties preserved under algebraic constructions, Bull. Amer. Math. Soc., 65 (1959), 287-299

[17] R.C. Lyndon, An interpolation theorem in the predicate calculus, Pacific J. Math., 9 (1959), 129-142

[18] R.C. Lyndon, Properties preserved under homomorphism, Pacific J. Math., 9 (1959), 143-154

[19] J. Los, On the extending of models, I, Fund, Math., 42 (1955), 38-54

[20] E.G.K. Lopez-Escobar, An interpolation theorem for denumerably long formulas, Fund. Math., 57 (1965), 253-272 
[21] R. MacDowell and E. Specker, Modelle der Arithmetik, Infinistic Methods. Proc. 1959 Warsaw Symposium on Foundations of Mathematics, (1961), 257-263

[22] M. Makkai, On the model theory of denumerably long formulas with finite strings of quantifiers, J. Symbolic Logic, 34 (1969), 437-459

[23] J. Malitz, Universal classes in infinitary languages, Duke Math. J., 36 (1969), 621-630

[24] S. Maehara, On the interpolation theorem of Craig (Japanese), Sugaku, 12 (1960/61), 235-237

[25] N. Motohashi, On normal operations on models, J. Math. Soc. Japan, 21 (1969), 564573

[26] N. Motohashi, A theorem in the theory of definition, J. Math. Soc. Japan, 22 (1970), 490-494

[27] N. Motohashi, Object logic and morphism logic, to appear.

[28] M. Morley, Omitting classes of elements, in The theory of models (eds, Addison, Henkin, Tarski), North-Holland, (1965), 265-273

[29] M. Morley and R. Vaught, Homogeneous universal models, Math. Scand., 11 (1962), 37-57

[30] B.F. Nebres, A syntactic characterization of infinitary sentences preserved under unions of models, Notices Amer. Math, Soc., 16 (1969), 423-424

[31] A. Robinson, Introduction to model theory, North-Holland, 1963

[32] A. Robinson, Obstructions to arithmetical extension and the theorem of Los and Susko, Indag. Math., 21 (1959), 489-495

[33] J. Shoenfield, Mathematical Logic, Addison-Wesley, 1967

[34] A. Tarski, Contributions to the theory of models, Indag. Math., 16 (1954), Part I, 572-581, Part II, 582-588

[35] B. Weglorz, Some preservation theorems, Colloq. Math., 17 (1967), 262-276

[36] T. Nagashima, An extension of the Craig-Schütte interpolation theorem, Annals of the Japan Association for Philosophy of Science, 3 (1966), 12-18 